\title{
Entanglement Entropy of Black Holes
}

\author{
Sergey N. Solodukhin \\ Laboratoire de Mathématiques et Physique Théorique \\ Université François-Rabelais Tours Fédération Denis Poisson - CNRS, \\ Parc de Grandmont, 37200 Tours, France \\ email: Sergey.Solodukhin@lmpt.univ-tours.fr
}

Accepted on 23 August 2011

Published on 21 October 2011

\begin{abstract}
The entanglement entropy is a fundamental quantity, which characterizes the correlations between sub-systems in a larger quantum-mechanical system. For two sub-systems separated by a surface the entanglement entropy is proportional to the area of the surface and depends on the UV cutoff, which regulates the short-distance correlations. The geometrical nature of entanglement-entropy calculation is particularly intriguing when applied to black holes when the entangling surface is the black-hole horizon. I review a variety of aspects of this calculation: the useful mathematical tools such as the geometry of spaces with conical singularities and the heat kernel method, the UV divergences in the entropy and their renormalization, the logarithmic terms in the entanglement entropy in four and six dimensions and their relation to the conformal anomalies. The focus in the review is on the systematic use of the conical singularity method. The relations to other known approaches such as 't Hooft's brick-wall model and the Euclidean path integral in the optical metric are discussed in detail. The puzzling behavior of the entanglement entropy due to fields, which non-minimally couple to gravity, is emphasized. The holographic description of the entanglement entropy of the blackhole horizon is illustrated on the two- and four-dimensional examples. Finally, I examine the possibility to interpret the Bekenstein-Hawking entropy entirely as the entanglement entropy.
\end{abstract}

This review is licensed under a Creative Commons Attribution-Non-Commercial-NoDerivs 3.0 Germany License. http://creativecommons.org/licenses/by-nc-nd/3.0/de/ 


\section{Imprint / Terms of Use}

Living Reviews in Relativity is a peer reviewed open access journal published by the Max Planck Institute for Gravitational Physics, Am Mühlenberg 1, 14476 Potsdam, Germany. ISSN 1433-8351.

This review is licensed under a Creative Commons Attribution-Non-Commercial-NoDerivs 3.0

Germany License: http://creativecommons.org/licenses/by-nc-nd/3.0/de/

Because a Living Reviews article can evolve over time, we recommend to cite the article as follows:

Sergey N. Solodukhin, "Entanglement Entropy of Black Holes",

Living Rev. Relativity, 14, (2011), 8. [Online Article]: cited [<date $>$ ], http://www.livingreviews.org/lrr-2011-8

The date given as $<$ date $>$ then uniquely identifies the version of the article you are referring to.

\section{Article Revisions}

Living Reviews supports two ways of keeping its articles up-to-date:

Fast-track revision A fast-track revision provides the author with the opportunity to add short notices of current research results, trends and developments, or important publications to the article. A fast-track revision is refereed by the responsible subject editor. If an article has undergone a fast-track revision, a summary of changes will be listed here.

Major update A major update will include substantial changes and additions and is subject to full external refereeing. It is published with a new publication number.

For detailed documentation of an article's evolution, please refer to the history document of the article's online version at http://www. livingreviews.org/lrr-2011-8. 


\section{Contents}

1 Introduction $\quad 5$

2 Entanglement Entropy in Minkowski Spacetime $\quad 7$

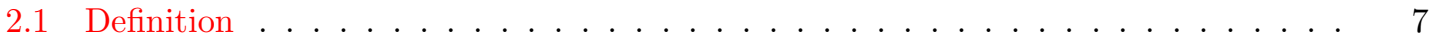

2.2 Short-distance correlations . . . . . . . . . . . . . . . . . . . . 7

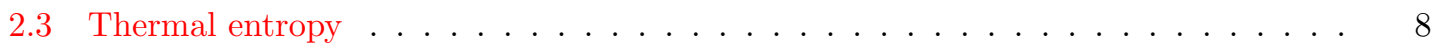

2.4 Entropy of a system of finite size at finite temperature . . . . . . . . . . . . 8

2.5 Entropy in $(1+1)$-dimensional spacetime . . . . . . . . . . . . . . 8

2.6 The Euclidean path integral representation and the replica method . . . . . . . . . 9

2.7 Uniqueness of analytic continuation . . . . . . . . . . . . . . . . . . . . . 10

2.8 Heat kernel and the Sommerfeld formula . . . . . . . . . . . . . . . . . . . . 11

2.9 An explicit calculation . . . . . . . . . . . . . . . . . . . . . . . 12

2.10 Entropy of massive fields . . . . . . . . . . . . . . . . . . . . . 13

2.11 An expression in terms of the determinant of the Laplacian on the surface . . . . . 13

2.12 Entropy in theories with a modified propagator . . . . . . . . . . . . . . . . 14

2.13 Entanglement entropy in non-Lorentz invariant theories . . . . . . . . . . . . . 15

2.14 Arbitrary surface in curved spacetime: general structure of UV divergences . . . . 16

3 Entanglement Entropy of Non-Degenerate Killing Horizons 18

3.1 The geometric setting of black-hole spacetimes . . . . . . . . . . . . . 18

3.2 Extrinsic curvature of horizon, horizon as a minimal surface . . . . . . . . . . . . . 19

3.3 The wave function of a black hole . . . . . . . . . . . . . . . . . . . . 19

3.4 Reduced density matrix and entropy . . . . . . . . . . . . . . . . . . . . . . 19

3.5 The role of the rotational symmetry . . . . . . . . . . . . . . . . . 20

3.6 Thermality of the reduced density matrix of a Killing horizon . . . . . . . . . . . 20

3.7 Useful mathematical tools . . . . . . . . . . . . . . . . . . . . . . . . 21

3.7.1 Curvature of space with a conical singularity . . . . . . . . . . . . 21

3.7.2 The heat kernel expansion on a space with a conical singularity . . . . . . 23

3.8 General formula for entropy in the replica method, relation to the Wald entropy . 24

3.9 UV divergences of entanglement entropy for a scalar field . . . . . . . . . . . . 25

3.9.1 The Reissner-Nordström black hole . . . . . . . . . . . . . . . 26

3.9.2 The dilatonic charged black hole . . . . . . . . . . . . . . . 27

3.10 Entanglement Entropy of the Kerr-Newman black hole . . . . . . . . . . . . . . 28

3.10 .1 Euclidean geometry of Kerr-Newman black hole . . . . . . . . . . . . . 28

3.10 .2 Extrinsic curvature of the horizon . . . . . . . . . . . . . . . . . . . . . . . . . .

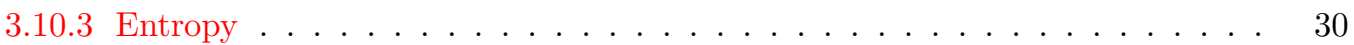

3.11 Entanglement entropy as one-loop quantum correction . . . . . . . . . . . . . . . . . 31

3.12 The statement on the renormalization of the entropy . . . . . . . . . . . . . . 31

3.13 Renormalization in theories with a modified propagator . . . . . . . . . . . . 33

3.14 Area law: generalization to higher spin fields . . . . . . . . . . . . . . . . . . 34

3.15 Renormalization of entropy due to fields of different spin . . . . . . . . . . . . . . . 35

3.16 The puzzle of non-minimal coupling . . . . . . . . . . . . . . . . . . . . . . . . . . . . . . . . . . . .

3.17 Comments on the entropy of interacting fields . . . . . . . . . . . . . . . 38

4 Other Related Methods $\quad 40$

4.1 Euclidean path integral and thermodynamic entropy . . . . . . . . . . . . . 40

4.2 't Hooft's brick-wall model . . . . . . . . . . . . . . . . . . . . . . . . . . . . . . . . . . . . . . . . . . . . . . .

4.2.1 WKB approximation, Pauli-Villars fields . . . . . . . . . . . . . 42

4.2.2 Euclidean path integral approach in terms of optical metric . . . . . . . 45 
5 Some Particular Cases $\quad 50$

5.1 Entropy of a 2D black hole . . . . . . . . . . . . . . . . . . 50

5.2 Entropy of 3D Banados-Teitelboim-Zanelli (BTZ) black hole . . . . . . . . . . . 52

5.2 .1 BTZ black-hole geometry . . . . . . . . . . . . . . . 52

5.2 .2 Heat kernel on regular BTZ geometry . . . . . . . . . . . . . . . . 53

5.2 .3 Heat kernel on conical BTZ geometry . . . . . . . . . . . . . . . 53

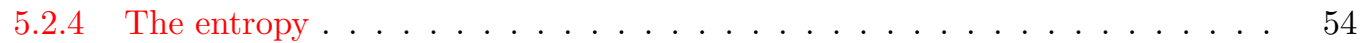

5.3 Entropy of d-dimensional extreme black holes . . . . . . . . . . . . . . . . . . 55

5.3 .1 Universal extremal limit . . . . . . . . . . . . . . . . . . . 56

5.3.2 Entanglement entropy in the extremal limit . . . . . . . . . . . . . 57

6 Logarithmic Term in the Entropy of Generic Conformal Field Theory 61

6.1 Logarithmic terms in 4-dimensional conformal field theory . . . . . . . . . . . . . . 62

6.2 Logarithmic terms in 6-dimensional conformal field theory . . . . . . . . . . . . . . 64

6.3 Why might logarithmic terms in the entropy be interesting? . . . . . . . . . . . 66

7 A Holographic Description of the Entanglement Entropy of Black Holes $\quad 68$

7.1 Holographic proposal for entanglement entropy . . . . . . . . . . . . . . . 68

7.2 Proposals for the holographic entanglement entropy of black holes . . . . . . . . . 69

7.3 The holographic entanglement entropy of $2 \mathrm{D}$ black holes . . . . . . . . . . . . . 70

7.4 Holographic entanglement entropy of higher dimensional black holes . . . . . . . . 71

8 Can Entanglement Entropy Explain the Bekenstein-Hawking Entropy of Black Holes?

8.1 Problems of interpretation of the Bekenstein-Hawking entropy as entanglement en-

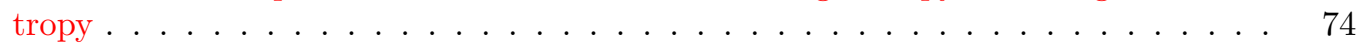

8.2 Entanglement entropy in induced gravity . . . . . . . . . . . . . . . . . . . . . . . . . . . . . . . . . . .

8.3 Entropy in brane-world scenario . . . . . . . . . . . . . . . . . . . . . 75

8.4 Gravity cut-off . . . . . . . . . . . . . . . . . . . . . . . . . . 76

8.5 Kaluza-Klein example . . . . . . . . . . . . . . . . . . . . . 77

9 Other Directions of Research $\quad \mathbf{7 8}$

9.1 Entanglement entropy in string theory . . . . . . . . . . . . . . . . . 78

9.2 Entanglement entropy in loop quantum gravity . . . . . . . . . . . . . . . . 79

9.3 Entropy in non-commutative theories and in models with minimal length . . . . . 79

9.4 Transplanckian physics and entanglement entropy . . . . . . . . . . . . . . 80

9.5 Entropy of more general states . . . . . . . . . . . . . . . . . . . . . . . . 80

9.6 Non-unitary time evolution . . . . . . . . . . . . . . . . . . . . 80

10 Concluding remarks $\quad 81$

11 Acknowledgments $\quad 81$

$\begin{array}{lr}\text { References } & 82\end{array}$

\section{List of Tables}

1 Coefficients of the logarithmic term in the entanglement entropy of an extreme Reissner-Nordström black hole. . . . . . . . . . . . . . . . . . . 60 


\section{Introduction}

One of the mysteries in modern physics is why black holes have an entropy. This entropy, known as the Bekenstein-Hawking entropy, was first introduced by Bekenstein [18, 19, 20] as a rather useful analogy. Soon after that, this idea was put on a firm ground by Hawking [128] who showed that black holes thermally radiate and calculated the black-hole temperature. The main feature of the Bekenstein-Hawking entropy is its proportionality to the area of the black-hole horizon. This property makes it rather different from the usual entropy, for example the entropy of a thermal gas in a box, which is proportional to the volume. In 1986 Bombelli, Koul, Lee and Sorkin [23] published a paper in which they considered the reduced density matrix, obtained by tracing over the degrees of freedom of a quantum field that are inside the horizon. This procedure appears to be very natural for black holes, since the black hole horizon plays the role of a causal boundary, which does not allow anyone outside the black hole to have access to the events, which take place inside the horizon. Another attempt to understand the entropy of black holes was made by 't Hooft in 1985 [214]. His idea was to calculate the entropy of the thermal gas of Hawking particles, which propagate just outside the horizon. This calculation has uncovered two remarkable features: the entropy does turn out to be proportional to the horizon area, however, in order to regularize the density of states very close to the horizon, it was necessary to introduce the brick wall, a boundary, which is placed at a small distance from the actual horizon. This small distance plays the role of a regulator in the 't Hooft's calculation. Thus, the first indications that entropy may grow as area were found.

An important step in the development of these ideas was made in 1993 when a paper of Srednicki [208] appeared. In this very inspiring paper Srednicki calculated the reduced density and the corresponding entropy directly in flat spacetime by tracing over the degrees of freedom residing inside an imaginary surface. The entropy defined in this calculation has became known as the entanglement entropy. Sometimes the term geometric entropy is used as well. The entanglement entropy, as was shown by Srednicki, is proportional to the area of the entangling surface. This fact is naturally explained by observing that the entanglement entropy is non-vanishing due to the short-distance correlations present in the system. Thus, only modes, which are located in a small region close to the surface, contribute to the entropy. By virtue of this fact, one finds that the size of this region plays the role of the UV regulator so that the entanglement entropy is a UV sensitive quantity. A surprising feature of Srednicki's calculation is that no black hole is actually needed: the entanglement entropy of a quantum field in flat spacetime already establishes the area law. In an independent paper, Frolov and Novikov [99] applied a similar approach directly to a black hole. These results have sparked interest in the entanglement entropy. In particular, it was realized that the brick-wall model of 't Hooft studies a similar entropy and that the two entropies are in fact related. On the technical side of the problem, a very efficient method was developed to calculate the entanglement entropy. This method, first considered by Susskind [211], is based on a simple replica trick, in which one first introduces a small conical singularity at the entangling surface, evaluates the effective action of a quantum field on the background of the metric with a conical singularity and then differentiates the action with respect to the deficit angle. By means of this method one has developed a systematic calculation of the UV divergent terms in the geometric entropy of black holes, revealing the covariant structure of the divergences [33, 197, 111]. In particular, the logarithmic UV divergent terms in the entropy were found [196]. The other aspect, which was widely discussed in the literature, is whether the UV divergence in the entanglement entropy could be properly renormalized. It was suggested by Susskind and Uglum [213] that the standard renormalization of Newton's constant makes the entropy finite, provided one considers the entanglement entropy as a quantum contribution to the Bekenstein-Hawking entropy. However, this proposal did not answer the question of whether the Bekenstein-Hawking entropy itself can be considered as an entropy of entanglement. It was proposed by Jacobson [141] that, in models 
in which Newton's constant is induced in the spirit of Sakharov's ideas, the Bekenstein-Hawking entropy would also be properly induced. A concrete model to test this idea was considered in [97].

Unfortunately, in the 1990s, the study of entanglement entropy could not compete with the booming success of the string theory (based on D-branes) calculations of black-hole entropy [209]. The second wave of interest in entanglement entropy started in 2003 with work studying the entropy in condensed matter systems and in lattice models. These studies revealed the universality of the approach based on the replica trick and the efficiency of the conformal symmetry to compute the entropy in two dimensions. Black holes again came into the focus of study in 2006 after work of Ryu and Takayanagi [189] where a holographic interpretation of the entanglement entropy was proposed. In this proposal, in the frame of the AdS/CFT correspondence, the entanglement entropy, defined on a boundary of anti-de Sitter, is related to the area of a certain minimal surface in the bulk of the anti-de Sitter spacetime. This proposal opened interesting possibilities for computing, in a purely geometrical way, the entropy and for addressing in a new setting the question of the statistical interpretation of the Bekenstein-Hawking entropy.

The progress made in recent years and the intensity of the on-going research indicate that entanglement entropy is a very promising direction, which, in the coming years, may lead to a breakthrough in our understanding of black holes and quantum gravity. A number of very nice reviews appeared in recent years that address the role of entanglement entropy for black holes [21, 90, 146, 54]; review the calculation of entanglement entropy in quantum field theory in flat spacetime [81, 37] and the role of the conformal symmetry [31]; and focus on the holographic aspects of the entanglement entropy [185, 11]. In the present review I build on these works and focus on the study of entanglement entropy as applied to black holes. The goal of this review is to collect a complete variety of results and present them in a systematic and self-consistent way without neglecting either technical or principal aspects of the problem. 


\section{Entanglement Entropy in Minkowski Spacetime}

\subsection{Definition}

Consider a pure vacuum state $\mid \psi>$ of a quantum system defined inside a space-like region $\mathcal{O}$ and suppose that the degrees of freedom in the system can be considered as located inside certain sub-regions of $\mathcal{O}$. A simple example of this sort is a system of coupled oscillators placed in the sites of a space-like lattice. Then, for an arbitrary imaginary surface $\Sigma$, which separates the region $\mathcal{O}$ into two complementary sub-regions $A$ and $B$, the system in question can be represented as a union of two sub-systems. The wave function of the global system is given by a linear combination of the product of quantum states of each sub-system, $\left|\psi>=\sum_{i, a} \psi_{i a}\right| A>_{i} \mid B>_{a}$. The states $\mid A>_{i}$ are formed by the degrees of freedom localized in the region $A$, while the states $\mid B>_{a}$ are formed by those, which are defined in region $B$. The density matrix that corresponds to a pure quantum state $|\psi\rangle$

$$
\rho_{0}(A, B)=|\psi><\psi|
$$

has zero entropy. By tracing over the degrees of freedom in region $A$ we obtain a density matrix

$$
\rho_{B}=\operatorname{Tr}_{A} \rho_{0}(A, B),
$$

with elements $\left(\rho_{B}\right)_{a b}=\left(\psi \psi^{\dagger}\right)_{a b}$. The statistical entropy, defined for this density matrix by the standard formula

$$
S_{B}=-\operatorname{Tr} \rho_{B} \ln \rho_{B}
$$

is by definition the entanglement entropy associated with the surface $\Sigma$. We could have traced over the degrees of freedom located in region $B$ and formed the density matrix $\left(\rho_{A}\right)_{i j}=\left(\psi^{T} \psi^{*}\right)_{i j}$. It is clear that ${ }^{1}$

$$
\operatorname{Tr} \rho_{A}^{k}=\operatorname{Tr} \rho_{B}^{k}
$$

for any integer $k$. Thus, we conclude that the entropy (3) is the same for both density matrices $\rho_{A}$ and $\rho_{B}$,

$$
S_{A}=S_{B}
$$

This property indicates that the entanglement entropy for a system in a pure quantum state is not an extensive quantity. In particular, it does not depend on the size of each region $A$ or $B$ and thus is only determined by the geometry of $\Sigma$.

\subsection{Short-distance correlations}

On the other hand, if the entropy (3) is non-vanishing, this shows that in the global system there exist correlations across the surface $\Sigma$ between modes, which reside on different sides of the surface. In this review we shall consider the case in which the system in question is a quantum field. The short-distance correlations that exist in this system have two important consequences:

- the entanglement entropy becomes dependent on the UV cut-off $\epsilon$, which regularizes the short-distance (or the large-momentum) behavior of the field system

- to leading order in $\epsilon^{-1}$ the entanglement entropy is proportional to the area of the surface $\Sigma$

\footnotetext{
${ }^{1}$ For finite matrices this property indicates that the two density matrices have the same eigenvalues.
} 
For a free massless scalar field the 2-point correlation function in $d$ spacetime dimensions has the standard form

$$
<\phi(x), \phi(y)>=\frac{\Omega_{d}}{|x-y|^{d-2}},
$$

where $\Omega_{d}=\frac{\Gamma\left(\frac{d-2}{2}\right)}{4 \pi^{d / 2}}$. Correspondingly, the typical behavior of the entanglement entropy in $d$ dimensions is

$$
S \sim \frac{A(\Sigma)}{\epsilon^{d-2}}
$$

where the exact pre-factor depends on the regularization scheme. Although the similarity between (5) and (6) illustrates well the field-theoretical origin of the entanglement entropy, the exact relation between the short-distance behavior of 2-point correlation functions in the field theory and the UV divergence of the entropy is more subtle, as we shall discuss later in the paper.

\subsection{Thermal entropy}

Instead of a pure state one could have started with a mixed thermal state at temperature $T$ with density matrix $\rho_{0}(A, B)=e^{-T^{-1} H(A, B)}$, where $H(A, B)$ is the Hamiltonian of the global system. In this case the relation (4) is no more valid and the entropy depends on the size of the total system as well as on the size of each sub-system. By rather general arguments, in the limit of large volume the reduced density matrix approaches the thermal density matrix. So that in this limit the entanglement entropy (3) reproduces the thermal entropy. For further references we give here the expression

$$
S_{\text {thermal }}=\frac{d}{\pi^{d / 2}} \Gamma\left(\frac{d}{2}\right) \zeta(d) T^{d-1} V_{d-1}
$$

for the thermal entropy of a massless field residing inside a spatial $(d-1)$-volume $V_{d-1}$ at temperature $T$.

\subsection{Entropy of a system of finite size at finite temperature}

In a more general situation one starts with a system of finite size $L$ in a mixed thermal state at temperature $T$. This system is divided by the entangling surface $\Sigma$ in two sub-systems of characteristic size $l$. Then, the entanglement entropy is a function of several parameters (if the field in question is massive then mass $m$ should be added to the parameters on which the entropy should depend)

$$
S=S(T, L, l, \epsilon)
$$

where $\epsilon$ is a UV cut-off. Clearly, the entanglement entropy in this general case is due to a combination of different factors: the entanglement between two sub-systems and the thermal nature of the initial mixed state. In $d$ dimensions even for simple geometries this function of 4 variables is not known explicitly. However, in two spacetime dimensions, in some particular cases, the explicit form of this function is known.

\subsection{Entropy in $(1+1)$-dimensional spacetime}

The state of a quantum field in two dimensions is defined on a union of intersecting intervals $A \cup B$. The 2-point correlation functions behave logarithmically in the limit of coincident points. 
Correspondingly, the leading UV divergence of the entanglement entropy in two dimensions is logarithmic. For example, for a 2D massless conformal field theory, characterized by a central charge $c$, the entropy is [208, 33, 133]

$$
S_{2 d}=\frac{c n}{6} \ln \frac{l_{A}}{\epsilon}+s\left(l_{A} / l_{B}\right)
$$

where $n$ is the number of intersections of intervals $A$ and $B$, where the sub-systems are defined, $l_{A}\left(l_{B}\right)$ is the length of the interval $A(B)$. The second term in Eq. 9) is a UV finite term. In some cases the conformal symmetry in two dimensions can be used to calculate not only the UV divergent term in the entanglement entropy but also the UV finite term, thus obtaining the complete answer for the entropy, as was shown by Holzhey, Larsen and Wilczek [133] (see [161, 29] for more recent developments). There are two different limiting cases when the conformal symmetry is helpful. In the first case, one considers a pure state of the conformal field theory on a circle of circumference $L$, the subsystem is defined on a segment of size $l$ of the circle. In the second situation, the system is defined on an infinite line, the subsystem lives on an interval of length $l$ of the line and the global system is in a thermal mixed state with temperature $T$. In Euclidean signature both geometries represent a cylinder. For a thermal state the compact direction on the cylinder corresponds to Euclidean time $\tau$ compactified to form a circle of circumference $\beta=1 / T$. In both cases the cylinder can be further conformally mapped to a plane. The invariance of the entanglement entropy under conformal transformation can be used to obtain

$$
S=\frac{c}{3} \ln \left(\frac{L}{\pi \epsilon} \sin \left(\frac{\pi l}{L}\right)\right.
$$

in the case of a pure state on a circle and

$$
S=\frac{c}{3} \ln \left(\frac{\beta}{\pi \epsilon} \sinh \left(\frac{\pi l}{\beta}\right)\right.
$$

for a thermal mixed state on an infinite line. In the limit of large $l$ the entropy (11) approaches

$$
S=\frac{c}{3} \pi l T+\frac{c}{3} \ln \left(\frac{l}{\pi \epsilon}\right)+\frac{c}{3} \ln \frac{\beta}{l}
$$

where the first term represents the entropy of the thermal gas (7) in a cavity of size $l$, while the second term represent the purely entanglement contribution (note that the intersection of $A$ and $B$ contains two points in this case so that $n=2$ ). The third term is an intermediate term due to the interaction of both factors, thermality and entanglement. This example clearly shows that for a generic thermal state the entanglement entropy is due to the combination of two factors: the entanglement between two subsystems and the thermal nature of the mixed state of the global system.

\subsection{The Euclidean path integral representation and the replica method}

A technical method very useful for the calculation of the entanglement entropy in a field theory is the the replica trick, see [33]. Here we illustrate this method for a field theory described by a secondorder Laplace-type operator. One considers a quantum field $\psi(X)$ in a $d$-dimensional spacetime and chooses the Cartesian coordinates $X^{\mu}=\left(\tau, x, z^{i}, i=1, . ., d-2\right)$, where $\tau$ is Euclidean time, such that the surface $\Sigma$ is defined by the condition $x=0$ and $\left(z^{i}, i=1, . ., d-2\right)$ are the coordinates on $\Sigma$. In the subspace $(\tau, x)$ it will be convenient to choose the polar coordinate system $\tau=r \sin (\phi)$ and $x=r \cos (\phi)$, where the angular coordinate $\phi$ varies between 0 and $2 \pi$. We note that if the field 
theory in question is relativistic, then the field operator is invariant under the shifts $\phi \rightarrow \phi+w$, where $w$ is an arbitrary constant.

One first defines the vacuum state of the quantum field in question by the path integral over a half of the total Euclidean spacetime defined as $\tau \leq 0$ such that the quantum field satisfies the fixed boundary condition $\psi(\tau=0, x, z)=\psi_{0}(x, z)$ on the boundary of the half-space,

$$
\Psi\left[\psi_{0}(x, z)\right]=\int_{\left.\psi(X)\right|_{\tau=0}=\psi_{0}(x, z)} \mathcal{D} \psi e^{-W[\psi]},
$$

where $W[\psi]$ is the action of the field. The surface $\Sigma$ in our case is a plane and the Cartesian coordinate $x$ is orthogonal to $\Sigma$. The co-dimension 2 surface $\Sigma$ defined by the conditions $x=0$ and $\tau=0$ naturally separates the hypersurface $\tau=0$ into two parts: $x<0$ and $x>0$. These are the two sub-regions $A$ and $B$ discussed in Section 2.1.

The boundary data $\psi(x, z)$ is also separated into $\psi_{-}(x, z)=\psi_{0}(x, z), x<0$ and $\psi_{+}=$ $\psi_{0}(x, z), x>0$. By tracing over $\psi_{-}(x, z)$ one defines a reduced density matrix

$$
\rho\left(\psi_{+}^{1}, \psi_{+}^{2}\right)=\int \mathcal{D} \psi_{-} \Psi\left(\psi_{+}^{1}, \psi_{-}\right) \Psi\left(\psi_{+}^{2}, \psi_{-}\right),
$$

where the path integral goes over fields defined on the whole Euclidean spacetime except a cut $(\tau=0, x>0)$. In the path integral the field $\psi(X)$ takes the boundary value $\psi_{+}^{2}$ above the cut and $\psi_{+}^{1}$ below the cut. The trace of the $n$-th power of the density matrix (14) is then given by the Euclidean path integral over fields defined on an $n$-sheeted covering of the cut spacetime. In the polar coordinates $(r, \phi)$ the cut corresponds to values $\phi=2 \pi k, k=1,2, \ldots, n$. When one passes across the cut from one sheet to another, the fields are glued analytically. Geometrically this $n$-fold space is a flat cone $C_{n}$ with angle deficit $2 \pi(1-n)$ at the surface $\Sigma$. Thus, we have

$$
\operatorname{Tr} \rho^{n}=Z\left[C_{n}\right]
$$

where $Z\left[C_{n}\right]$ is the Euclidean path integral over the $n$-fold cover of the Euclidean space, i.e., over the cone $C_{n}$. Assuming that in Eq. (15) one can analytically continue to non-integer values of $n$, one observes that

$$
-\operatorname{Tr} \hat{\rho} \ln \hat{\rho}=-\left.\left(\alpha \partial_{\alpha}-1\right) \ln \operatorname{Tr} \rho^{\alpha}\right|_{\alpha=1},
$$

where $\hat{\rho}=\rho / \operatorname{Tr} \rho$ is the renormalized matrix density. Introduce the effective action $W(\alpha)=$ $-\ln Z(\alpha)$, where $Z(\alpha)=Z\left[C_{\alpha}\right]$ is the partition function of the field system in question on a Euclidean space with conical singularity at the surface $\Sigma$. In the polar coordinates $(r, \phi)$ the conical space $C_{\alpha}$ is defined by making the coordinate $\phi$ periodic with period $2 \pi \alpha$, where $(1-\alpha)$ is very small. The invariance under the abelian isometry $\phi \rightarrow \phi+w$ helps to construct without any problem the correlation functions with the required periodicity $2 \pi \alpha$ starting from the $2 \pi$-periodic correlation functions. The analytic continuation of $\operatorname{Tr} \rho^{\alpha}$ to $\alpha$ different from 1 in the relativistic case is naturally provided by the path integral $Z(\alpha)$ over the conical space $C_{\alpha}$. The entropy is then calculated by the replica trick

$$
S=\left.\left(\alpha \partial_{\alpha}-1\right) W(\alpha)\right|_{\alpha=1}
$$

One of the advantages of this method is that we do not need to care about the normalization of the reduced density matrix and can deal with a matrix, which is not properly normalized.

\subsection{Uniqueness of analytic continuation}

The uniqueness of the analytic continuation of $\operatorname{Tr} \rho^{n}$ to non-integer $n$ may not seem obvious, especially if the field system in question is not relativistic so that there is no isometry in the polar

Living Reviews in Relativity

http: //www. livingreviews.org/lrr-2011-8 
angle $\phi$, which would allow us, without any trouble, to glue together pieces of the Euclidean space to form a path integral over a conical space $C_{\alpha}$. However, some arguments can be given that the analytic continuation to non-integer $n$ is in fact unique.

Consider a renormalized density matrix $\hat{\rho}=\frac{\rho}{\operatorname{Tr} \rho}$. The eigenvalues of $\hat{\rho}$ lie in the interval $0<\lambda<1$. If this matrix were a finite matrix we could use the triangle inequality to show that

$$
\left|\operatorname{Tr} \hat{\rho}^{\alpha}\right|<\left|(\operatorname{Tr} \hat{\rho})^{\alpha}\right|=1 \text { if } \operatorname{Re}(\alpha)>1 .
$$

For infinite-size matrices the trace is usually infinite so that a regularization is needed. Suppose that $\epsilon$ is the regularization parameter and $\operatorname{Tr}_{\epsilon}$ is the regularized trace. Then

$$
\left|\operatorname{Tr}_{\epsilon} \hat{\rho}^{\alpha}\right|<1 \text { if } \operatorname{Re}(\alpha)>1
$$

Thus $\operatorname{Tr} \hat{\rho}^{\alpha}$ is a bounded function in the complex half-plane, $\operatorname{Re}(\alpha)>1$. Now suppose that we know that $\left.\operatorname{Tr}_{\epsilon} \rho^{\alpha}\right|_{\alpha=n}=Z_{0}(n)$ for integer values of $\alpha=n, n=1,2,3, \ldots$ Then, in the region $\operatorname{Re}(\alpha)>1$, we can represent $Z(\alpha)=\operatorname{Tr}_{\epsilon} \rho^{\alpha}$ in the form

$$
Z(\alpha)=Z_{0}(\alpha)+\sin (\pi \alpha) g(\alpha)
$$

where the function $g(\alpha)$ is analytic (for $\operatorname{Re}(\alpha)>1$ ). Since by condition (17) the function $Z(\alpha)$ is bounded, we obtain that, in order to compensate for the growth of the sine in Eq. (18) for complex values of $\alpha$, the function $g(\alpha)$ should satisfy the condition

$$
|g(\alpha=x+i y)|<e^{-\pi|y|} .
$$

By Carlson's theorem [36] an analytic function, which is bounded in the region $\operatorname{Re}(\alpha)>1$ and which satisfies condition (19), vanishes identically. Thus, we conclude that $g(\alpha) \equiv 0$ and there is only one analytic continuation to non-integer $n$, namely the one given by function $Z_{0}(\alpha)$.

\subsection{Heat kernel and the Sommerfeld formula}

Consider for concreteness a quantum bosonic field described by a field operator $\mathcal{D}$ so that the partition function is $Z=\operatorname{det}^{-1 / 2} \mathcal{D}$. Then, the effective action defined as

$$
W=-\frac{1}{2} \int_{\epsilon^{2}}^{\infty} \frac{d s}{s} \operatorname{Tr} K(s)
$$

where parameter $\epsilon$ is a UV cutoff, is expressed in terms of the trace of the heat kernel $K\left(s, X, X^{\prime}\right)=<$ $X\left|e^{-s \mathcal{D}}\right| X^{\prime}>$. The latter is defined as a solution to the heat equation

$$
\left\{\begin{array}{l}
\left(\partial_{s}+\mathcal{D}\right) K\left(s, X, X^{\prime}\right)=0 \\
K\left(s=0, X, X^{\prime}\right)=\delta\left(X, X^{\prime}\right)
\end{array}\right.
$$

In order to calculate the effective action $W(\alpha)$ we use the heat kernel method. In the context of manifolds with conical singularities this method was developed in great detail in [69, 101]. In the Lorentz invariant case the invariance under the abelian symmetry $\phi \rightarrow \phi+w$ plays an important role. The heat kernel $K\left(s, \phi, \phi^{\prime}\right)$ (where we omit the coordinates other than the angle $\phi$ ) on regular flat space then depends on the difference $\left(\phi-\phi^{\prime}\right)$. This function is $2 \pi$ periodic with respect to $\left(\phi-\phi^{\prime}\right)$. The heat kernel $K_{\alpha}\left(s, \phi, \phi^{\prime}\right)$ on a space with a conical singularity is supposed to be $2 \pi \alpha$ periodic. It is constructed from the $2 \pi$ periodic quantity by applying the Sommerfeld formula [207]

$$
K_{\alpha}\left(s, \phi, \phi^{\prime}\right)=K\left(s, \phi-\phi^{\prime}\right)+\frac{i}{4 \pi \alpha} \int_{\Gamma} \cot \frac{w}{2 \alpha} K\left(s, \phi-\phi^{\prime}+w\right) d w .
$$


That this quantity still satisfies the heat kernel equation is a consequence of the invariance under the abelian isometry $\phi \rightarrow \phi+w$. The contour $\Gamma$ consists of two vertical lines, going from $(-\pi+i \infty)$ to $(-\pi-i \infty)$ and from $(\pi-i \infty)$ to $(\pi-+i \infty)$ and intersecting the real axis between the poles of the $\cot \frac{w}{2 \alpha}:-2 \pi \alpha, 0$ and $0,+2 \pi \alpha$, respectively. For $\alpha=1$ the integrand in Eq. (22) is a $2 \pi$-periodic function and the contributions of these two vertical lines cancel each other. Thus, for a small angle deficit the contribution of the integral in Eq. (22) is proportional to $(1-\alpha)$.

\subsection{An explicit calculation}

Consider an infinite $(d-2)$-plane in $d$-dimensional spacetime. The calculation of the entanglement entropy for this plane can be done explicitly by means of the heat kernel method. In flat spacetime, if the operator $\mathcal{D}$ is the Laplace operator,

$$
\mathcal{D}=-\nabla^{2}
$$

one can use the Fourier transform in order to solve the heat equation. In $d$ spacetime dimensions one has

$$
K\left(s, X, X^{\prime}\right)=\frac{1}{(2 \pi)^{d}} \int d^{d} p e^{i p_{\mu}\left(X^{\mu}-X^{\prime \mu}\right)} e^{-s F\left(p^{2}\right)} .
$$

Putting $z^{i}=z^{i}, i=1, . ., d-2$ and choosing in the polar coordinate system $(r, \phi)$, that $\phi=\phi^{\prime}+w$ we have that $p_{\mu}\left(X-X^{\prime}\right)^{\mu}=2 p r \sin \frac{w}{2} \cos \theta$, where $p^{2}=p^{\mu} p_{\mu}$ and $\theta$ is the angle between the $d$ vectors $p^{\mu}$ and $\left(X^{\mu}-X^{\prime \mu}\right)$. The radial momentum $p$ and angle $\theta$, together with the other $(d-2)$ angles form a spherical coordinate system in the space of momenta $p^{\mu}$. Thus, one has for the integration measure $\int d^{d} p=\Omega_{d-2} \int_{0}^{\infty} d p p^{d-1} \int_{0}^{\pi} d \theta \sin ^{d-2} \theta$, where $\Omega_{d-2}=\frac{2 \pi^{(d-1) / 2}}{\Gamma((d-1) / 2)}$ is the area of a unit radius sphere in $d-1$ dimensions. Performing the integration in Eq. (23) in this coordinate system we find

$$
K(s, w, r)=\frac{\Omega_{d-2} \sqrt{\pi}}{(2 \pi)^{d}} \frac{\Gamma\left(\frac{d-1}{2}\right)}{\left(r \sin \frac{w}{2}\right)^{(d-2) / 2}} \int_{0}^{\infty} d p p^{\frac{d}{2}} J_{\frac{d-2}{2}}\left(2 r p \sin \frac{w}{2}\right) e^{-s p^{2}} .
$$

For the trace one finds

$$
\operatorname{Tr} K(s, w)=\frac{s}{(4 \pi s)^{\frac{d}{2}}} \frac{\pi \alpha}{\sin ^{2} \frac{w}{2}} A(\Sigma),
$$

where $A(\Sigma)=\int d^{d-2} z$ is the area of the surface $\Sigma$. One uses the integral $\int_{0}^{\infty} d x x^{1-\nu} J_{\nu}(x)=\frac{2^{1-\nu}}{\Gamma(\nu)}$ for the derivation of Eq. (25). The integral over the contour $\Gamma$ in the Sommerfeld formula (22) is calculated via residues $([69,101])$

$$
C_{2}(\alpha) \equiv \frac{i}{8 \pi \alpha} \int_{\Gamma} \cot \frac{w}{2 \alpha} \frac{d w}{\sin ^{2} \frac{w}{2}}=\frac{1}{6 \alpha^{2}}\left(1-\alpha^{2}\right) .
$$

Collecting everything together one finds that in flat Minkowski spacetime

$$
\operatorname{Tr} K_{\alpha}(s)=\frac{1}{(4 \pi s)^{d / 2}}\left(\alpha V+2 \pi \alpha C_{2}(\alpha) s A(\Sigma)\right)
$$

where $V=\int d \tau d^{d-1} x$ is the volume of spacetime and $A(\Sigma)=\int d^{d-2} x$ is the area of the surface $\Sigma$. Substituting Eq. (27) into Eq. (20) we obtain that the effective action contains two terms. The one proportional to the volume $V$ reproduces the vacuum energy in the effective action. The second term proportional to the area $A(\Sigma)$ is responsible for the entropy. Applying formula (16) we obtain the entanglement entropy

$$
S=\frac{A(\Sigma)}{6(d-2)(4 \pi)^{(d-2) / 2} \epsilon^{d-2}}
$$


of an infinite plane $\Sigma$ in $d$ spacetime dimensions. Since any surface, locally, looks like a plane, and a curved spacetime, locally, is approximated by Minkowski space, this result gives the leading contribution to the entanglement entropy of any surface $\Sigma$ in flat or curved spacetime.

\subsection{Entropy of massive fields}

The heat kernel of a massive field described by the wave operator $\mathcal{D}=-\nabla^{2}+m^{2}$ is expressed in terms of the heat kernel of a massless field,

$$
K_{(m \neq 0)}\left(x, x^{\prime}, s\right)=K_{(m=0)}\left(x, x^{\prime}, s\right) \cdot e^{-m^{2} s} .
$$

Thus, one finds

$$
\operatorname{Tr} K_{\alpha}^{(m \neq 0)}(s)=\operatorname{Tr} K_{\alpha}^{(m=0)}(s) \cdot e^{-m^{2} s},
$$

where the trace of the heat kernel for vanishing mass is given by Eq. (27). Therefore, the entanglement entropy of a massive field is

$$
S_{m \neq 0}=\frac{A(\Sigma)}{12(4 \pi)^{(d-2) / 2}} \int_{\epsilon^{2}}^{\infty} \frac{d s}{s^{d / 2}} e^{-m^{2} s}
$$

In particular, if $d=4$, one finds that

$$
S_{m \neq 0}=\frac{A(\Sigma)}{12(4 \pi)}\left(\frac{1}{\epsilon^{2}}+2 m^{2} \ln \epsilon+m^{2} \ln m^{2}+m^{2}(\gamma-1)+O(\epsilon m)\right) .
$$

The logarithmic term in the entropy that is due to the mass of the field appears in any even dimension $d$. The presence of a UV finite term proportional to the $(d-2)$-th power of mass is the other general feature of (30), (31).

\subsection{An expression in terms of the determinant of the Laplacian on the surface}

Even though the entanglement entropy is determined by the geometry of the surface $\Sigma$, in general, this can be not only its intrinsic geometry but also how the surface is embedded in the larger spacetime. The embedding is determined by the extrinsic curvature. The curvature of the larger spacetime enters through the Gauss-Cadazzi relations. But in some particularly simple cases the entropy can be given a purely intrinsic interpretation. To see this for the case when $\Sigma$ is a plane we note that the entropy (28) or (30) originates from the surface term in the trace of the heat kernel (27) (or (31)). To leading order in $(1-\alpha)$, the surface term in the case of a massive scalar field is

$$
(1-\alpha) \cdot \frac{1}{6} \cdot \operatorname{Tr} K_{\Sigma}(s)
$$

where

$$
\operatorname{Tr} K_{\Sigma}(s)=\frac{A(\Sigma)}{(4 \pi s)^{\frac{d-2}{2}}} \cdot e^{-m^{2} s}
$$

can be interpreted as the trace of the heat kernel of operator $-\Delta(\Sigma)+m^{2}$, where $\Delta(\Sigma)$ is the intrinsic Laplace operator defined on the $(d-2)$-plane $\Sigma$. The determinant of the operator $-\Delta(\Sigma)+$ $m^{2}$ is determined by

$$
\ln \operatorname{det}\left(-\Delta(\Sigma)+m^{2}\right)=-\int_{\epsilon^{2}}^{\infty} \frac{d s}{s} \operatorname{Tr} K_{\Sigma}(s) .
$$


Thus, we obtain an interesting expression for the entanglement entropy

$$
S=-\frac{1}{12} \ln \operatorname{det}\left(-\Delta(\Sigma)+m^{2}\right)
$$

in terms of geometric objects defined intrinsically on the surface $\Sigma$. A similar expression in the case of an ultra-extreme black hole was obtained in [172] and for a generic black hole with horizon approximated by a plane was obtained in [89].

\subsection{Entropy in theories with a modified propagator}

In certain physically-interesting situations the propagator of a quantum field is different from the standard $1 /\left(p^{2}+m^{2}\right)$ and is described by some function as $1 / F\left(p^{2}\right)$. The quantum field in question then satisfies a modified Lorentz invariant field equation

$$
\mathcal{D} \psi=F\left(\nabla^{2}\right) \psi=0 .
$$

Theories of this type naturally arise in models with extra dimensions. The deviations from the standard form of propagator may be both in the UV regime (large values of $p$ ) or in the IR regime (small values of $p$ ). If the function $F\left(p^{2}\right)$ for large values of $p$ grows faster than $p^{2}$ this theory is characterized by improved UV behavior.

The calculation of the entanglement entropy performed in Section 2.9 can be generalized to include theories with operator (33). This example is instructive since, in particular, it illuminates the exact relation between the structure of 2-point function (the Green's function in the case of free fields) and the entanglement entropy [183].

In $d$ spacetime dimensions one has

$$
K(s, X, Y)=\frac{1}{(2 \pi)^{d}} \int d^{d} p e^{i p_{\mu}\left(X^{\mu}-Y^{\mu}\right)} e^{-s F\left(p^{2}\right)} .
$$

Note that we consider Euclidean theory so that $p^{2} \geq 0$. The Green's function

$$
G(X, Y)=<\psi(X), \psi(Y)>
$$

is a solution to the field equation with a delta-like source

$$
\mathcal{D} G(X, Y)=\delta(X, Y)
$$

and can be expressed in terms of the heat kernel as follows

$$
G(X, Y)=\int_{0}^{\infty} d s K(s, X, Y)
$$

Obviously, the Green's function can be represented in terms of the Fourier transform in a manner similar to Eq. (34),

$$
G(X, Y)=\frac{1}{(2 \pi)^{d}} \int d^{d} p e^{i p_{\mu}\left(X^{\mu}-Y^{\mu}\right)} G\left(p^{2}\right), G\left(p^{2}\right)=1 / F\left(p^{2}\right) .
$$

The calculation of the trace of the heat kernel for operator (33) on a space with a conical singularity goes along the same lines as in Section 2.9. This was performed in [184] and the result is

$$
\operatorname{Tr} K_{\alpha}(s)=\frac{1}{(4 \pi)^{d / 2}}\left(\alpha V P_{d}(s)+2 \pi \alpha C_{2}(\alpha) A(\Sigma) P_{d-2}(s)\right),
$$


where the functions $P_{n}(s)$ are defined as

$$
P_{n}(s)=\frac{2}{\Gamma\left(\frac{n}{2}\right)} \int_{0}^{\infty} d p p^{n-1} e^{-s F\left(p^{2}\right)} .
$$

The entanglement entropy takes the form (we remind the reader that for simplicity we take the surface $\Sigma$ to be a $(d-2)$-dimensional plane) [184]

$$
S=\frac{A(\Sigma)}{12 \cdot(4 \pi)^{(d-2) / 2}} \int_{\epsilon^{2}}^{\infty} \frac{d s}{s} P_{d-2}(s) \text {. }
$$

It is important to note that $[184,183]$

(i) the area law in the entanglement entropy is universal and is valid for any function $F\left(p^{2}\right)$;

(ii) the entanglement entropy is UV divergent independently of the function $F\left(p^{2}\right)$, with the degree of divergence depending on the particular function $F\left(p^{2}\right)$;

(iii) in the coincidence limit, $X=Y$, the Green's function (38)

$$
G(X, X)=\frac{2}{\Gamma\left(\frac{d}{2}\right)} \frac{1}{(4 \pi)^{\frac{d}{2}}} \int_{0}^{\infty} d p p^{d-1} G\left(p^{2}\right)
$$

may take a finite value if $G\left(p^{2}\right)=1 / F\left(p^{2}\right)$ is decaying faster than $1 / p^{d}$. However, even for this function $F\left(p^{2}\right)$, the entanglement entropy is UV divergent.

As an example, consider a function, which grows for large values of $p$ as $F\left(p^{2}\right) \sim p^{2 k}$. The 2 -point correlation function in this theory behaves as

$$
<\phi(X), \phi(Y)>\sim \frac{1}{|X-Y|^{d-2 k}}
$$

and for $k>d / 2$ it is regular in the coincidence limit. On the other hand, the entanglement entropy scales as

$$
S \sim \frac{A(\Sigma)}{\epsilon^{\frac{d-2}{k}}}
$$

and remains divergent for any positive value of $k$. Comparison of Eqs. (43) and (44) shows that only for $k=1$ (the standard form of the wave operator and the propagator) the short-distance behavior of the 2-point function is similar to the UV divergence of the entanglement entropy.

\subsection{Entanglement entropy in non-Lorentz invariant theories}

Non-Lorentz invariant theories are characterized by a modified dispersion relation, $\omega^{2}+F\left(\mathbf{p}^{2}\right)=0$, between the energy $\omega$ and the 3-momentum p. These theories can be described by a wave operator of the following type

$$
\mathcal{D}=-\partial_{t}^{2}+F\left(-\Delta_{x}\right)
$$

where $\Delta_{x}=\sum_{i}^{d-1} \partial_{i}^{2}$ is the spatial Laplace operator. Clearly, the symmetry with respect to the Lorentz boosts is broken in operator (45) if $F(q) \neq q$.

As in the Lorentz invariant case to compute the entanglement entropy associated with a surface $\Sigma$ we choose $(d-1)$ spatial coordinates $\left\{x^{i}, i=1, . ., d-1\right\}=\left\{x, z^{a}, a=1, . ., d-2\right\}$, where $x$ is the coordinate orthogonal to the surface $\Sigma$ and $z^{a}$ are the coordinates on the surface $\Sigma$. Then, after 
going to Euclidean time $\tau=i t$, we switch to the polar coordinates, $\tau=r \sin (\phi), x=r \cos (\phi)$. In the Lorentz invariant case the conical space, which is needed for calculation of the entanglement entropy, is obtained by making the angular coordinate $\phi$ periodic with period $2 \pi \alpha$ by applying the Sommerfeld formula (22) to the heat kernel. If Lorentz invariance is broken, as it is for the operator (45), there are certain difficulties in applying the method of the conical singularity when one computes the entanglement entropy. The difficulties come from the fact that the wave operator $\mathcal{D}$, if written in terms of the polar coordinates $r$ and $\phi$, becomes an explicit function of the angular coordinate $\phi$. As a result of this, the operator $\mathcal{D}$ is not invariant under shifts of $\phi$ to arbitrary $\phi+w$. Only shifts with $w=2 \pi n$, where $n$ is an integer are allowed. Thus, in this case one cannot apply the Sommerfeld formula since it explicitly uses the symmetry of the differential operator under shifts of angle $\phi$. On the other hand, a conical space with angle deficit $2 \pi(1-n)$ is exactly what we need to compute $\operatorname{Tr} \rho^{n}$ for the reduced density matrix. In [184], by using some scaling arguments it was shown that the trace of the heat kernel $K(s)=e^{-s \mathcal{D}}$ on a conical space with $2 \pi n$ periodicity, is

$$
\operatorname{Tr} K_{n}(s)=n \operatorname{Tr} K_{n=1}(s)+\frac{1}{(4 \pi)^{d / 2}} 2 \pi n C_{2}(n) A(\Sigma) P_{d-2}(s)
$$

where $n \operatorname{Tr} K_{n=1}(s)$ is the bulk contribution. By the arguments presented in Section 2.7 there is a unique analytic extension of this formula to non-integer $n$. A simple comparison with the surface term in the heat kernel of the Lorentz invariant operator, which was obtained in Section 2.12, shows that the surface terms of the two kernels are identical. Thus, we conclude that the entanglement entropy is given by the same formula

$$
S=\frac{A(\Sigma)}{12 \cdot(4 \pi)^{(d-2) / 2}} \int_{\epsilon^{2}}^{\infty} \frac{d s}{s} P_{d-2}(s),
$$

where $P_{n}(s)$ is defined in Eq. (40), as in the Lorentz invariant case (41). A similar property of the entanglement entropy was observed for a non-relativistic theory described by the Schrödinger operator [205] (see also [59] for a holographic derivation). For polynomial operators, $F(q) \sim q^{k}$, some scaling arguments can be used [205] to get the form of the entropy that follows from Eq. (47).

In the rest of the review we shall mostly focus on the study of Lorentz invariant theories, with field operator quadratic in derivatives, of the Laplace type, $\mathcal{D}=-\left(\nabla^{2}+X\right)$.

\subsection{Arbitrary surface in curved spacetime: general structure of UV divergences}

The definition of the entanglement entropy and the procedure for its calculation generalize to curved spacetime. The surface $\Sigma$ can then be any smooth closed co-dimension two surface ${ }^{2}$, which divides the space into two sub-regions. In Section 3 we will consider in detail the case where this surface is a black-hole horizon. Before proceeding to the black-hole case we would like to specify the general structure of UV divergent terms in the entanglement entropy. In $d$-dimensional curved spacetime, entanglement entropy is presented in the form of a Laurent series with respect to the UV cutoff $\epsilon$ (for $d=4$ see [204])

$$
S=\frac{s_{d-2}}{\epsilon^{d-2}}+\frac{s_{d-4}}{\epsilon^{d-4}}+. .+\frac{s_{d-2-2 n}}{\epsilon^{d-2-2 n}}+. .+s_{0} \ln \epsilon+s(g),
$$

where $s_{d-2}$ is proportional to the area of the surface $\Sigma$. All other terms in the expansion (48) can be presented as integrals over $\Sigma$ of local quantities constructed in terms of the Riemann curvature of

\footnotetext{
${ }^{2}$ If the boundary of $\Sigma$ is not empty there could be extra terms in the entropy proportional to the "area" of the
} boundary $\partial \Sigma$ as was shown in [108]. We do not consider this case here.

Living Reviews in Relativity

http: //www . livingreviews . org/lrr-2011-8 
the spacetime and the extrinsic curvature of the surface $\Sigma$. Of course, the intrinsic curvature of the surface $\Sigma$ can be expressed in terms of $\mathcal{R}$ and $k$ using the Gauss-Codazzi equations. Since nothing should depend on the direction of vectors normal to $\Sigma$, the integrands in expansion (48) should be even powers of extrinsic curvature. The general form of the $s_{d-2-2 n}$ term can be symbolically presented in the form

$$
s_{d-2-2 n}=\sum_{l+p=n} \int_{\Sigma} \mathcal{R}^{l} k^{2 p},
$$

where $\mathcal{R}$ stands for components of the Riemann tensor and their projections onto the sub-space orthogonal to $\Sigma$ and $k$ labels the components of the extrinsic curvature. Thus, since the integrands are even in derivatives, only terms $\epsilon^{d-2 n-2}, n=0,1,2, \ldots$ appear in Eq. (48). If $d$ is even, then there also may appear a logarithmic term $s_{0}$. The term $s(g)$ in Eq. (48) is a UV finite term, which may also depend on the geometry of the surface $\Sigma$, as well as on the geometry of the spacetime itself. 


\section{Entanglement Entropy of Non-Degenerate Killing Hori- zons}

\subsection{The geometric setting of black-hole spacetimes}

The notion of entanglement entropy is naturally applicable to a black hole. In fact, probably the only way to separate a system into two sub-systems is to place one of them inside a black-hole horizon. The important feature that, in fact, defines the black hole is the existence of a horizon. Many useful definitions of a horizon are known. In the present paper we shall consider only the case of the eternal black holes for which different definitions of the horizon coincide. The corresponding spacetime then admits a maximal analytic extension, which we shall use in our construction. The simplest example is the Schwarzschild black hole, the maximal extension of which is demonstrated on the well-known Penrose diagram. The horizon of the Schwarzschild black hole is an example of a Killing horizon. The spacetime in this case possesses a global Killing vector, $\xi_{t}=\partial_{t}$, which generates the time translations. The Killing horizon is defined as a null hypersurface on which the Killing vector $\xi_{t}$ is null, $\xi_{t}^{2}=0$. The null surface in the maximal extension of an eternal black hole consists of two parts: the future horizon and the past horizon. The two intersect on a compact surface of co-dimension two, $\Sigma$, called the bifurcation surface. In the maximally extended spacetime a hypersurface $\mathcal{H}_{t}$ of constant time $t$ is a Cauchy surface. The bifurcation surface $\Sigma$ naturally splits the Cauchy surface into two parts, $\mathcal{H}_{-}$and $\mathcal{H}_{+}$, respectively inside and outside the black hole. For asymptotically-flat spacetime, such as the Schwarzschild metric, the hypersurface $\mathcal{H}_{t}$ has the topology of a wormhole. (In the case of the Schwarzschild metric it is called the Einstein-Rosen bridge.) The surface $\Sigma$ is the surface of minimal area in $\mathcal{H}_{t}$. In fact the bifurcation surface $\Sigma$ is a minimal surface not only in the $(d-1)$-dimensional Euclidean space $\mathcal{H}_{t}$, but also in the $d$-dimensional spacetime. As a consequence, as we show below, the components of the extrinsic curvature defined for two vectors normal to $\Sigma$, vanish on $\Sigma$.

The spacetime in question admits a Euclidean version by analytic continuation $t \rightarrow i \tau$. It is a feature of regular metrics with a Killing horizon that the direction of Euclidean time $\tau$ is compact with period $2 \pi \beta_{H}$, which is determined by the condition of regularity, i.e., the absence of a conical singularity. In a vicinity of the bifurcation surface $\Sigma$, the spacetime then is a product of a compact surface $\Sigma$ and a two-dimensional disk, the time coordinate $\tau$ playing the role of the angular coordinate on the disk. The latter can be made more precise by introducing a new angular variable $\phi=\beta_{H}^{-1} \tau$, which varies from 0 to $2 \pi$. In this paper we consider the spacetime with Euclidean metric of the general type

$$
d s^{2}=\beta_{H}^{2} g(\rho) d \phi^{2}+d \rho^{2}+\gamma_{i j}(\rho, \theta) d \theta^{i} d \theta^{j} .
$$

The radial coordinate $\rho$ is such that the surface $\Sigma$ is defined by the condition $\rho=0$. Near this point the functions $g(\rho)$ and $\gamma_{i j}(\rho, \theta)$ can be expanded as

$$
g(\rho)=\frac{\rho^{2}}{\beta_{H}^{2}}+O\left(\rho^{4}\right), \gamma_{i j}(\rho, \theta)=\gamma_{i j}^{(0)}(\theta)+O\left(\rho^{2}\right),
$$

where $\gamma_{i j}^{(0)}(\theta)$ is the metric on the bifurcation surface $\Sigma$ equipped with coordinates $\left\{\theta^{i}, i=1, . ., d-\right.$ $2\}$. This metric describes what is called a non-degenerate horizon. The Hawking temperature of the horizon is finite in this case and equal to $T_{H}=1 /\left(2 \pi \beta_{H}\right)$.

It is important to note that the metric (50) does not have to satisfy any field equations. The entanglement entropy can be defined for any metric, which possesses a Killing-type horizon. In this sense the entanglement entropy is an off-shell quantity. It is useful to keep this in mind when one compares the entanglement entropy with some other approaches in which an entropy is assigned to a black hole horizon. Even though the metric (50) with (51) does not have to satisfy

Living Reviews in Relativity

http: //www. livingreviews.org/lrr-2011-8 
the Einstein equations we shall still call the complete space described by the Euclidean metric (50) the Euclidean black hole instanton and will denote it by $E$.

\subsection{Extrinsic curvature of horizon, horizon as a minimal surface}

The horizon surface $\Sigma$ defined by the condition $\rho=0$ in the metric (50) is a co-dimension 2 surface. It has two normal vectors: a spacelike vector $n^{1}$ with the only non-vanishing component $n_{\rho}^{1}=1$ and a timelike vector $n^{2}$ with the non-vanishing component $n_{\phi}^{2}=1 / \rho$. With respect to each normal vector one defines an extrinsic curvature, $k_{i j}^{a}=-\gamma_{i}{ }^{l} \gamma_{j}{ }^{p} \nabla_{l} n_{p}^{a}, a=1,2$. The extrinsic curvature $k_{i j}^{2}$ identically vanishes. It is a consequence of the fact that $n^{2}$ is a Killing vector, which generates time translations. Indeed, the extrinsic curvature can be also written as a Lie derivative, $k_{\mu \nu}=-\frac{1}{2} \mathcal{L}_{n} g_{\mu \nu}$, so that it vanishes if $n$ is a Killing vector. The extrinsic curvature associated to the vector $n^{1}$,

$$
k_{i j}^{1}=-\frac{1}{2} \gamma_{i}^{l} \gamma_{j}^{p} \partial_{\rho} \gamma_{k n},
$$

is vanishing when restricted to the surface defined by the condition $\rho=0$. It is due to the fact that the term linear in $\rho$ is absent in the $\rho$-expansion for $\gamma_{i j}(\rho, \theta)$ in the metric $(50)$. This is required by the regularity of the metric (50): in the presence of such a term the Ricci scalar would be singular at the horizon, $R \sim 1 / \rho$.

The vanishing of the extrinsic curvature of the horizon indicates that the horizon is necessarily a minimal surface. It has the minimal area considered as a surface in $d$-dimensional spacetime. On the other hand, in the Lorentzian signature, the horizon $\Sigma$ has the minimal area if considered on the hypersurface of constant time $t, \mathcal{H}_{t}$; thus, the latter has the topology of a wormhole.

\subsection{The wave function of a black hole}

Although the entanglement entropy can be defined for any co-dimension two surface, when the surface is a horizon particular care is required. In order to apply the general prescription outlined in Section 2.1, we first of all need to specify the corresponding wave function. Here we will follow the prescription proposed by Barvinsky, Frolov and Zelnikov [15]. This prescription is a natural generalization of the one in flat spacetime discussed in Section 3.8. On the other hand, it is similar to the "no-boundary" wave function of the universe introduced in [127]. We define the wave function of a black hole by the Euclidean path integral over field configurations on the half-period Euclidean instanton defined by the metric (50) with angular coordinate $\phi$ changing in the interval from 0 to $\pi$. This half-period instanton has Cauchy surface $\mathcal{H}$ (on which we can choose coordinates $x=(\rho, \theta))$ as a boundary where we specify the boundary conditions in the path integral,

$$
\begin{aligned}
\Psi\left[\psi_{-}(x), \psi_{+}(x)\right]= & \int \\
& \mathcal{D} \psi e^{-W[\psi]}, \\
& \left.\psi(X)\right|_{\phi=0}=\psi_{+}(x) \\
&
\end{aligned}
$$

where $W[\psi]=\frac{1}{2} \int \psi \hat{\mathcal{D}} \psi$ is the action of the quantum field $\psi$. The functions $\psi_{-}(x)$ and $\psi_{+}(x)$ are the boundary values defined on the part of the hypersurface $\mathcal{H}$, which is respectively inside $\left(\mathcal{H}_{-}\right)$ and outside $\left(\mathcal{H}_{+}\right)$the horizon $\Sigma$. As was shown in [15], the wave function (53) corresponds to the Hartle-Hawking vacuum state [126].

\subsection{Reduced density matrix and entropy}

The density matrix $\rho\left(\psi_{+}^{1}, \psi_{+}^{2}\right)$ defined by tracing over $\psi_{-}$-modes is given by the Euclidean path integral over field configurations on the complete instanton $(0<\phi<2 \pi)$ with a cut along the axis 
$\phi=0$ where the field $\psi(X)$ in the path integral takes the values $\psi_{+}^{1}(x)$ and $\psi_{+}^{2}(x)$ below and above the cut respectively. The trace $\operatorname{Tr} \rho$ is obtained by equating the fields across the cut and doing the unrestricted Euclidean path integral on the complete Euclidean instanton E. Analogously, $\operatorname{Tr} \rho^{n}$ is given by the path integral over field configurations defined on the n-fold cover $E_{n}$ of the complete instanton. This space is described by the metric (50) where angular coordinate $\phi$ is periodic with period $2 \pi n$. It has a conical singularity on the surface $\Sigma$ so that in a small vicinity of $\Sigma$ the total space $E_{n}$ is a direct product of $\Sigma$ and a two-dimensional cone $\mathcal{C}_{n}$ with angle deficit $\delta=2 \pi(1-n)$. Due to the abelian isometry generated by the Killing vector $\partial_{\phi}$ this construction can be analytically continued to arbitrary (non-integer) $n \rightarrow \alpha$. So that one can define a partition function

$$
Z(\alpha)=\operatorname{Tr} \rho^{\alpha}
$$

by the path integral over field configurations over $E_{\alpha}$, the $\alpha$-fold cover of the instanton $E$. For a bosonic field described by the field operator $\hat{\mathcal{D}}$ one has that $Z(\alpha)=\operatorname{det}^{-1 / 2} \hat{\mathcal{D}}$. Defining the effective action as $W(\alpha)=-\ln Z(\alpha)$, the entanglement entropy is still given by formula (16), i.e., by differentiating the effective action with respect to the angle deficit. Clearly, only the term linear in $(1-\alpha)$ contributes to the entropy. Thus, the problem reduces to the calculation of this term in the effective action.

\subsection{The role of the rotational symmetry}

We emphasize that the presence of the rotational symmetry with respect to the Killing vector $\partial_{\phi}$, which generates rotations in the 2-plane orthogonal to the entangling surface $\Sigma$, plays an important role in our construction. Indeed, without such a symmetry it would be impossible to interpret $\operatorname{Tr} \rho^{\alpha}$ for an arbitrary $\alpha$ as a partition function in some gravitational background. In general, two points are important for this interpretation:

i) that the spacetime possesses, at least locally near the entangling surface, a rotational symmetry so that, after the identification $\phi \rightarrow \phi+2 \pi \alpha$, we get a well-defined spacetime $E_{\alpha}$, with no more than just a conical singularity; this holds automatically if the surface in question is a Killing horizon;

ii) and that the field operator is invariant under the "rotations", $\phi \rightarrow \phi+w$; this is automatic if the field operator is a covariant operator.

In particular, point ii) allows us to use the Sommerfeld formula (more precisely its generalization to a curved spacetime) in order to define the Green's function or the heat kernel on the space $E_{\alpha}$. As is shown in [184] (see also discussion in Section 2.13) in the case of the non-Lorentz invariant field operators in flat Minkowski spacetime, the lack of the symmetry ii) makes the whole "conical space" approach rather obscure. On the other hand, in the absence of rotational symmetry i) there may appear terms in the entropy that are "missing" in the naively applied conical space approach: the extrinsic curvature contributions [204] or even some curvature terms [134].

In what follows we consider the entanglement entropy of the Killing horizons and deal with the covariant operators so that we do not have to worry about i) or ii).

\subsection{Thermality of the reduced density matrix of a Killing horizon}

The quantum state defined by Eq. (53) is the Hartle-Hawking vacuum [126]. The Green's function in this state is defined by analytic continuation from the Euclidean Green's function. The periodicity $t \rightarrow t+i \beta_{H}$ is thus inherent in this state. This periodicity indicates that the correlation functions computed in this state are in fact thermal correlation functions when continued to the Lorentzian section. This fact generalizes to an arbitrary interacting quantum field as shown in [121]. On the other hand, being globally defined, the Hartle-Hawking state is a pure state, which

Living Reviews in Relativity

http: //www . livingreviews . org/lrr-2011-8 
involves correlations between modes localized on different sides of the horizon. However, this state is described by a thermal density matrix if reduced to modes defined on one side of the horizon as was shown by Israel [138]. That the reduced density matrix obtained by tracing over modes inside the horizon is thermal can be formally seen by using angular quantization. Introducing the Euclidean Hamiltonian $H_{E}$, which is the generator of rotations with respect to the angular coordinate $\phi$ defined above, one finds that $\rho\left(\psi_{+}^{1}, \psi_{+}^{2}\right)=<\psi_{+}^{1}\left|e^{-2 \pi H_{E}}\right| \psi_{+}^{2}>$, i.e., the density matrix is thermal with respect to the Hamiltonian $H_{E}$ with inverse temperature $2 \pi$. This formal proof in Minkowski space was outlined in [152]. The appropriate Euclidean Hamiltonian is then the Rindler Hamiltonian, which generates Lorentz boosts in a direction orthogonal to the surface $\Sigma$. In [142] the proof was generalized to the case of generic static spacetimes with bifurcate Killing horizons admitting a regular Euclidean section.

\subsection{Useful mathematical tools}

\subsubsection{Curvature of space with a conical singularity}

Consider a space $E_{\alpha}$, which is an $\alpha$-fold covering of a smooth manifold $E$ along the Killing vector $\partial_{\varphi}$, generating an abelian isometry. Let surface $\Sigma$ be a stationary point of this isometry so that near $\Sigma$ the space $E_{\alpha}$ looks like a direct product, $\Sigma \times \mathcal{C}_{\alpha}$, of the surface $\Sigma$ and a two-dimensional cone $\mathcal{C}_{\alpha}$ with angle deficit $\delta=2 \pi(1-\alpha)$. Outside the singular surface $\Sigma$ the space $E_{\alpha}$ has the same geometry as a smooth manifold $E$. In particular, their curvature tensors coincide. However, the conical singularity at the surface $\Sigma$ produces a singular (delta-function like) contribution to the curvatures. This was first demonstrated by Sokolov and Starobinsky [195] in the two-dimensional case by using topological arguments. These arguments were generalized to higher dimensions in [7]. One way to extract the singular contribution is to use some regularization procedure, replacing the singular space $E_{\alpha}$ by a sequence of regular manifolds $\tilde{E}_{\alpha}$. This procedure was developed by Fursaev and Solodukhin in [111]. In the limit $\tilde{E}_{\alpha} \rightarrow E_{\alpha}$ one obtains the following results [111]:

$$
\begin{aligned}
R_{\alpha \beta}^{\mu \nu} & =\bar{R}^{\mu \nu}{ }_{\alpha \beta}+2 \pi(1-\alpha)\left(\left(n^{\mu} n_{\alpha}\right)\left(n^{\nu} n_{\beta}\right)-\left(n^{\mu} n_{\beta}\right)\left(n^{\nu} n_{\alpha}\right)\right) \delta_{\Sigma}, \\
R_{\nu}^{\mu} & =\bar{R}^{\mu}{ }_{\nu}+2 \pi(1-\alpha)\left(n^{\mu} n_{\nu}\right) \delta_{\Sigma}, \\
R & =\bar{R}+4 \pi(1-\alpha) \delta_{\Sigma},
\end{aligned}
$$

where $\delta_{\Sigma}$ is the delta-function, $\int_{\mathcal{M}} f \delta_{\Sigma}=\int_{\Sigma} f ; n^{k}=n_{k}^{\mu} \partial_{\mu}, k=1,2$ are two orthonormal vectors orthogonal to the surface $\Sigma,\left(n_{\mu} n_{\nu}\right)=\sum_{k=1}^{2} n_{\mu}^{k} n_{\nu}^{k}$ and the quantities $\bar{R}_{\alpha \beta}^{\mu \nu}, \bar{R}_{\nu}^{\mu}$ and $\bar{R}$ are computed in the regular points $E_{\alpha} / \Sigma$ by the standard method.

These formulas can be used to define the integral expressions ${ }^{3}$ [11]

$$
\begin{aligned}
\int_{E_{\alpha}} R & =\alpha \int_{E} \bar{R}+4 \pi(1-\alpha) \int_{\Sigma} 1, \\
\int_{E_{\alpha}} R^{2} & =\alpha \int_{E} \bar{R}^{2}+8 \pi(1-\alpha) \int_{\Sigma} \bar{R}+O\left((1-\alpha)^{2}\right), \\
\int_{E_{\alpha}} R^{\mu \nu} R_{\mu \nu} & =\alpha \int_{E} \bar{R}^{\mu \nu} \bar{R}_{\mu \nu}+4 \pi(1-\alpha) \int_{\Sigma} \bar{R}_{i i}+O\left((1-\alpha)^{2}\right), \\
\int_{E_{\alpha}} R^{\mu \nu \lambda \rho} R_{\mu \nu \lambda \rho} & =\alpha \int_{E} \bar{R}^{\mu \nu \lambda \rho} \bar{R}_{\mu \nu \lambda \rho}+8 \pi(1-\alpha) \int_{\Sigma} \bar{R}_{i j i j}+O\left((1-\alpha)^{2}\right),
\end{aligned}
$$

where $\bar{R}_{i i}=\bar{R}_{\mu \nu} n_{i}^{\mu} n_{i}^{\nu}$ and $\bar{R}_{i j i j}=\bar{R}_{\mu \nu \lambda \rho} n_{i}^{\mu} n_{i}^{\lambda} n_{j}^{\nu} n_{j}^{\rho}$. We use a shorthand notation for the surface integral $\int_{\Sigma} \equiv \int_{\Sigma} \sqrt{\gamma} d^{d-2} \theta$.

\footnotetext{
${ }^{3}$ It should be noted that formulas (55), (56), (57), (58), and (59) are valid even if subleading terms (as in Eq. (51)) in the expansion of the metric near the singular surface $\Sigma$ are functions of $\theta$ [111]. Such more general metrics describe what might be called a "local Killing horizon".
} 
The terms proportional to $\alpha$ in Eqs. (56) - (59) are defined on the regular space $E$. The terms $O\left((1-\alpha)^{2}\right)$ in Eqs. (57)-(59) are something like a square of the $\delta$-function. They are not welldefined and depend on the way the singular limit $\tilde{E}_{\beta} \rightarrow \mathcal{E}_{\beta}$ is taken. However, those terms are not important in the calculation of the entropy since they are of higher order in $(1-\alpha)$. However, there are certain invariants, polynomial in the Riemann tensor, in which the terms $O\left((1-\alpha)^{2}\right)$ do not appear at all. Thus, these invariants are well defined on the manifolds with conical singularity. Below we consider two examples of such invariants [111].

Topological Euler number. The topological Euler number of a $2 p$-dimensional smooth manifold $\mathcal{E}$ is given by the integral ${ }^{4}$

$$
\begin{aligned}
& \chi=\int_{E} \mathcal{E}_{2 p} \sqrt{g} d^{2 p} x, \\
& \mathcal{E}_{2 p}=c_{p} \epsilon_{\mu_{1} \mu_{2} \ldots \mu_{2 p-1} \mu_{2 p}} \epsilon^{\nu_{1} \nu_{2} \ldots \nu_{2 p-1} \nu_{2 p}} R_{\nu_{1} \nu_{2}}^{\mu_{1} \mu_{2}} \ldots R_{\substack{\nu_{2 p-1} \nu_{2 p} \\
\mu_{2 p-1} \mu_{2 p}}, c_{p}=\frac{1}{2^{3 p} \pi p !} .} .
\end{aligned}
$$

Suppose that $E_{\alpha}$ has several singular surfaces (of dimension $2(p-1)$ ) $\Sigma_{i}$, each with conical deficit $2 \pi\left(1-\alpha_{i}\right)$, then the Euler characteristic of this manifold is [111]

$$
\chi\left[E_{\alpha}\right]=\int_{E_{\alpha} / \Sigma} \mathcal{E}_{2 p}+\sum_{i}\left(1-\alpha_{i}\right) \chi\left[\Sigma_{i}\right] .
$$

A special case is when $E_{\alpha}$ possesses a continuous abelian isometry. The singular surfaces $\Sigma_{i}$ are the fixed points of this isometry so that all surfaces have the same angle deficit $\alpha_{i}=\alpha$. The Euler number in this case is [111]

$$
\chi\left[E_{\alpha}\right]=\alpha \chi\left[E_{\alpha=1}\right]+(1-\alpha) \sum_{i} \chi\left[\Sigma_{i}\right] .
$$

An interesting consequence of this formula is worth mentioning. Since the introduction of a conical singularity can be considered as the limit of certain smooth deformation, under which the topological number does not change, one has $\chi\left[E_{\alpha}\right]=\chi\left[E_{\alpha=1}\right]$. Then one obtains an interesting formula reducing the number $\chi$ of a manifold $E$ to that of the fixed points set of its abelian isometry [111]

$$
\chi\left[E_{\alpha=1}\right]=\sum_{i} \chi\left[\Sigma_{i}\right]
$$

A simple check shows that Eq. (63) gives the correct result for the Euler number of the sphere $S_{\alpha}^{d}$. Indeed, the fixed points of 2-sphere $S_{\alpha}^{2}$ are its "north" and "south" poles. Each of these points has $\chi=1$ and one gets from Eq. (63): $\chi\left[S^{2}\right]=1+1=2$. On the other hand, the singular surface of $S_{\alpha}^{d}(d \geq 3)$ is $S^{d-2}$ and from Eq. (63) the known identity $\chi\left[S^{d}\right]=\chi\left[S^{d-2}\right]$ follows. Note that Eq. (63) is valid for spaces with continuous abelian isometry and it may be violated for an orbifold with conical singularities.

Lovelock gravitational action. The general Lovelock gravitational action is introduced on a d-dimensional Riemannian manifold as the following polynomial [166]

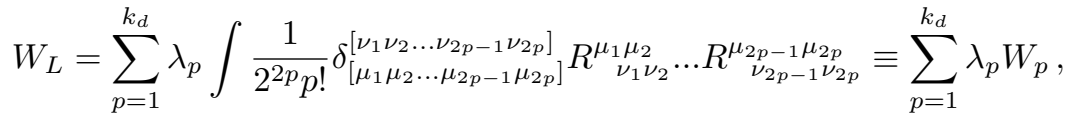

\footnotetext{
${ }^{4}$ Note that in [111] there is a typo in Eq. (3.9) defining $c_{p}$. This does not affect the conclusions of [111] since they are based on the relation $c_{p-1}=8 \pi p c_{p}$ rather than on the explicit form of $c_{p}$.
} 
where $\delta_{[\ldots]}^{[\ldots]}$ is the totally antisymmetrized product of the Kronecker symbols and $k_{d}$ is $(d-2) / 2$ (or $(d-1) / 2$ ) for even (odd) dimension $d$. If the dimension of spacetime is $2 p$, the action $W_{p}$ reduces to the Euler number (60) and is thus topological. In other dimensions the action (64) is not topological, although it has some nice properties, which make it interesting. In particular, the field equations, which follow from Eq. (64), are quadratic in derivatives even though the action itself is polynomial in curvature.

On a conical manifold $\mathcal{M}_{\alpha}$, the Lovelock action is the sum of volume and surface parts [111]

$$
W_{L}\left[\mathcal{M}_{\alpha}\right]=W_{L}\left[\mathcal{M}_{\alpha} / \Sigma\right]+2 \pi(1-\alpha) \sum_{p=0}^{k_{d}-1} \lambda_{p+1} W_{p}[\Sigma]
$$

where the first term is the action computed at the regular points. As in the case of the topological Euler number, all terms quadratic in $(1-\alpha)$ mutually cancel in Eq. (65). The surface term in Eq. (65) takes the form of the Lovelock action on the singular surface $\Sigma$. It should be stressed that integrals $W_{p}[\Sigma]$ are defined completely in terms of the intrinsic Riemann curvature $R_{k n}^{i j}$ of $\Sigma$

$$
W_{p}[\Sigma]=\frac{1}{2^{2 p} p !} \int_{\Sigma} \delta_{\left[j_{1} \ldots j_{2 p}\right]}^{\left[i_{1} \ldots i_{2 p}\right]} R_{j_{1} j_{2}}^{i_{1} i_{2}} \underset{j_{2 p-1} R_{2 p}}{i_{2 p-1} i_{2 p}}
$$

and $W_{0} \equiv \int_{\Sigma}$. Eq. (65) allows us to compute the entropy in the Lovelock gravity by applying the replica formula. In [145] this entropy was derived in the Hamiltonian approach, whereas arguments based on the dimensional continuation of the Euler characteristics have been used for its derivation in $[7]$.

\subsubsection{The heat kernel expansion on a space with a conical singularity}

The useful tool to compute the effective action on a space with a conical singularity is the heat kernel method already discussed in Section 2.8. In Section 2.9 we have shown how, in flat space, using the Sommerfeld formula (22), to compute the contribution to the heat kernel due to the singular surface $\Sigma$. This calculation can be generalized to an arbitrary curved space $E_{\alpha}$ that possesses, at least locally, an abelian isometry with a fixed point. To be more specific we consider a scalar field operator $\mathcal{D}=-\left(\nabla^{2}+X\right)$, where $X$ is some scalar function. Then, the trace of the heat kernel $K=e^{-s \mathcal{D}}$ has the following small $s$ expansion

$$
\operatorname{Tr} K_{E_{\alpha}}(s)=\frac{1}{(4 \pi s)^{\frac{d}{2}}} \sum_{n=0} a_{n} s^{n},
$$

where the coefficients in the expansion decompose into bulk (regular) and surface (singular) parts

$$
a_{n}=a_{n}^{\mathrm{reg}}+a_{n}^{\Sigma} .
$$

The regular coefficients are the same as for a smooth space. The first few coefficients are

$$
\begin{aligned}
& a_{0}^{\mathrm{reg}}=\int_{E_{\alpha}} 1, \quad a_{1}^{\mathrm{reg}}=\int_{E_{\alpha}}\left(\frac{1}{6} \bar{R}+X\right) \\
& a_{2}^{\mathrm{reg}}=\int_{E_{\alpha}}\left(\frac{1}{180} \bar{R}_{\mu \nu \alpha \beta}^{2}-\frac{1}{180} \bar{R}_{\mu \nu}^{2}+\frac{1}{6} \nabla^{2}\left(X+\frac{1}{5} \bar{R}\right)+\frac{1}{2}\left(X+\frac{1}{6} \bar{R}\right)^{2}\right) .
\end{aligned}
$$

The coefficients due to the singular surface $\Sigma$ (the stationary point of the isometry) are

$$
\begin{aligned}
& a_{0}^{\Sigma}=0 ; \quad a_{1}^{\Sigma}=\frac{\pi}{3} \frac{(1-\alpha)(1+\alpha)}{\alpha} \int_{\Sigma} 1 \\
& a_{2}^{\Sigma}=\frac{\pi}{3} \frac{(1-\alpha)(1+\alpha)}{\alpha} \int_{\Sigma}\left(\frac{1}{6} \bar{R}+X\right)-\frac{\pi}{180} \frac{(1-\alpha)(1+\alpha)\left(1+\alpha^{2}\right)}{\alpha^{3}} \int_{\Sigma}\left(\bar{R}_{i i}-2 \bar{R}_{i j i j}\right) .
\end{aligned}
$$


The form of the regular coefficients (69) in the heat kernel expansion has been well studied in physics and mathematics literature (for a review see [219]). The surface coefficient $a_{1}^{\Sigma}$ in Eq. (70) was calculated by the mathematicians McKean and Singer [174] (see also [42]). In physics literature this term has appeared in the work of Dowker [69]. (In the context of cosmic strings one has focused more on the Green's function rather on the heat kernel [3, 100].) The coefficient $a_{2}^{\Sigma}$ was first obtained by Fursaev [101] although in some special cases it was known before in works of Donnelly [64, 65].

It should be noted that due to the fact that the $\operatorname{surface} \Sigma$ is a fixed point of the abelian isometry, all components of the extrinsic curvature of the surface $\Sigma$ vanish. This explains why the extrinsic curvature does not appear in the surface terms (70) in the heat kernel expansion.

\subsection{General formula for entropy in the replica method, relation to the Wald entropy}

As a consequence of the expressions (55) for the curvature of space with a conical singularity that were presented in Section 3.7.1 one obtains a general expression for the entropy. Consider a Euclidean general covariant action

$$
W\left[g_{\mu \nu}, \varphi_{A}\right]=-\int d^{d} x \sqrt{g} \mathcal{L}\left(g_{\mu \nu}, R_{\mu \nu}^{\alpha \beta}, \nabla_{\sigma} R_{\mu \nu}^{\alpha \beta}, \ldots, \varphi_{A}\right),
$$

which describes the gravitational field coupled to some matter fields $\varphi_{A}$. In the replica trick we first introduce a conical singularity at the horizon surface $\Sigma$ with a small angle deficit $\delta=2 \pi(1-\alpha)$ so that the Riemann curvature obtains a delta-like surface contribution (55) and the gravitational action (71) becomes a function of $\alpha$. Then applying the replica formula

$$
S=\left.\left(\alpha \partial_{\alpha}-1\right) W(\alpha)\right|_{\alpha=1}
$$

we get

$$
S=2 \pi \int_{\Sigma} Q_{\alpha \beta \mu \nu}\left(\left(n^{\mu} n^{\alpha}\right)\left(n^{\nu} n^{\beta}\right)-\left(n^{\mu} n^{\beta}\right)\left(n^{\nu} n^{\alpha}\right)\right)
$$

for the entropy associated to $\Sigma$, where tensor $Q_{\alpha \beta \mu \nu}$ is defined as a variation of action (71) with respect to the Riemann tensor,

$$
Q_{\alpha \beta}^{\mu \nu}=\frac{1}{\sqrt{g}} \frac{\delta W\left[g_{\mu \nu}, \varphi_{A}\right]}{\delta R_{\mu \nu}^{\alpha \beta}} .
$$

If action (71) is local and it does not contain covariant derivatives of the Riemann tensor, then the tensor $Q_{\alpha \beta}^{\mu \nu}$ is a partial derivative of the Lagrangian,

$$
Q_{\alpha \beta}^{\mu \nu}=\frac{\partial \mathcal{L}}{\partial R_{\mu \nu}^{\alpha \beta}}
$$

Now, as was observed by Myers and Sinha [181] (see also [4]), one can re-express

$$
\sum_{i, j=1}^{2}\left(n_{i}^{\mu} n_{i}^{\alpha}\right)\left(n_{j}^{\nu} n_{j}^{\beta}\right)-\left(n_{i}^{\mu} n_{i}^{\beta}\right)\left(n_{j}^{\nu} n_{j}^{\alpha}\right)=\epsilon^{\mu \nu} \epsilon^{\alpha \beta},
$$

where $\epsilon^{\alpha \beta}=n_{1}^{\alpha} n_{2}^{\beta}-n_{2}^{\alpha} n_{1}^{\beta}$ is the two-dimensional volume form in the space transverse to the horizon surface $\Sigma$. Then, for a local action (71) polynomial in the Riemann curvature, the entropy (72) takes the form

$$
S=2 \pi \int_{\Sigma} \frac{\partial \mathcal{L}}{\partial R_{\mu \nu}^{\alpha \beta}} \epsilon_{\mu \nu} \epsilon^{\alpha \beta}
$$


which is exactly the Wald entropy [221, 144]. It should be noted that Wald's Noether charge method is an on-shell method so that the metric in the expression for the Wald entropy is supposed to satisfy the field equations. On the other hand, the conical singularity method is an off-shell method valid for any metric that describes a black-hole horizon. The relation between the on-shell and the off-shell descriptions will be discussed in Section 4.1.

\subsection{UV divergences of entanglement entropy for a scalar field}

For a bosonic field described by a field operator $\mathcal{D}$ the partition function is $Z(\alpha)=\operatorname{det}^{-1 / 2} \mathcal{D}$. The corresponding effective action $W(\alpha)=-\ln Z(\alpha)$ on a space with a conical singularity, $E_{\alpha}$, is expressed in terms of the heat kernel $K_{E_{\alpha}}(s)$ in a standard way

$$
W(\alpha)=-\frac{1}{2} \int_{\epsilon^{2}}^{\infty} \frac{d s}{s} \operatorname{Tr} K_{E_{\alpha}}(s)
$$

The entanglement entropy is computed using the replica trick as

$$
S=\left.\left(\alpha \partial_{\alpha}-1\right) W(\alpha)\right|_{\alpha=1}
$$

Using the small $s$ expansion one can, in principle, compute all UV divergent terms in the entropy. However, the surface terms are known only for the first few terms in the expansion (67). This allows us to derive an explicit form for the UV divergent terms in the entropy.

In two dimensions the horizon is just a point and the entanglement entropy diverges logarithmically [33, 152, 71, 85, 196]

$$
S_{d=2}=\frac{1}{6} \ln \frac{1}{\epsilon}
$$

In three dimensions the horizon is a circle and the entropy

$$
S_{d=3}=\frac{A(\Sigma)}{12 \sqrt{\pi} \epsilon}
$$

is linearly divergent.

The leading UV divergence in $\boldsymbol{d}$ dimensions can be computed directly by using the form of the coefficient $a_{1}^{\Sigma}(70)$ in the heat kernel expansion [33]

$$
S_{d}=\frac{1}{6(d-2)(4 \pi)^{\frac{d-2}{2}}} \frac{A(\Sigma)}{\epsilon^{d-2}} .
$$

It is identical to expression (28) for the entanglement entropy in flat Minkowski spacetime. This has a simple explanation. To leading order the spacetime near the black-hole horizon is approximated by the flat Rindler metric. Thus, the leading UV divergent term in the entropy is the entanglement entropy of the Rindler horizon. The curvature corrections then show up in the subleading UV divergent terms and in the UV finite terms. 
The four-dimensional case is the most interesting since in this dimension there appears a logarithmic subleading term in the entropy. For a scalar field described by a field operator $-\left(\nabla^{2}+\right.$ $X)$ the UV divergent terms in the entanglement entropy of a generic 4-dimensional black hole read [197]

$$
S_{d=4}=\frac{A(\Sigma)}{48 \pi \epsilon^{2}}-\frac{1}{144 \pi} \int_{\Sigma}\left(R+6 X-\frac{1}{5}\left(R_{i i}-2 R_{i j i j}\right)\right) \ln \epsilon .
$$

We note that for a massive scalar field $X=-m^{2}$.

Of special interest is the case of the $4 \mathrm{~d}$ conformal scalar field. In this case $X=-\frac{1}{6} R$ and the entropy (82) takes the form

$$
S_{\mathrm{conf}}=\frac{A(\Sigma)}{48 \pi \epsilon^{2}}+\frac{1}{720 \pi} \int_{\Sigma}\left(R_{i i}-2 R_{i j i j}\right) \ln \epsilon
$$

The logarithmic term in Eq. (83) is invariant under the simultaneous conformal transformations of bulk metric $g_{\mu \nu} \rightarrow e^{2 \sigma} g_{\mu \nu}$ and the metric on the surface $\Sigma, \gamma_{i j} \rightarrow e^{2 \sigma} \gamma_{i j}$. This is a general feature of the logarithmic term in the entanglement entropy of a conformally-invariant field.

Let us consider some particular examples.

\subsubsection{The Reissner-Nordström black hole}

A black hole of particular interest is the charged black hole described by the Reissner-Nordström metric,

$$
\begin{aligned}
& d s_{\mathrm{RN}}^{2}=g(r) d \tau^{2}+g^{-1}(r) d r^{2}+r^{2}\left(d \theta^{2}+\sin ^{2} \theta d \phi^{2}\right), \\
& g(r)=1-\frac{\left(r-r_{+}\right)\left(r-r_{-}\right)}{r^{2}} .
\end{aligned}
$$

This metric has a vanishing Ricci scalar, $\bar{R}=0$. It has inner and out horizons, $r_{-}$and $r_{+}$ respectively, defined by

$$
r_{ \pm}=m \pm \sqrt{m^{2}-q^{2}}
$$

where $m$ is the mass of the black hole and $q$ is the electric charge of the black hole. The two vectors normal to the horizon are characterized by the non-vanishing components $n_{1}^{\tau}=g^{-1 / 2}(r)$, $n_{2}^{r}=\sqrt{g(r)}$. The projections of the Ricci and Riemann tensors on the subspace orthogonal to $\Sigma$ are

$$
R_{i i}=-\frac{2 r_{-}}{r_{+}^{3}}, \quad R_{i j i j}=\frac{2 r_{+}-4 r_{-}}{r_{+}^{3}} .
$$

Since $R=0$ for the Reissner-Nordström metric, the entanglement entropy of a massless, minimally coupled, scalar field $(X=0)$ and of a conformally-coupled scalar field $X=-\frac{1}{6} R$ coincide [197],

$$
S_{\mathrm{RN}}=\frac{A(\Sigma)}{48 \pi \epsilon^{2}}+\frac{1}{90}\left(\frac{2 r_{+}-3 r_{-}}{r_{+}}\right) \ln \frac{r_{+}}{\epsilon}+s\left(\frac{r_{-}}{r_{+}}\right)
$$

where $A(\Sigma)=4 \pi r_{+}^{2}$ and $s\left(\frac{r_{-}}{r_{+}}\right)$represents the UV finite term. Since $s$ is dimensionless it may depend only on the ratio $\frac{r_{-}}{r_{+}}$of the parameters, which characterize the geometry of the black hole.

If the black hole geometry is characterized by just one dimensionful parameter, the UV finite term in Eq. (87) becomes an irrelevant constant. Let us consider two cases when this happens. 
The Schwarzschild black hole. In this case $r_{-}=0(q=0)$ and $r_{+}=2 m$ so that the entropy, found by Solodukhin [196], is

$$
S_{\mathrm{Sch}}=\frac{A(\Sigma)}{48 \pi \epsilon^{2}}+\frac{1}{45} \ln \frac{r_{+}}{\epsilon} .
$$

Historically, this was the first time when the subleading logarithmic term in entanglement entropy was computed. The leading term in this entropy is the same as in the Rindler space, when the actual black-hole spacetime is approximated by flat Rindler spacetime. This approximation is sometimes argued to be valid in the limit of infinite mass $M$. However, we see that, even in this limit, there always exists the logarithmic subleading term in the entropy of the black hole that was absent in the case of the Rindler horizon. The reason for this difference is purely topological. The Euler number of the black-hole spacetime is non-zero while it vanishes for the Rindler spacetime; the Euler number of the black-hole horizon (a sphere) is 2, while it is zero for the Rindler horizon (a plane).

The extreme charged black hole. The extreme geometry is obtained in the limit $r_{-} \rightarrow r_{+}$ $(q=m)$. The entropy of the extreme black hole is found to take the form [197]

$$
S_{\mathrm{ext}}=\frac{A(\Sigma)}{48 \pi \epsilon^{2}}-\frac{1}{90} \ln \frac{r_{+}}{\epsilon}
$$

Notice that we have omitted the irrelevant constants $s(0)$ and $s(1)$ in Eq. (88) and (89) respectively.

\subsubsection{The dilatonic charged black hole}

The metric of a dilatonic black hole, which has mass $m$, electric charge $q$ and magnetic charge $P$ takes the form [120]:

$$
d s^{2}=g(r) d \tau^{2}+g^{-1}(r) d r^{2}+R^{2}(r) d\left(d \theta^{2}+\sin ^{2} \theta d \phi^{2}\right)
$$

with the metric functions

$$
g(r)=\frac{\left(r-r_{+}\right)\left(r-r_{-}\right)}{R^{2}(r)}, \quad R^{2}(r)=r^{2}-D^{2},
$$

where $D$ is the dilaton charge, $D=\frac{P^{2}-q^{2}}{2 m}$. The outer and the inner horizons are defined by

$$
r_{ \pm}=m \pm \sqrt{m^{2}+D^{2}-P^{2}-q^{2}} .
$$

The entanglement entropy is defined for the outer horizon at $r=r_{+}$. The Ricci scalar of metric (90)

$$
R=-2 D^{2} \frac{\left(r-r_{+}\right)\left(r-r_{-}\right)}{\left(r^{2}-D^{2}\right)^{3}}
$$

vanishes at the outer horizon, $r=r_{+}$. Therefore, the entanglement entropy associated with the outer horizon is the same for a minimal scalar field $(X=0)$ and for a conformally-coupled scalar field $\left(X=-\frac{1}{6} R\right)$,

$$
S_{\text {dilaton }}=\frac{A_{\Sigma}}{48 \pi \epsilon^{2}}+\frac{1}{90}\left(\frac{3 r_{+}\left(r_{+}-r_{-}\right)}{\left(r_{+}^{2}-D^{2}\right)}-1\right) \log \frac{r_{+}}{\epsilon}+s\left(\frac{r_{-}}{r_{+}}, \frac{D}{r_{+}}\right),
$$

where $A_{\Sigma}=4 \pi\left(r_{+}^{2}-D^{2}\right)$ is the area of the outer horizon. 
It is instructive to consider the black hole with only electric charge (the magnetic charge $P=0$ in this case). This geometry is characterized by two parameters: $m$ and $q$. In this case one finds

$$
r_{+}=2 m-\frac{q^{2}}{2 m}, r_{-}=\frac{q^{2}}{2 m}, r_{+}^{2}-D^{2}=4 m\left(m-\frac{q^{2}}{2 m}\right)
$$

so that expression (93) takes the form

$$
S_{\text {dilaton }}=\frac{A_{\Sigma}}{48 \pi \epsilon^{2}}+\frac{1}{180}\left(1+3\left(1-\frac{q^{2}}{2 m^{2}}\right)\right) \ln \frac{r_{+}}{\epsilon}+s\left(\frac{q}{m}\right) .
$$

In the extremal limit, $2 m^{2}=q^{2}$, the area of the outer horizon vanishes, $A_{\Sigma}=0$, and the whole black-hole entropy is determined only by the logarithmically-divergent term ${ }^{5}$ (using a different brick-wall method a similar conclusion was reached in [114])

$$
S_{\text {ext-dil }}=\frac{1}{180} \log \frac{r_{+}}{\epsilon} .
$$

In this respect the extreme dilatonic black hole is similar to a two-dimensional black hole. Notice that Eq. (95) is positive as it should be since the entanglement entropy is, by definition, a positive quantity.

The calculation of the entanglement entropy of a static black hole is discussed in the following papers $[102,94,110,61,104,82,28,227,48,47,46,135,137,176,196,197,117,118,114,115,116]$.

\subsection{Entanglement Entropy of the Kerr-Newman black hole}

The geometry of the rotating black hole is more subtle than that of a static black hole: near the horizon the rotating spacetime is no longer a product of a horizon sphere $S_{2}$ and a two-dimensional disk. The other difficulty with applying the technique of the heat kernel to this case is that the Euclidean version of the geometry requires the rotation parameter to be complex. Nevertheless with some care these difficulties can be overcome and the entanglement entropy of a rotating black hole can be computed along the same lines as for a static black hole [170]. In this section we briefly review the results of Mann and Solodukhin [170].

\subsubsection{Euclidean geometry of Kerr-Newman black hole}

First we describe the Euclidean geometry in the near-horizon limit of the Kerr-Newmann black hole. The Euclidean Kerr-Newman metric can be written in the form

$$
d s_{E}^{2}=\frac{\hat{\rho}^{2}}{\hat{\Delta}} d r^{2}+\frac{\hat{\Delta} \hat{\rho}^{2}}{\left(r^{2}-\hat{a}^{2}\right)^{2}} \omega^{2}+\hat{\rho}^{2}\left(d \theta^{2}+\sin ^{2} \theta \tilde{\omega}^{2}\right),
$$

where the Euclidean time is $t=\imath \tau$ and the rotation and charge parameters have also been transformed $a=\imath \hat{a}, q=\imath \hat{q}$, so that the metric (96) is purely real. Here $\hat{\Delta}(r)=\left(r-\hat{r}_{+}\right)\left(r-\hat{r}_{-}\right)$, where $\hat{r}_{ \pm}=m \pm \sqrt{m^{2}+\hat{a}^{2}+\hat{q}^{2}}$, the quantities $\omega$ and $\tilde{\omega}$ take the form

$$
\omega=\frac{\left(r^{2}-\hat{a}^{2}\right)}{\hat{\rho}^{2}}\left(d \tau-\hat{a} \sin ^{2} \theta d \phi\right), \quad \tilde{\omega}=\frac{\left(r^{2}-\hat{a}^{2}\right)}{\hat{\rho}^{2}}\left(d \phi+\frac{\hat{a}}{\left(r^{2}-\hat{a}^{2}\right)} d \tau\right)
$$

with $\hat{\rho}^{2}=r^{2}-\hat{a}^{2} \cos ^{2} \theta$. This spacetime has a pair of orthogonal Killing vectors

$$
K=\partial_{\tau}-\frac{\hat{a}}{r^{2}-\hat{a}^{2}} \partial_{\phi}, \quad \tilde{K}=\hat{a} \sin ^{2} \theta \partial_{\tau}+\partial_{\phi},
$$

\footnotetext{
${ }^{5}$ Eqs. (93), (94) and (95) correct some errors in Eqs. (27) - (29) of [197].
} 
which are the respective analogs of the vectors $\partial_{\tau}$ and $\partial_{\phi}$ in the (Euclidean) Schwarzschild case. The horizon surface $\Sigma$ defined by $r=\hat{r}_{+}$is the stationary surface of the Killing vector $K$. Near this surface the metric (96) is approximately

$$
d s_{E}^{2}=d s_{\Sigma}^{2}+\hat{\rho}_{+}^{2} d s_{C_{2}}^{2}
$$

where $\hat{\rho}_{+}^{2}=\hat{r}_{+}^{2}-\hat{a}^{2} \cos ^{2} \theta$ and

$$
d s_{\Sigma}^{2}=\hat{\rho}_{+}^{2} d \theta^{2}+\frac{\left(\hat{r}_{+}^{2}-\hat{a}^{2}\right)^{2}}{\hat{\rho}_{+}^{2}} \sin ^{2} \theta d \psi^{2}
$$

is the metric on the horizon surface $\Sigma$ up to $O\left(x^{2}\right)$, where variable $x$ is defined by the relation $\left(r-\hat{r}_{+}\right)=\frac{\gamma x^{2}}{4}$ and $\gamma=2 \sqrt{m^{2}+\hat{a}^{2}+\hat{q}^{2}}$. The angle co-ordinate $\psi=\phi+\frac{\hat{a}}{\left(\hat{r}_{+}^{2}-\hat{a}^{2}\right)} \tau$ and is well defined on $\Sigma$. The metric $d s_{C_{2}}^{2}$ is that of a two-dimensional disk $C_{2}$

$$
d s_{C_{2}}^{2}=d x^{2}+\frac{\gamma^{2} x^{2}}{4 \hat{\rho}_{+}^{4}} d \chi^{2}
$$

attached to $\Sigma$ at a point $(\theta, \psi)$, where $\chi=\tau-\hat{a} \sin ^{2} \theta \phi$ is an angle co-ordinate on $C_{2}$.

Regularity of the metric near the horizon implies the identifications $\psi \leftrightarrow \psi+2 \pi$ and $\chi \leftrightarrow$ $\chi+4 \pi \gamma^{-1} \hat{\rho}_{+}^{2}$. For this latter condition to hold, independently of $\theta$ on the horizon, it is also necessary to identify $(\tau, \phi)$ with $\left(\tau+2 \pi \beta_{H}, \phi-2 \pi \Omega \beta_{H}\right)$, where $\Omega=\frac{\hat{a}}{\left(\hat{r}_{+}^{2}-\hat{a}^{2}\right)}$ is the (complex) angular velocity and $\beta_{H}=\left(\hat{r}_{+}^{2}-\hat{a}^{2}\right) / \sqrt{m^{2}+\hat{a}^{2}+\hat{q}^{2}}$. The identified points have the same coordinate $\psi$.

Therefore, near $\Sigma$ we have the following description of the Euclidean Kerr-Newman geometry: attached to every point $(\theta, \psi)$ of the horizon is a two-dimensional disk $C_{2}$ with coordinates $(x, \chi)$. The periodic identification of points on $C_{2}$ holds independently for different points on the horizon $\Sigma$, even though $\chi$ is not a global coordinate. As in the static case, there is an abelian isometry generated by the Killing vector $K$, whose fixed set is $\Sigma$. Locally we have $K=\partial_{\chi}$. The periodicity is in the direction of the vector $K$ and the resulting Euclidean space $E$ is a regular manifold.

Now consider closing the trajectory of $K$ with an arbitrary period $\beta \neq \beta_{H}$. This implies the identification $(\tau+2 \pi \beta, \phi-2 \pi \Omega \beta)$, and the metric on $C_{2}$ becomes

$$
d s_{C_{2, \alpha}}^{2}=d x^{2}+\alpha^{2} x^{2} d \bar{\chi}^{2},
$$

where $\chi=\beta \hat{\rho}_{+}^{2}\left(\hat{r}_{+}^{2}-\hat{a}^{2}\right)^{-1} \bar{\chi}$ is a new angular coordinate, with period $2 \pi$. This is the metric of a two dimensional cone with angular deficit $\delta=2 \pi(1-\alpha), \alpha \equiv \frac{\beta}{\beta_{H}}$. With this new identification the metric (96) now describes the Euclidean conical space $E_{\alpha}$ with singular surface $\Sigma$.

The difference of the Kerr-Newman metric from the static case considered above is that the Euclidean space near the bifurcation surface is not a direct product of the surface $\Sigma$ and twodimensional cone $C_{2, \alpha}$. Instead, it is a nontrivial foliation of $C_{2, \alpha}$ over $\Sigma$. However, this foliation shares certain common features with the static case. Namely, the invariants constructed from quadratic combinations of extrinsic curvature of $\Sigma$ vanish identically.

\subsubsection{Extrinsic curvature of the horizon}

In the case of a static black hole we have argued that the presence of an abelian isometry with horizon being the stationary point of the isometry guarantees that the extrinsic curvature identically vanishes on the horizon. In fact this is also true in the case of a rotating black hole. The role of the abelian isometry generated by the Killing vector $K$ is less evident in this case. That is why, in this subsection, following the analysis of [170], we explicitly evaluate the extrinsic curvature for 
the Kerr-Newman black hole and demonstrate that quadratic invariants, that can be constructed with the help of the extrinsic curvature, vanish on the horizon.

With respect to the Euclidean metric (96) we may define a pair of orthonormal vectors $\left\{n_{a}=\right.$ $\left.n_{a}^{\mu} \partial_{\mu}, \quad a=1,2\right\}$ :

$$
n_{1}^{r}=\sqrt{\frac{\hat{\Delta}}{\hat{\rho}^{2}}} ; \quad n_{2}^{\tau}=\frac{\left(r^{2}-\hat{a}^{2}\right)}{\sqrt{\hat{\Delta} \hat{\rho}^{2}}}, \quad n_{2}^{\phi}=\frac{-\hat{a}}{\sqrt{\hat{\Delta} \hat{\rho}^{2}}} .
$$

Covariantly, these are

$$
n_{r}^{1}=\sqrt{\frac{\hat{\rho}^{2}}{\hat{\Delta}}} ; \quad n_{\tau}^{2}=\sqrt{\frac{\hat{\Delta}}{\hat{\rho}^{2}}}, \quad n_{\phi}^{2}=-\sqrt{\frac{\hat{\Delta}}{\hat{\rho}^{2}}} \hat{a} \sin ^{2} \theta .
$$

The vectors $n^{1}$ and $n^{2}$ are normal to the horizon surface $\Sigma\left(\right.$ defined as $\left.r=r_{+}, \Delta\left(r=r_{+}\right)=0\right)$, which is a two-dimensional surface with induced metric $\gamma_{\mu \nu}=g_{\mu \nu}-n_{\mu}^{1} n_{\nu}^{1}-n_{\mu}^{2} n_{\nu}^{2}$. With respect to the normal vectors $n^{a}, a=1,2$ one defines the extrinsic curvatures of the surface $\Sigma: \kappa_{\mu \nu}^{a}=$ $-\gamma_{\mu}^{\alpha} \gamma_{\nu}^{\beta} \nabla_{\alpha} n_{\beta}^{a}$. The exact expression for the components of extrinsic curvature is given in [170]. The trace of the extrinsic curvature, $\kappa^{a}=\kappa_{\mu \nu}^{a} g^{\mu \nu}$,

$$
\kappa^{1}=-\frac{2 r}{\hat{\rho}^{2}} \sqrt{\frac{\hat{\Delta}}{\hat{\rho}^{2}}}, \quad \kappa^{2}=0
$$

vanishes when restricted to the horizon surface $\Sigma$ defined by condition $\hat{\Delta}\left(r=\hat{r}_{+}\right)=0$. Moreover, the quadratic combinations

$$
\kappa_{\mu \nu}^{1} \kappa_{1}^{\mu \nu}=\frac{2 r^{2} \hat{\Delta}}{\hat{\rho}^{6}}, \quad \kappa_{\mu \nu}^{2} \kappa_{2}^{\mu \nu}=\frac{2 \hat{a}^{2} \cos ^{2} \theta \hat{\Delta}}{\hat{\rho}^{6}}
$$

vanish on the horizon $\Sigma$. Consequently, we have $\kappa_{\mu \nu}^{a} \kappa^{a \mu \nu}=0$ on the horizon.

\subsubsection{Entropy}

Applying the conical singularity method to calculate the entanglement entropy of a rotating black hole we have to verify that i) the curvature singularity at the horizon of a stationary black hole behaves in the same way as in the static case and ii) there are no extra surface terms in the heat kernel expansion for the rotating black hole. The first point was explicitly checked in [170]: the curvature formulas (56) - (59) are still valid in the stationary case. Regarding the second point, it was shown by Dowker [70] that for a generic metric with conical singularity at some surface $\Sigma$ the only modification of the surface terms in the heat kernel expansion (70) are due to the extrinsic curvature of $\Sigma$. For example, the surface coefficient $a_{2}^{\Sigma}$ may be modified by integrals over $\Sigma$ of terms $\kappa^{a} \kappa_{a}$ and $\kappa_{\mu \nu}^{a} \kappa^{a \mu \nu}$. Since, as was shown in Section 3.10.2, these terms identically vanish for the Kerr-Newman metric there is no modification of the surface terms in this case. Thus, the expression for the entropy (82) remains unchanged in the case of a rotating black hole. The Ricci scalar for the Kerr-Newmann metric is zero, $R=0$. The integrals of the projections of Ricci and Riemann tensors over horizon surface are

$$
\begin{aligned}
\int_{\Sigma} R_{i j i j} & =8 \pi \frac{\left(\hat{r}_{+}^{2}+\hat{q}^{2}\right)}{\hat{r}_{+}^{2}}+4 \pi \frac{\hat{q}^{2}}{\hat{r}_{+}^{2}} \frac{\left(\hat{r}_{+}^{2}-\hat{a}^{2}\right)}{\hat{a} \hat{r}_{+}} \ln \left(\frac{\hat{r}_{+}+\hat{a}}{\hat{r}_{+}-\hat{a}}\right) \\
\int_{\Sigma} R_{i i} & =4 \pi \frac{\hat{q}^{2}}{\hat{r}_{+}^{2}}\left(1+\frac{\left(\hat{r}_{+}^{2}-\hat{a}^{2}\right)}{2 \hat{a} \hat{r}_{+}} \ln \left(\frac{\hat{r}_{+}+\hat{a}}{\hat{r}_{+}-\hat{a}}\right)\right)
\end{aligned}
$$


The analytic continuation of these expressions back to real values of the parameters $a$ and $q$ requires the substitution

$$
\begin{aligned}
& \hat{q}^{2}=-q^{2}, \quad \hat{a}^{2}=-a^{2}, \quad \hat{r}_{+}=r_{+} \\
& \frac{1}{\hat{a}} \ln \left(\frac{\hat{r}_{+}+\hat{a}}{\hat{r}_{+}-\hat{a}}\right)=\frac{2}{a} \tan ^{-1}\left(\frac{a}{r_{+}}\right) .
\end{aligned}
$$

With these identities the quantum entropy of the Kerr-Newman black hole reads [170]

$$
S_{\mathrm{KN}}=\frac{A(\Sigma)}{48 \pi \epsilon^{2}}+\frac{1}{45}\left(1-\frac{3 q^{2}}{4 r_{+}^{2}}\left(1+\frac{\left(r_{+}^{2}+a^{2}\right)}{a r_{+}} \tan ^{-1}\left(\frac{a}{r_{+}}\right)\right)\right) \ln \frac{1}{\epsilon},
$$

where $A(\Sigma)=4 \pi\left(r_{+}^{2}+a^{2}\right)$ is the area of the horizon $\Sigma$. In the limit $a \rightarrow 0$ this expression reduces to that of the Reissner-Nordström black hole (87). An interesting and still somewhat puzzling feature of this result is that, in the case of the Kerr black hole, described by the Kerr-Newman metric with vanishing electric charge $(q=0)$, the logarithmic term in the entropy does not depend on the rotation parameter $a$ and is the same as in the case of the Schwarzschild black hole. In particular for the extreme Kerr black hole $(q=0, m=a)$ one has

$$
S_{\text {Kerr }}=\frac{A(\Sigma)}{48 \pi \epsilon^{2}}+\frac{1}{45} \ln \frac{r_{+}}{\epsilon} .
$$

The entropy of the Kerr black hole in the brick-wall model was calculated in [45] and a result different from Eq. (109) was found. However, the subsequent study in [92] has confirmed Eq. (109).

\subsection{Entanglement entropy as one-loop quantum correction}

A natural point of view on the entanglement entropy of a black hole is that this entropy, as was suggested by Callan and Wilczek [33], is the first quantum correction to the Bekenstein-Hawking entropy $^{6}$. Indeed, the Bekenstein-Hawking entropy $S_{\mathrm{BH}}$ can be considered as classical, or tree-level, entropy. If we restore the presence of the Planck constant $\hbar$ the Bekenstein-Hawking entropy $S_{\mathrm{BH}}$ is proportional to $1 / \hbar$ while the entanglement entropy $S_{\text {ent }}$ is an $\hbar^{0}$ quantity. The total entropy of a black hole is then the sum

$$
S=S_{\mathrm{BH}}+S_{\mathrm{ent}}
$$

where all particles that exist in nature contribute to the entanglement entropy $S_{\text {ent }}$.

\subsection{The statement on the renormalization of the entropy}

As explained in Sections 3.9 and 3.10, the entanglement entropy is a UV divergent quantity. The other well-known quantity, which possesses UV divergences is the effective action. The standard way to handle the UV divergences in the action is to absorb them into a redefinition of the couplings, which appear in the gravitational action. In four dimensions the gravitational action should also include the terms quadratic in the Riemann curvature. The renormalization procedure is well studied and is described in textbooks (see for instance [22]). The idea now is that exactly the same procedure renormalizes the UV divergences in the entropy. In order to demonstrate this statement, consider a minimally-coupled scalar field. For simplicity suppose that the mass of

\footnotetext{
6 This statement should be taken with some care. Entanglement entropy is a small correction compared to the Bekenstein-Hawking entropy if the UV cutoff $1 / \epsilon$ is, for example, on the order of a few GeV (energy scale of the standard model). However, the two entropies are of the same order if the cutoff is at the Planck scale. I thank 't Hooft for his comments on this point.
} 
the field vanishes. The bare (tree-level) gravitational action in four dimensions is the sum of the Einstein-Hilbert term and all possible combinations quadratic in the Riemann curvature,

$$
W_{\mathrm{gr}}=\int \sqrt{g} d^{4} x\left(-\frac{1}{16 \pi G_{B}}\left(R+2 \Lambda_{B}\right)+c_{1, B} R^{2}+c_{2, B} R_{\mu \nu}^{2}+c_{3, B} R_{\mu \nu \alpha \beta}^{2}\right),
$$

where $G_{B}, \Lambda_{B}, c_{1, B}, c_{2, B}, c_{3, B}$ are the bare coupling constants in the gravitational action.

The UV divergences of the gravitational action are computed by the heat kernel method using the small $s$ expansion (69). For a minimal massless field ( $X=0$ in the scalar field equation) one finds

$$
W_{\mathrm{div}}(\epsilon)=-\frac{1}{64 \pi^{2} \epsilon^{4}} \int_{E} 1-\frac{1}{192 \pi^{2} \epsilon^{2}} \int_{E} R+\frac{1}{16 \pi^{2}} \int_{E}\left(\frac{1}{180} R_{\alpha \beta \mu \nu}^{2}-\frac{1}{180} R_{\alpha \beta}^{2}+\frac{1}{72} R^{2}\right) \ln \epsilon(.113)
$$

These divergences are removed by standard renormalization of the gravitational couplings in the bare gravitational action

$$
W_{\mathrm{gr}}\left(G_{B}, c_{i, B}, \Lambda_{B}\right)+W_{\mathrm{div}}(\epsilon)=W_{\mathrm{gr}}\left(G_{\mathrm{ren}}, c_{i, \mathrm{ren}}, \Lambda_{\mathrm{ren}}\right),
$$

where $G_{\text {ren }}$ and $c_{i, r e n}$ are the renormalized couplings expressed in terms of the bare ones and the UV parameter $\epsilon$;

$$
\begin{array}{rlrl}
\frac{1}{G_{\text {ren }}}=\frac{1}{G_{B}}+\frac{1}{12 \pi \epsilon^{2}}, & c_{1, \text { ren }} & =c_{1, B}+\frac{1}{32 \pi^{2}} \frac{1}{36} \ln \epsilon, \\
c_{2, \text { ren }}=c_{2, B}-\frac{1}{32 \pi^{2}} \frac{1}{90} \ln \epsilon, & c_{3, \text { ren }}=c_{3, B}+\frac{1}{32 \pi^{2}} \frac{1}{90} \ln \epsilon .
\end{array}
$$

The tree-level entropy can be obtained by means of the same replica trick, considered in Sections 3.8, 3.9 and 3.10, upon introduction of the conical singularity with a small angle deficit $2 \pi(1-\alpha), S\left(G_{B}, c_{i, B}\right)=\left(\alpha \partial_{\alpha}-1\right) W_{\mathrm{gr}}(\alpha)$. The conical singularity at the horizon $\Sigma$ manifests itself in that a part of the Riemann tensor for such a manifold $E_{\alpha}$ behaves as a distribution having support on the surface $\Sigma$. Using formulas (56)-(59) one finds for the tree-level entropy

$$
S\left(G_{B}, c_{i, B}\right)=\frac{1}{4 G_{B}} A(\Sigma)-\int_{\Sigma}\left(8 \pi c_{1, B} R+4 \pi c_{2, B} R_{i i}+8 \pi c_{3, B} R_{i j i j}\right) .
$$

Thus, the Bekenstein-Hawking entropy $S=\frac{1}{4 G} A(\Sigma)$ is modified due to the presence of $R^{2}$-terms in the action (112). It should be noted that Eq. (116) exactly coincides with the entropy computed by the Noether charge method of Wald [221, 144] (the relation between Wald's method and the method of conical singularity is discussed in [140]).

The UV divergent part of the entanglement entropy of a black hole has already been calculated, see Eq. (82). For a minimal massless scalar, one has

$$
S_{\mathrm{div}}=\frac{A(\Sigma)}{48 \pi \epsilon^{2}}-\frac{1}{144 \pi} \int_{\Sigma}\left(R-\frac{1}{5}\left(R_{i i}-2 R_{i j i j}\right)\right) \ln \epsilon .
$$

The main point now is that the sum of the UV divergent part (117) of the entanglement entropy and the tree-level entropy (116)

$$
S\left(G_{B}, c_{i, B}\right)+S_{\mathrm{div}}(\epsilon)=S\left(G_{\mathrm{ren}}, c_{i, \mathrm{ren}}\right)
$$

takes again the tree-level form (116) if expressed in terms of the renormalized coupling constants $G_{\text {ren }}, c_{i \text {,ren }}$ defined in Eq. (115). Thus, the UV divergences in entanglement entropy can be handled 
by the standard renormalization of the gravitational couplings, so that no separate renormalization procedure for the entropy is required.

It should be noted that the proof of the renormalization statement is based on a nice property of the heat kernel coefficients $a_{n}$ (68) on space with conical singularity. Namely, up to $(1-\alpha)^{2}$ terms the exact coefficient $a_{n}=a_{n}^{\text {reg }}+a_{n}^{\Sigma}$ on conical space $E_{\alpha}$ is equal to the regular volume coefficient $a_{n}^{\text {reg }}$ expressed in terms of the complete curvature, regular part plus a delta-like contribution, using relations $(55)$

$$
a_{n}\left(E_{\alpha}\right)=a_{n}^{\mathrm{reg}}(\bar{R})+a_{N}^{\Sigma}=a_{n}^{\mathrm{reg}}\left(\bar{R}+R^{\mathrm{sing}}\right)+O\left((1-\alpha)^{2}\right) .
$$

The terms quadratic in $R^{\text {sing }}$ are not well defined. However, these terms are proportional to $(1-\alpha)^{2}$ and do not affect the entropy calculation. Thus, neglecting terms of order $(1-\alpha)^{2}$ in the calculation of entropy, the renormalization of entropy (118) directly follows from the renormalization of the effective action (114).

That the leading $1 / \epsilon^{2}$ divergence in the entropy can be handled by the standard renormalization of Newton's constant $G$ has been suggested by Susskind and Uglum [213] and by Jacobson [141]. That one also has to renormalize the higher curvature couplings in the gravitational action in order to remove all divergences in the entropy of the Schwarzschild black hole was suggested by Solodukhin [196]. For a generic static black hole the renormalization statement was proven by Fursaev and Solodukhin in [112]. In a different approach based on 't Hooft's "brick-wall model" the renormalization was verified for the Reissner-Nordström black hole by Demers, Lafrance and Myers [62]. For the rotating black hole described by the Kerr-Newman metric the renormalization of the entropy was demonstrated by Mann and Solodukhin [170]. The non-equilibrium aspect (as defining the rate in a semiclassical decay of hot flat space by black hole nucleation) of the black hole entropy and the renormalization was discussed by Barbon and Emparan [12].

\subsection{Renormalization in theories with a modified propagator}

Let us comment briefly on the behavior of the entropy in theories described by a wave operator $\mathcal{D}=F\left(-\nabla^{2}\right)$, which is a function of the standard Laplace operator $\nabla^{2}$. In flat space this was analyzed in Section 2.12. As is shown in [184] there is a precise relation between the small $s$ expansion of the heat kernel of operator $F\left(-\nabla^{2}\right)$ and that of the Laplace operator $-\nabla^{2}$. The latter heat kernel has the standard decomposition

$$
\operatorname{Tr} e^{s \nabla^{2}}=\frac{1}{(4 \pi)^{d / 2}} \sum_{n=0} a_{n} s^{n-d / 2} .
$$

The heat kernel of operator $F\left(-\nabla^{2}\right)$ then has the decomposition [184]

$$
\operatorname{Tr} e^{-s F\left(-\nabla^{2}\right)}=\frac{1}{(4 \pi)^{d / 2}} \sum_{n=0} a_{n} \mathcal{T}_{n}(s),
$$

where

$$
\mathcal{T}_{n}(s)=\left\{\begin{array}{ll}
P_{d-2 n}(s) & n<d / 2 \\
\left.\left(-\partial_{q}\right)^{n-d / 2} e^{-s F(q)}\right|_{q=0} & n \geq d / 2
\end{array} .\right.
$$

In even dimension $d$ the term $\mathcal{T}_{d / 2}(s)=1$. This decomposition is valid both for regular manifolds and manifolds with a conical singularity. If a conical singularity is present, the coefficients $a_{n}$ have the standard decomposition into regular $a_{n}^{\text {reg }}$ and surface $a_{n}^{\Sigma}$ parts as in Eq. (68). The surface term for $n=1$ is just the area of the surface $\Sigma$, while the surface terms with $n \geq 2$ contain surface integrals of $(n-1)$-th power of the Riemann curvature. Thus, Eq. (121) is a decomposition in powers of the curvature of the spacetime. 
The functions $P_{n}$ are defined in Eq. (40). In particular, if $F(q)=q^{k}(k>0)$ one finds that

$$
P_{n}(s)=s^{-\frac{n}{2 k}} \frac{\Gamma\left(\frac{n}{2 k}\right)}{\Gamma\left(\frac{n}{2}\right) k} .
$$

The terms with $n \leq d / 2$ in decomposition (121) produce the UV divergent terms in the effective action and entropy. The term $n=d / 2$ gives rise to the logarithmic UV divergence. In $d$ dimensions the area term in the entropy is the same as in flat spacetime (see Eq.(41)). In four dimensions $(d=4)$ the UV divergent terms in the entropy are

$$
S=\frac{A(\Sigma)}{48 \pi} \int_{\epsilon^{2}}^{\infty} \frac{d s}{s} P_{2}(s)-\frac{1}{144 \pi} \int_{\Sigma}\left(R-\frac{1}{5}\left(R_{i i}-2 R_{i j i j}\right)\right) \ln \epsilon .
$$

We note that an additional contribution to the logarithmic term may come from the first term in Eq. (124) (for instance, this is so for the Laplace operator modified by the mass term, $F(q)=$ $\left.q+m^{2}\right)$

In the theory with operator $F\left(-\nabla^{2}\right)$ Newton's constant is renormalized as [184]

$$
\frac{1}{G_{\mathrm{ren}}}=\frac{1}{G_{B}}+\frac{1}{12 \pi} \int_{\epsilon^{2}}^{\infty} \frac{d s}{s} P_{2}(s) .
$$

while the higher curvature couplings $c_{i}, i=1,2,3$ in the effective action are renormalized in the same way as in Eq. (115). The renormalization of $G$ and $\left\{c_{i}\right\}$ then makes both the effective action and the entropy finite in the exact same way as in the case of the Laplace operator $-\nabla^{2}$. Thus, the renormalization statement generalizes to the theories with modified wave operator $F\left(-\nabla^{2}\right)$.

\subsection{Area law: generalization to higher spin fields}

In this section, we will focus only on the leading UV divergent term, proportional to the area of the horizon. The proportionality of the entanglement entropy to the area is known as the "area law". As we have discussed already for the case of a scalar field, this term in the entanglement entropy of a black hole is the same as in flat spacetime. In flat Minkowski spacetime, for a field of spin $s$, massive or massless, including the gauge fields, the calculation of entanglement entropy effectively reduces to the scalar field calculation, provided the number of scalar fields is equal to the number of physical degrees of freedom of the spin- $s$ field in question. The contribution of fermions comes with the weight $1 / 2$. Thus, we can immediately write down the general expression for the entanglement entropy of a quantum field of spin $s$ in $d$ dimensions,

$$
S_{(s, d)}=\frac{\mathcal{D}_{s}(d)}{6(d-2)(4 \pi)^{\frac{d-2}{2}}} \frac{A(\Sigma)}{\epsilon^{d-2}},
$$

where $\mathcal{D}_{s}(d)$ is (with weight $1 / 2$ for fermionic fields) the number of physical (on-shell) degrees of freedom of a particle of spin $s$ in $d$ dimensions. For gauge fields this assumes gauge fixing. In particular one has

$$
\mathcal{D}_{1 / 2}(d)=\frac{2^{[d / 2]}}{2}
$$

for Dirac fermions,

$$
\mathcal{D}_{1}(d)=(d-2) \cdot \operatorname{dim} G
$$


for the gauge vector fields (including the contribution of ghosts), where $\operatorname{dim} G$ is the $\operatorname{dimension}$ of the gauge group,

$$
\mathcal{D}_{3 / 2}(d)=(d-2) \frac{2^{[d / 2]}}{2}
$$

for the Rarita-Schwinger particles of spin 3/2 with gauge symmetry (gravitinos), and

$$
\mathcal{D}_{2}(d)=\frac{d(d-3)}{2}
$$

for massless spin-2 particles (gravitons).

The result for Dirac fermions was first obtained by Larsen and Wilczek [162, 163] and later in a paper by Kabat [150]. The contribution of the gauge fields to the entropy was derived by Kabat [150]. The entropy of the Rarita-Schwinger spin-3/2 particle and of a massless graviton was analyzed by Fursaev and Miele ${ }^{7}[110]$.

\subsection{Renormalization of entropy due to fields of different spin}

The effective action of a field of spin $s$ can be written as

$$
W_{(s)}=\frac{(-)^{2 s}}{2} \int_{\epsilon^{2}}^{\infty} \frac{d s}{s} \operatorname{Tr} e^{-s \Delta^{(s)}} .
$$

The second-order covariant operators acting on the spin-s field can be represented in the following general form

$$
\Delta^{(s)}=-\nabla^{2}+X^{(s)},
$$

where the matrices $X_{(s)}$ depend on the chosen representation of the quantum field and are linear in the Riemann tensor. Here are some examples [43, 44]

$$
\begin{aligned}
& X^{(0)}=\xi R, \quad X_{A B}^{(1 / 2)}=\frac{1}{4} R \delta_{A B}, \quad X_{\mu \nu}^{(1)}= \pm R_{\mu \nu} \\
& X_{A B, \mu \nu}^{(3 / 2)}=\frac{1}{4} R \delta_{A B} g_{\mu \nu}-\frac{1}{2} R_{\mu \nu \alpha \beta}\left(\gamma^{\alpha} \gamma^{\beta}\right)_{A B}, \\
& X_{\mu \nu, \alpha \beta}^{(2)}=\frac{1}{2} R\left(g_{\mu \alpha} g_{\nu \beta}+g_{\mu \beta} g_{\nu \alpha}\right)-R_{\alpha \mu} g_{\beta \nu}-R_{\beta \nu} g_{\alpha \mu}-R_{\mu \alpha \nu \beta}-R_{\nu \alpha \mu \beta},
\end{aligned}
$$

where $\gamma_{A B}^{\alpha}$ are gamma-matrices. The coefficient $a_{1}^{\text {reg }}$ in the small $s$ expansion $(67)-(69)$ of the heat kernel of operator (132) has the general form

$$
a_{1}^{(s)}=\int_{E}\left(\frac{D_{s}(d)}{6} R-\operatorname{Tr} X^{(s)}\right),
$$

where $D_{s}(d)$ is the dimension of the representation of spin $s$,

$$
\begin{aligned}
& D_{s=0}=1, \quad D_{s=1 / 2}=2^{[d / 2]}, \quad D_{s=1}=d, \\
& D_{s=3 / 2}=d 2^{[d / 2]}, \quad D_{s=2}=\frac{(d-1)(d+2)}{2} .
\end{aligned}
$$

$D_{s}(d)$ can be interpreted as the number of off-shell degrees of freedom.

Let us consider some particular cases.

\footnotetext{
7 Among other things the authors of [110] observe certain non-smooth behavior of the heat kernel coefficients for the spin-3/2 and spin-2 fields in the limit of vanishing angle deficit.
} 
Dirac fermions $(s=\mathbf{1} / \mathbf{2})$. The partition function for Dirac fermions is $Z_{1 / 2}=\operatorname{det}^{1 / 2} \Delta^{(1 / 2)}$. In this case $\operatorname{Tr} X_{(1 / 2)}=\frac{1}{4} 2^{[d / 2]} R$ and hence

$$
a_{1}^{(s=1 / 2)}=-\frac{\mathcal{D}_{1 / 2}}{6} \int_{E} R
$$

where $\mathcal{D}_{1 / 2}$ was introduced in Eq. (127). We note that the negative sign in Eq. (136) in combination with the negative sign for fermions in the effective action (131) gives the total positive contribution to Newton's constant. The renormalization of Newton's constant due to Dirac fermions is

$$
\frac{1}{4 G_{\mathrm{ren}}}=\frac{1}{4 G}+\frac{1}{(4 \pi)^{\frac{d-2}{2}}(d-2)} \frac{\mathcal{D}_{1 / 2}}{6} \frac{1}{\epsilon^{d-2}} .
$$

Comparison of this equation with the UV divergence of entropy (126) for spin-1/2 shows that the leading UV divergence in the entropy of spin-1/2 field is handled by the renormalization of Newton's constant in the same manner as it was for a scalar field.

The Rarita-Schwinger field $(s=\mathbf{3} / \mathbf{2})$. The partition function, including gauge fixing and the Faddeev-Popov ghost contribution, in this case, is

$$
Z_{3 / 2}=\operatorname{det}^{1 / 2} \Delta^{(3 / 2)} \operatorname{det}^{-1} \Delta^{(1 / 2)}
$$

so that the appropriate heat kernel coefficient is

$$
a_{1}=a_{1}^{(3 / 2)}-2 a^{(1 / 2)}=-\frac{\mathcal{D}_{3 / 2}}{6} \int_{E} R,
$$

where $\mathcal{D}_{3 / 2}$ is introduced in Eq. (129). The renormalization of Newton's constant

$$
\frac{1}{4 G_{\text {ren }}}=\frac{1}{4 G}+\frac{1}{(4 \pi)^{\frac{d-2}{2}}(d-2)} \frac{\mathcal{D}_{3 / 2}}{6} \frac{1}{\epsilon^{d-2}}
$$

then, similarly to the case of Dirac fermions, automatically renormalizes the entanglement entropy (126).

However, this property, does not hold for all fields. The main role in the mismatch between the UV divergences in the entanglement entropy and in Newton's constant is played by the non-minimal coupling terms $X^{(s)}$, which appear in the field operators (132).

\subsection{The puzzle of non-minimal coupling}

The simplest case to consider is that of a

Non-minimally coupled scalar field. In this case, one has $\operatorname{Tr} X^{(0)}=\xi R$, where $\xi$ is the parameter of non-minimal coupling. The renormalization of Newton's constant

$$
\frac{1}{4 G_{\mathrm{ren}}}=\frac{1}{4 G}+\frac{1}{(4 \pi)^{\frac{d-2}{2}}(d-2)}\left(\frac{1}{6}-\xi\right) \frac{1}{\epsilon^{d-2}}
$$

is modified due to the presence of the non-minimal coupling $\xi$ in the scalar field operator. At the same time, the entropy calculation on a Ricci flat background $(\bar{R}=0)$ is not affected by the non-minimal coupling since the field operator for this background is identical to the minimal one. This simple reasoning shows that the area law in the case of a non-minimally coupled scalar field 
is the same as in the case of the minimal scalar field (81). Clearly, there is a mismatch between the renormalization of Newton's constant and the renormalization of the entanglement entropy. One concludes that, in the presence of non-minimal coupling, when the Riemann tensor appears explicitly in the action of the quantum field, the UV divergence of the entanglement entropy cannot be handled by the standard renormalization of Newton's constant. The mismatch in the entropy is

$$
S_{\xi}^{\text {non-min }}=\frac{(-\xi)}{(d-2)(4 \pi)^{\frac{d-2}{2}}} \frac{A(\Sigma)}{\epsilon^{d-2}} .
$$

It is an important fact that there is no known way to give a statistical meaning to this entropy. Moreover, (142) does not have a definite sign and may become negative if $\xi$ is positive. In some respects, this term is similar to the classical Bekenstein-Hawking entropy: both entropies, at least in the framework of the conventional field theory, do not have a well-defined statistical meaning. There is a hope that in string theory the terms similar to Eq. (142) may acquire a better meaning. However, this question is still open.

We should note that on a space with a conical singularity one can consider the Ricci scalar in the non-minimal scalar operator as the complete curvature including the $\delta$-like singular term as in Eq. (55). Then, the differential operator $-\left(\nabla^{2}+\xi R\right)$ contains a delta-like potential concentrated on the horizon surface $\Sigma$. The presence of this potential modifies the surface terms in the heat kernel in such a way that [198]

$$
a_{1}^{\Sigma}(\xi)=a_{1}^{\Sigma}(\xi=0)-4 \pi \xi(1-\alpha) \int_{\Sigma} 1+O(1-\alpha)^{2}
$$

where $a_{1}^{\Sigma}(\xi=0)$ is the surface term $a_{1}^{\Sigma}(70)$ without the non-minimal coupling and the term $O(1-\alpha)^{2}$ is ill defined (something like $\left.\delta^{2}(0)\right)$. However, it does not affect the entropy calculation. If we now apply the replica trick and calculate the entropy corresponding to the theory with the heat kernel with the surface term (143) we get that $[198,163,16]$

$$
S_{\mathrm{div}}=\frac{1}{(d-2)(4 \pi)^{\frac{d-2}{2}} \epsilon^{d-2}}\left(\frac{1}{6}-\xi\right) A(\Sigma) .
$$

This divergence takes a form consistent with the UV divergence of Newton'constant (141). However, we cannot interpret this entropy as a contribution to the entanglement entropy since the presence of the delta-like potential in the Euclidean field operator is not motivated from the point of view of the original Lorentzian theory, for which the entanglement entropy is calculated. Moreover, Eq. (144) is not positive if $\xi>1 / 6$, while the entanglement entropy is supposed to be a positive quantity.

Similar features are shared by other non-minimally-coupled fields.

Abelian vector field. After gauge fixing, the partition function of an abelian gauge field is

$$
Z=\operatorname{det}^{-1 / 2} \Delta_{+}^{(1)} \cdot \operatorname{det} \Delta^{(0)},
$$

where $\Delta_{+}^{(1)}$ is operator defined in Eq. (132) with sign + in the matrix $X_{\mu \nu}^{(1)}(133)$. For the effective action $W_{\text {eff }}=-\ln Z$ we find,

$$
W_{\mathrm{eff}}=-\frac{1}{2} \int_{\epsilon^{2}}^{\infty} \frac{d s}{s} \frac{1}{(4 \pi s)^{d / 2}}\left(\int_{E} 1+a_{1} s+. .\right),
$$


where

$$
a_{1}=a_{1}^{(s=1)}-2 a_{1}^{(s=0)}=\left(\frac{\mathcal{D}_{1}(d)}{6}-1\right) \int_{E} R,
$$

and $\mathcal{D}_{1}(d)=d-2$ is the number of on-shell degrees of freedom of the abelian vector field. The renormalization of Newton's constant is

$$
\frac{1}{4 G_{\mathrm{ren}}}=\frac{1}{4 G}+\frac{1}{(4 \pi)^{\frac{d-2}{2}}(d-2)}\left(\frac{\mathcal{D}_{1}(d)}{6}-1\right) \frac{1}{\epsilon^{d-2}} .
$$

Comparison with Eq. (126) shows that there is again a mismatch between the UV divergences in the entropy and in Newton's constant. This mismatch stems from the non-minimal term $X_{\mu \nu}^{(1)}$ in the Laplace type field operator for the vector field.

Massless graviton. The partition function of a massless graviton in $d$ dimensions, after gauge fixing and adding the Faddeev-Popov ghost contribution, is

$$
Z=\operatorname{det}^{-1 / 2} \Delta^{(2)} \cdot \operatorname{det} \Delta_{-}^{(1)} \cdot \operatorname{det}^{-1 / 2} \Delta^{(0)},
$$

where $\Delta_{-}^{(1)}$ is operator defined in Eq. (132) with sign - in the matrix $X_{\mu \nu}^{(1)}(133)$. The operator $\Delta^{(2)}$ governs the dynamics of the tensor perturbations, which satisfy the condition $\nabla^{\mu}\left(h_{\mu \nu}-\frac{1}{2} g_{\mu \nu} h\right)=0$. The operator $\Delta^{(0)}$ is due to the contribution of the conformal mode, while the determinant of operator $\Delta_{-}^{(1)}$ is due to the Faddeev-Popov ghosts. Hence, one has in this case that

$$
a_{1}=a_{1}^{s=2}-2 a_{1}^{(s=1)}+a_{1}^{(s=0)}=\left(\frac{\mathcal{D}_{2}(d)}{6}-c(d)\right) \int_{E} R, \quad c(d)=\frac{d^{2}-d+4}{2},
$$

where $\mathcal{D}_{2}(d)=\frac{d(d-3)}{2}$ is the number of on-shell degrees of freedom of a massless spin-2 particle. The renormalization of Newton's constant is

$$
\frac{1}{4 G_{\mathrm{ren}}}=\frac{1}{4 G}+\frac{1}{(4 \pi)^{\frac{d-2}{2}}(d-2)}\left(\frac{\mathcal{D}_{2}(d)}{6}-c(d)\right) \frac{1}{\epsilon^{d-2}} .
$$

Again, we observe the mismatch between the UV divergent terms in the entropy (126) and in Newton's constant, this time due to the graviton.

To summarize, the UV divergences in the entanglement entropy of minimally-coupled scalars and fermions are properly renormalized by the redefinition of Newton's constant. It happens that each minimally-coupled field (no matter bosonic or fermionic) contributes positively to Newton's constant and positively to the entropy of the black hole. The contributions to both quantities come proportionally, which allows the simultaneous renormalization of both quantities. The mismatch between the UV divergences in the entropy and in Newton's constant appears for gauge bosons: the abelian (and non-abelian) vector fields and gravitons. The source of the mismatch are those non-minimal terms $X^{(s)}$ in the field operator, which contribute negatively to Newton's constant and do not make any contribution to the entanglement entropy of the black hole. At the moment of writing of this review the appropriate treatment of the entropy of non-minimally-coupled fields is not yet available.

\subsection{Comments on the entropy of interacting fields}

So far we have considered free fields in a fixed gravitational (black hole) background. The interaction can be included by adding a potential term $\int d^{d} x V(\psi)$ to the classical action, here 
$\psi=\left\{\psi^{i}, i=1, \ldots, N\right\}$ is the set of fields in question. In the one-loop approximation, one splits $\psi=\psi_{c}+\psi_{q}$, where $\psi_{c}$ is the classical background field and $\psi_{q}$ is the quantum field. The integration over $\psi_{q}$ then reduces to the calculation of the functional determinant of operator $\mathcal{D}+M^{2}\left(\psi_{c}\right)$, where $M_{i j}^{2}=\partial_{i j}^{2} V\left(\psi_{c}\right)$. The fields $\psi_{c}$ representing the classical background are, in general, functions on the curved spacetime. In some cases these fields are constants that minimize the potential $V(\psi)$. The matrix $M_{i j}(x)$ plays the role of an $x$-dependent mass matrix. In the approximation, when one can neglect the derivatives of the matrix $M$, the heat kernel of operator $\mathcal{D}+M^{2}$ is presented as the product $\operatorname{Tr} e^{-s \mathcal{D}}$. Tr $e^{-s M^{2}}$. Using the already calculated trace of the heat kernel $\operatorname{Tr} e^{-s \mathcal{D}}$ on space with a conical singularity one obtains at one-loop the entanglement entropy of the interacting fields. In $d$ dimensions one obtains [205] (see also [175] for a related discussion)

$$
S_{(d)}=\frac{1}{12(4 \pi)^{\frac{d-2}{2}}} \int_{\Sigma} \operatorname{Tr}\left[M^{d-2} \Gamma\left(1-\frac{d}{2}, M^{2} \epsilon^{2}\right)\right],
$$

where we used that $\int_{\epsilon^{2}}^{\infty} s^{-d / 2} e^{-M^{2} s}=\Gamma\left(1-\frac{d}{2}, M^{2} \epsilon^{2}\right)$. Using the asymptotic behavior $\Gamma(-\alpha, x)=$ $\alpha^{-1} x^{-\alpha}+\ldots$, we find that the leading UV divergence of the entropy (152) is again (multiplied by $N)$ (81) and is thus not affected by the presence of the interaction in the action. However, the interaction shows up in the sub-leading UV divergent and the UV finite terms. For instance, in four dimensions on a flat background we find

$$
S=\frac{A(\Sigma)}{48 \pi} \frac{N}{\epsilon^{2}}+\frac{1}{48 \pi} \int_{\Sigma}\left((\gamma-1) \operatorname{Tr} M^{2}+\operatorname{Tr} M^{2} \ln \left(\epsilon^{2} M^{2}\right)\right) .
$$

We see that the leading UV divergent term proportional to the area is not modified by the presence of the self-interaction. The mass matrix $M^{2}$ is a function of the background field $\psi_{c}$. Thus, the result (153) indicates that at tree-level the entropy should contain terms additional to those of the standard area law, which depend on the value of field $\psi$ at the horizon. In order to illustrate this point consider a $\psi^{4}$ model of a single field (a two-dimensional model of this type was considered in [151], in four dimensions the role of self-interaction was discussed in [46])

$$
W[\psi]=\frac{1}{2} \int d^{4} x \sqrt{g}\left((\nabla \psi)^{2}+\xi R \psi^{2}+\frac{\lambda}{6} \psi^{4}\right),
$$

where we included the term with the non-minimal coupling. In fact, if we had not included this term, it would have been generated by the quantum corrections due to the self-interaction of the field $\psi^{4}$. This is a well-known fact, established in [182]. The renormalized non-minimal coupling in the model (154) is

$$
\xi_{\text {ren }}=\xi-\frac{\lambda}{8 \pi^{2}}\left(\frac{1}{6}-\xi\right) \ln \epsilon
$$

where we omit the terms of higher order in $\lambda$. Splitting the field $\psi$ into classical and quantum parts in Eq. (154) we find $M^{2}=\xi R+\lambda \psi_{c}^{2}$. Suppose for simplicity that the background metric is flat. Then to leading order in $\lambda$ the entanglement entropy (omitting the UV finite terms) is

$$
S_{\text {div }}=\frac{A}{48 \pi \epsilon^{2}}+\frac{\lambda}{24 \pi} \int_{\Sigma} \psi_{c}^{2} \ln \epsilon .
$$

This entropy should be considered as a quantum correction to the tree-level entropy

$$
S_{\text {tree }}=\frac{A}{4 G_{N}}-2 \pi \xi \int_{\Sigma} \psi_{c}^{2}
$$

which follows from the action $W_{\mathrm{gr}}+W[\psi]$. We see that the logarithmic divergences in Eqs. (156) and (155) agree if $\xi=0$. On the other hand, the renormalization of $\xi$ (155) does not make the total entropy $S_{\text {tree }}+S_{\text {div }}$ completely UV finite. This is yet another manifestation of the puzzling behavior of non-minimal coupling. 


\section{Other Related Methods}

\subsection{Euclidean path integral and thermodynamic entropy}

In 1977, Gibbons and Hawking [119] developed a method based on the Euclidean path integral for studying the thermodynamics of black holes. In this method one obtains what may be called a thermodynamic entropy. One deals with metrics, which satisfy the gravitational field equations, and thus avoid the appearance of metrics with conical singularities. The entanglement entropy, on the other hand, has a well-defined statistical meaning. In ordinary systems, the thermodynamic entropy and the statistical (microscopic) entropies coincide. For black holes the exact relation between the two entropies can be seen from the following reasoning ${ }^{8}$ [198].

Consider a gravitationally coupled system (gravity plus quantum matter fields) at some arbitrary temperature $T=(\beta)^{-1}$. A standard way to describe a thermal state of a field system is to use an Euclidean path integral over all fields in question defined on manifold with periodicity $2 \pi \beta$ along the time-like Killing vector. Suppose that it is a priori known that the system includes a black hole. Thus, there exists a surface $\Sigma$ (horizon), which is a fixed point of the isometry generated by the killing vector. This imposes an extra condition on the possible class of metrics in the path integral. The other condition to be imposed on metrics in the path integral is the asymptotic behavior at infinity: provided the mass $M$ and the electric charge $Q$ of the gravitational configuration are fixed, one has to specify the fall-off of the metrics for large values of $r$. Thus, the Euclidean path integral is

$$
Z(\beta, M, Q)=\int \mathcal{D} g_{\mu \nu} \int \mathcal{D} \psi e^{-W_{\mathrm{gr}}[g]+W_{\mathrm{mat}}[\psi, g]},
$$

where the integral is taken over $\beta$-periodic fields $\psi\left(\tau, x^{i}\right)=\psi(\tau+\beta, x)$ and over metrics, which satisfy the following conditions:

i) $g_{\mu \nu}$ possesses an abelian isometry with respect to the Killing vector $\partial_{\tau}$;

ii) there exists a surface $\Sigma$ (horizon) where the Killing vector $\partial_{\tau}$ becomes null;

iii) asymptotic fall-off of metric $g_{\mu \nu}$ at large values of radial coordinate $r$ is fixed by the mass $M$ and electric charge $Q$ of the configuration.

Since the inverse temperature $\beta$ and mass $M$ in the path integral are two independent parameters, the path integral (158) is mostly over metrics, which have a conical singularity at the surface $\Sigma$. The integration in Eq. (158) can be done in two steps. First, one computes the integral over matter fields $\psi$ on the background of a metric, which satisfies conditions i), ii) and iii). The result of this integration is the quantity (15) used in the computation of the entanglement entropy,

$$
\int \mathcal{D} \psi e^{-W_{\text {mat }}[\psi, g]}=e^{-W[\beta, g]}
$$

Semiclassically, the functional integration over metrics in Eq. (158) can be performed in a saddlepoint approximation,

$$
Z(\beta, Q, M)=e^{-W_{\text {tot }}[\beta, g(\beta)]},
$$

where metric $g_{\mu \nu}(\beta)$ is a solution to the saddle-point equation

$$
\frac{\delta W_{\mathrm{tot}}[\beta, g]}{\delta g}=0, W_{\mathrm{tot}}=W_{\mathrm{gr}}[\beta, g]+W[\beta, g]
$$

\footnotetext{
8 As I have recently learned (private communication from Myers), Jacobson and Myers (unpublished) had similar
} ideas back in the $1990 \mathrm{~s}$.

Living Reviews in Relativity

http: //www . livingreviews .org//rr-2011-8 
with the inverse temperature $\beta$ kept fixed. The solution of this equation is a regular (without conical singularities) metric $g_{\mu \nu}(\beta)$. This is the on-shell metric, which incorporates the quantum corrections due to the vacuum polarization by the matter fields. It can also be called an equilibrium configuration, which corresponds to the fixed temperature $\beta^{-1}$. In the saddle point approximation there is a constraint relating the charges at infinity $M$ and $Q$ and the inverse temperature $\beta$ : $\beta=\beta(M, Q)$.

The thermodynamic entropy is defined by the total response of the free energy $F=-\beta^{-1} \ln Z(\beta)$ to a small change in temperature,

$$
S_{\mathrm{TD}}=\beta^{2} d_{\beta} F=\left(\beta d_{\beta}-1\right) W_{\mathrm{tot}}\left(\beta, g_{\beta}\right)
$$

and involves, in particular, the derivative of the equilibrium configuration $g_{\mu \nu}(\beta)$ with respect to $\beta$

$$
d_{\beta} W_{\text {tot }}=\partial_{\beta} W_{\text {tot }}[\beta, g]+\frac{\delta W_{\text {tot }}[\beta, g]}{\delta g_{\mu \nu}} \frac{\delta g_{\mu \nu}}{\delta \beta}
$$

For an equilibrium configuration, satisfying Eq. (161), the second term in Eq. (163) vanishes and thus the total derivative with respect to $\beta$ coincides with a partial derivative.

Thus, in order to compute the thermodynamic entropy, one may proceed in two steps. First, for a generic metric, which satisfies the conditions i), ii) and iii) compute the off-shell entropy using the replica method, i.e., by introducing a small conical singularity at the horizon. This computation is done by taking a partial derivative with respect to $\beta$. Second, consider this off-shell entropy for an equilibrium configuration, which solves Eq. (161). Since for the classical gravitational action (112) one finds $\left(\beta \partial_{\beta}-1\right) W_{\mathrm{gr}}[\beta, g]=S\left(G_{B}, c_{i B}\right)$ (116) and for the quantum effective action one obtains the entanglement entropy $\left(\beta \partial_{\beta}-1\right) W[\beta, g]=S_{\text {ent }}$, the relation between the entanglement entropy and thermodynamic entropy is given by

$$
S_{\mathrm{TD}}=S\left(G_{B}, c_{i B}\right)+S_{\mathrm{ent}} .
$$

Therefore, the entanglement entropy constitutes only a (quantum) part of the thermodynamic entropy of the black hole. The thermodynamic entropy is defined for equilibrium configurations satisfying the quantum corrected Einstein equations (161). Thus, these configurations are not classical solutions to the Einstein equations but incorporate the quantum (one-loop) corrections. These configurations are regular metrics without conical singularities. The UV divergences in the free energy for these configurations are renormalized in a standard way and thus for the thermodynamic entropy the renormalization statement discussed above holds automatically ${ }^{9}$.

In flat spacetime the quantum (one-loop) thermodynamic and statistical entropies coincide as was shown by Allen [2] due to the fact that the corresponding partition functions differ by terms proportional to $\beta$. In the presence of black holes the exact relation between the two entropies has been a subject of some debate (see, for example, [86, 199]). However, the analysis made in [104] shows that in the presence of black hole the Euclidean and statistical free energies coincide, provided an appropriate method of regularization is used to regularize both quantities.

\section{2 't Hooft's brick-wall model}

In 1985 't Hooft [214] proposed a model, which was one of the first successful demonstrations that an entropy that scales as an area can be associated, in a rather natural way, to a black-hole horizon. The idea of 't Hooft's calculation was to consider a thermal gas of Hawking particles

\footnotetext{
9 This is true for minimally-coupled matter fields. In the presence of non-minimal couplings there appear extra terms in the thermodynamic entropy, which are absent in the entanglement entropy, as we discussed earlier in Section 3.16 .
} 
propagating just outside the black-hole horizon. The entropy in the canonical description of the system is calculated by means of the Wentzel-Kramers-Brillouin (WKB) approximation. Provided the temperature of the gas is equal to the Hawking temperature, the result of this calculation is unambiguous. However, there is an important subtlety: the density of states of a Hawking particle becomes infinite as one gets closer to the horizon. The reason for this is simple. Close to the horizon all particles effectively propagate in the optical metric. The later is conformally related to the black-hole metric

$$
d s_{\mathrm{BH}}^{2}=-g(r) d t^{2}+g^{-1}(r) d r^{2}+r^{2} d \omega_{d-2}
$$

as follows

$$
d s_{\mathrm{opt}}^{2}=g^{-1}(r) d s_{\mathrm{BH}}^{2}=-d t^{2}+g^{-2}(r) d r^{2}+r^{2} g^{-1}(r) d \omega_{d-2}^{2},
$$

where $d \omega_{d-2}^{2}$ is the metric of the $(d-2)$-unit sphere. In the optical metric, the near-horizon region, where the metric function in Eq. (165) can be approximated as

$$
g(r)=\frac{4 \pi}{\beta_{H}}\left(r-r_{+}\right)+O\left(r-r_{+}\right)^{2},
$$

occupies an infinite volume. Clearly, the infinite volume contains an infinite number of states. In order to regularize this infinity 't Hooft introduced a brick wall, an imaginary boundary at some small distance $\epsilon$ from the actual horizon. Then, the regularized optical volume is divergent when $\epsilon$ is taken to zero

$$
V_{\mathrm{opt}}=\Omega_{d-2} \int_{r_{\epsilon}} d r r^{d-2} g^{-d / 2} \sim A(\Sigma) \frac{\beta_{H}^{d-1}}{\epsilon^{d-2}}
$$

where $A(\Sigma)=r_{+}^{d-2} \Omega_{d-2}$ is the area of the horizon and $\epsilon \sim \sqrt{\beta_{H}\left(r_{\epsilon}-r_{+}\right)}$is the invariant distance between the brick wall $\left(r=r_{\epsilon}\right)$ and the actual horizon $\left(r=r_{+}\right)$. The entropy of a gas of massless particles at temperature $T=\beta_{H}^{-1}$ confined in volume $V_{\mathrm{opt}}$ in $d$ spacetime dimensions

$$
S_{B W} \sim V_{\mathrm{opt}} \beta_{H}^{1-d} \sim \frac{A(\Sigma)}{\epsilon^{d-2}}
$$

in the optical metric, is proportional to the horizon area. We should note that the universal behavior of the regularized optical volume (167) in the limit of small $\epsilon$ and its proportionality to the horizon area in this limit was important in establishing the result (168).

\subsubsection{WKB approximation, Pauli-Villars fields}

In the original calculation by 't Hooft one considers a minimally-coupled scalar field, which satisfies the Klein-Gordon equation

$$
\left(\nabla^{2}-m^{2}\right) \varphi=0
$$

on the background of a black-hole metric (165) and imposes a brick-wall boundary condition

$$
\varphi(x)=0 \text { at } r=r_{\epsilon} .
$$

Consider, for simplicity, the four-dimensional case. Expanding the scalar field in spherical coordinates $\varphi=e^{-i \omega t} Y_{l, m}(\theta, \phi) f(r)$ one finds that Eq. (169) becomes

$$
\omega^{2} g^{-1}(r) f(r)+r^{-2} \partial_{r}\left(r^{2} g(r) \partial_{r} f(r)\right)-\left(\frac{l(l+1)}{r^{2}}+m^{2}\right) f(r)=0 .
$$


One uses the WKB approximation in order to find a solution to this equation. In this approximation one represents $f(r)=\rho(r) e^{i S(r)}$, where $\rho(r)$ is a slowly-varying function of $r$ while $S(r)$ is a rapidlyvarying phase. One neglects derivatives of $\rho(r)$ and the second derivative of $S(r)$, one obtains the radial function in the form

$$
f(r)=\rho(r) e^{ \pm i \int^{r} \frac{d r}{g(r)} k(r, l, E)}, \quad k(r, l, E)=\sqrt{E^{2}-\left(m^{2}+\frac{l(l+1)}{r^{2}}\right) g(r)},
$$

valid in the region where $k^{2}(r) \geq 0$. The latter condition defines a maximal radius $r_{\omega, l}$, which is a solution to the equation $k^{2}\left(r_{\omega, l}\right)=0$. For a fixed value of the energy $E$, by increasing the mass $m$ of the particle or the angular momentum $l$, the radius $r_{\omega, l}$ approaches $r_{+}$so that the characteristic region where the solution (172) is valid is in fact the near horizon region. One imposes an extra Dirichlet condition $\varphi=0$ at $r=r_{\omega, l}$ so that the one-particle spectrum becomes discrete

$$
2 \int_{r_{\epsilon}}^{r_{\omega}, l} \frac{d r}{g(r)} k(r, \omega, l)=2 \pi n,
$$

where $n$ is an integer. This relation is used to count the number of one-particle states that correspond to fixed values of energy $\omega$ and angular momentum $l$,

$$
n(\omega, l)=\frac{1}{\pi} \int_{r_{\epsilon}}^{r_{\omega, l}} \frac{d r}{g(r)} k(r, \omega, l)
$$

Calculating the total number of states, which have the same energy $E$, one has to sum over $l$. This sum can be approximated by an integral

$$
n(\omega)=\int d l(2 l+1) n(\omega, l)=\frac{2}{3} \int_{r_{\epsilon}}^{r_{\omega}} \frac{r^{2}}{g^{2}(r)} k^{3}(r, \omega), \quad k(r, \omega)=\sqrt{\omega^{2}-m^{2} g(r)},
$$

where $r_{\omega}$ is determined by the condition that $k(r, \omega)=0$.

In the near horizon region one approximates the metric function $g(r)$ in Eq. (166) by the first two terms in the expansion in powers of $\left(r-r_{+}\right)$,

$$
g(r)=\frac{4 \pi}{\beta_{H}}\left(r-r_{+}\right)+C\left(r-r_{+}\right)^{2},
$$

where $\beta_{H}$ is the inverse Hawking temperature and $r_{+}$is the horizon radius. Constant $C$ is related to the curvature of spacetime near the horizon. The radial position of the brick wall is $r_{\epsilon}=r_{+}+\frac{\pi \epsilon^{2}}{\beta_{H}}$, where $\epsilon$ is the geodesic distance between the brick wall and the horizon. Focusing only on the brick-wall divergent terms, one obtains for the number of states (175)

$$
n(\omega)=\frac{r_{+}^{2} \beta_{H}^{3} \omega^{3}}{24 \pi^{4} \epsilon^{2}}+\left(\frac{r_{+}^{2} \beta_{H}^{2} \omega^{3}}{24 \pi^{4}}\left(\beta_{H} C-\frac{4 \pi}{r_{+}}\right)+\frac{r_{+}^{2} \beta_{H} m^{2} \omega}{2 \pi^{2}}\right) \ln \epsilon .
$$

In a thermal ensemble of scalar particles at fixed temperature $T=\beta^{-1}$, each state in the one-particle spectrum can be occupied by any integer number of quanta. One gets for the free energy

$$
\beta F=\int_{0}^{\infty} d \omega \frac{d n(\omega)}{d \omega} \ln \left(1-e^{-\beta \omega}\right)
$$

or, integrating by parts,

$$
\beta F=-\beta \int_{0}^{\infty} \frac{n(\omega)}{e^{\beta \omega}-1} d \omega
$$


Substituting Eq. (177) here and using the integrals

$$
\int_{0}^{\infty} \frac{d \omega \omega}{e^{\beta \omega}-1}=\frac{\pi^{2}}{6 \beta^{2}}, \quad \int_{0}^{\infty} \frac{d \omega \omega^{3}}{e^{\beta \omega}-1}=\frac{\pi^{4}}{15 \beta^{4}},
$$

one calculates the divergent terms in the free energy

$$
F=-\frac{r_{+}^{2}}{360 \epsilon^{2}} \frac{\beta_{H}^{3}}{\beta^{4}}-\left(\frac{r_{+}^{2}}{360}\left(\beta_{H} C-\frac{4 \pi}{r_{+}}\right) \frac{\beta_{H}^{2}}{\beta^{4}}+\frac{r_{+}^{2} m^{2}}{12} \frac{\beta_{H}}{\beta^{2}}\right) \ln \epsilon
$$

and, using equation $S=\left.\beta^{2} \partial_{\beta} F\right|_{\beta=\beta_{H}}$, the entropy

$$
S=\frac{r_{+}^{2}}{90 \epsilon^{2}}+\left(\frac{r_{+}^{2}}{90 \beta_{H}}\left(\beta_{H} C-\frac{4 \pi}{r_{+}}\right)+\frac{1}{6} r_{+}^{2} m^{2}\right) \ln \epsilon .
$$

Due to the relations

$$
C r_{+}^{2}-\frac{4 \pi r_{+}}{\beta_{H}}=\frac{1}{8 \pi} \int_{\Sigma}\left(R_{i i}-2 R_{i j i j}\right) \text { and } 4 \pi r_{+}^{2}=\int_{\Sigma} 1
$$

this expression for the entropy can be rewritten in a completely geometric form

$$
S=\frac{A(\Sigma)}{360 \pi \epsilon^{2}}+\frac{1}{720 \pi} \int_{\Sigma}\left(R_{i i}-2 R_{i j i j}+30 m^{2}\right) \ln \epsilon .
$$

The leading term proportional to the area was first calculated in the seminal paper of 't Hooft [214]. The area law in the brick-wall model was also studied in [173, 213, 9, 10, 178].

It is an important observation made by Demers, Lafrance and Myers in [62] that the brick-wall divergences are in fact the UV divergences. This can be seen in the Pauli-Villars regularization as was first done in [62]. Applying the Pauli-Villars regularization scheme for the four-dimensional scalar field theory studied here, one introduces five regulator fields $\left\{\varphi_{i}, i=1, \ldots, 5\right\}$ of different statistics and masses $\left\{m_{i}, i=1, \ldots, 5\right\}$ dependent on the UV cut-off $\mu$ [62]. Together with the original scalar $\varphi_{0}=\varphi\left(m_{0}=m\right)$ these fields satisfy two constraints

$$
\sum_{i=0}^{5} \Delta_{i}=0 m \text { and } \sum_{i=0}^{5} \Delta_{i} m_{i}^{2}=0
$$

where $\Delta_{i}=+1$ for the commuting fields, and $\Delta_{i}=-1$ for the anticommuting fields. Not deriving the exact expressions for $m_{i}$, we just quote here the following asymptotic behavior

$$
\begin{aligned}
& \sum_{i=0}^{5} \Delta_{i} m_{i}^{2} \ln m_{i}^{2}=\mu^{2} b_{1}+m^{2} \ln \frac{m^{2}}{\mu^{2}}+m^{2} b_{2}, \\
& \sum_{i=0}^{5} \Delta_{i} \ln m_{i}^{2}=\ln \frac{m^{2}}{\mu^{2}},
\end{aligned}
$$

(where $b_{1}$ and $b_{2}$ are some constants), valid in the limit $\mu \rightarrow \infty$. The total free energy is the sum of all contributions, from the original scalar field and the regulators

$$
\beta F=\beta \sum_{i=0}^{5} \Delta_{i} F^{i}
$$

It is clear that, due to the constraints (185), all brick-wall divergences (with respect to the parameter $\epsilon$ ) in the free energy (187) and in the entropy cancel. On the other hand, both the free 
energy and the entropy become divergent if the Pauli-Villars regulator $\mu$ is taken to infinity, thus confirming their identification as UV divergences. For the free energy one finds

$$
F=-\frac{1}{24} \frac{\beta_{H}}{\beta^{2}} r_{+}^{2} \sum_{i=0}^{5} \Delta_{i} M_{i}^{2} \ln M_{i}^{2}-\frac{1}{1440} \frac{\beta_{H}^{3}}{\beta^{4}} r_{+}^{2} C \sum_{i=0}^{5} \Delta_{i} \ln M_{i}^{2} .
$$

and for the entropy one has

$$
S=\frac{1}{48 \pi} A_{\Sigma} \sum_{i=0}^{5} \Delta_{i} m_{i}^{2} \ln m_{i}^{2}+\frac{1}{1440 \pi} \int_{\Sigma}\left(R_{i i}-2 R_{i j i j}\right) \sum_{i=0}^{5} \Delta_{i} \ln m_{i}^{2} .
$$

Several remarks are in order.

1) Comparing the entropy calculated in the brick-wall model, (184) or (189), with the entanglement entropy (82) we see that the structure of the UV divergent terms in two entropies is similar. The logarithmic terms in Eqs. (82) and (189) (or (184)) are identical if the black-hole metric has vanishing Ricci scalar, $R=0$. This is the case, for example, for the Reissner-Nordström black hole considered in [62]. However, the logarithmic terms in the two calculations are different if the Ricci scalar is non-zero. This discrepancy appears to arise due to certain limitations of the WKB approximation. In the exact solutions, known explicitly, for example, for a scalar field in a constant curvature spacetime, the mass $m$ always appears in combination $m^{2}-\frac{1}{6} R$. However, this is not seen in the WKB approximation (172). In fact, if one makes this substitution everywhere in the above brick-wall calculation, the Ricci scalar would appear in the brick-wall entropy in a manner, which agrees with the entanglement calculation (82). Moreover, an alternative calculation [104] of the density of states, which does not make use of the WKB approximation, results in an expression for the entropy, which agrees with Eq. (82).

2) The similarity between the two entropies suggests that the UV divergences in the brick-wall entropy (189) can be renormalized by the renormalization of the couplings in the gravitational action in the same way as for the entanglement entropy. That this indeed works was demonstrated in [62].

3) For a non-minimally-coupled field we have the same problem as in the case of the entanglement entropy. For metrics with $R=0$ the non-minimal coupling does not show up in the scalar field equation and does not change the density of states, the free energy and entropy. On the other hand, the non-minimal coupling affects the renormalization of Newton's constant, even if the background metric is Ricci flat. An attempt was made in [200] to modify the Dirichlet boundary condition at the brick wall and replace it by a more sophisticated condition, which would depend on the value of the non-minimal coupling $\xi$, so that the resulting entropy would have the UV divergences consistent with the renormalization of Newton's constant. However, this attempt cannot be considered as successful since it does not reproduce the expected behavior of the entropy for large positive values of $\xi$.

The calculation of the brick-wall entropy for a rotating black hole is more complicated due to the presence of the super- radiance modes in the spectrum. This issue is considered in [45, 92, 155, 41, 149, 224, 132, 148, 153].

\subsubsection{Euclidean path integral approach in terms of optical metric}

Field equation in optical metric. Consider a slightly more general equation than Eq. (169), by including a non-minimal coupling,

$$
\left(-\nabla^{2}+\xi R+m^{2}\right) \phi=0
$$

in the background of the black-hole metric $g_{\mu \nu}$, which takes the form (165). The optical metric is conformally related to the black-hole metric, $\bar{g}_{\mu \nu}=e^{2 \sigma} g_{\mu \nu}$, where $e^{2 \sigma}=1 /\left|g_{t t}\right|$ (in the metric (165) 
we have that $\left.g_{t t}=g(r)\right)$. Eq. (190) can be rewritten entirely in terms of the optical metric $\bar{g}_{\mu \nu}(166)$ as follows

$$
\begin{aligned}
& \left(\partial_{t}^{2}+\hat{H}^{2}\right) \varphi_{\mathrm{opt}}=0, \hat{H}^{2}=-\Delta_{\mathrm{opt}}+\mathcal{V}, \\
& \mathcal{V}=e^{-2 \sigma}\left(\left(\xi R+m^{2}\right)-\frac{(d-2)}{2} \nabla^{2} \sigma-\frac{(d-2)^{2}}{4}(\nabla \sigma)^{2}\right)
\end{aligned}
$$

where $\varphi_{\text {opt }}=e^{\frac{(d-2)}{4} \sigma} \varphi, \Delta_{\text {opt }}$ is the Laplace operator for spatial part $\gamma_{i j}^{o p t}=\bar{g}_{i j}$ of the optical metric, while the scalar curvature $R$ and the covariant derivative $\nabla$ are defined with respect to the original metric $g_{\mu \nu}=e^{-2 \sigma} \bar{g}_{\mu \nu}$. We notice that, since $e^{-2 \sigma}=g(r)$, the effective potential $\mathcal{V}$ in Eq. (191) vanishes at the horizon. This is a general feature of wave equations in the black-hole background: the fields become, effectively, massless in the near horizon region. The frequency $\omega$, which appears in Eq. (171) in the brick-wall calculation, is thus an eigenvalue of the operator $\hat{H}^{2}$,

$$
\hat{H}^{2} \varphi_{\omega}=\omega^{2} \varphi_{\omega}
$$

The canonical free energy and Euclidean path integral. The canonical free energy (178)

$$
F=\beta^{-1} \sum_{\omega} n(\omega) \ln \left(1-e^{-\beta \omega}\right),
$$

where $\omega$ are eigenvalues of the spatial operator $\hat{H}^{2}$ and $n(\omega)$ is the degeneracy of the energy level $\omega$, can be represented in terms of the Euclidean path integral for a field theory with wave operator $\left(\partial_{\tau}^{2}+\hat{H}^{2}\right)$, provided that the Euclidean time $\tau$ is a circle with period $\beta$. (This property was first clearly formulated by Allen [2].) It order to see this in a rather elementary way, we first notice that

$$
\ln \left(1-e^{-\beta \omega}\right)=-\frac{\beta \omega}{2}+\ln \beta \omega+\sum_{k=1}^{\infty} \ln \left(1+\frac{\omega^{2} \beta^{2}}{4 \pi^{2} k^{2}}\right)
$$

The sum in this expression can be rewritten as a difference of two sums

$$
\sum_{k=1}^{\infty} \ln \left(1+\frac{\omega^{2} \beta^{2}}{4 \pi^{2} k^{2}}\right)=\sum_{k=-\infty}^{\infty} \frac{1}{2} \ln \left(\omega^{2}+\frac{4 \pi^{2} k^{2}}{\beta^{2}}\right)-\sum_{k=1}^{\infty} \ln \left(\frac{4 \pi^{2} k^{2}}{\beta^{2}}\right)-\ln \omega
$$

Each of these sums should be understood in terms of the zeta-function regularization. In particular, using the properties of the Riemann $\zeta$-function, we find

$$
\sum_{k=1}^{\infty} \ln \left(\frac{4 \pi^{2} k^{2}}{\beta^{2}}\right)=\lim _{z \rightarrow 0} \frac{d}{d z} \sum_{k=1}^{\infty} \ln \left(\frac{4 \pi^{2} k^{2}}{\beta^{2}}\right)^{-z}=-\ln \beta
$$

Collecting together Eqs. (194), (195) and (196) one obtains that

$$
F=-\frac{1}{2} \sum_{\omega} n(\omega) \omega+\frac{1}{2 \beta} \sum_{\omega} n(\omega) \sum_{k=-\infty}^{\infty} \ln \left(\omega^{2}+\frac{4 \pi^{2} k^{2}}{\beta^{2}}\right)
$$

The second term in Eq. (197) can be expressed in terms of the Euclidean path integral. This can be seen as follows. In the Euclidean formulation one first makes a Wick rotation of time $t \rightarrow-i \tau$. The effective action $W_{\text {opt }}$ then is defined by means of the Euclidean path integral

$$
e^{-W_{\mathrm{opt}}}=\int \mathcal{D} \varphi_{\mathrm{opt}} e^{-\int \varphi_{\mathrm{opt}}\left(-\partial_{\tau}^{2}+\hat{H}^{2}\right) \varphi_{\mathrm{opt}}}
$$


At finite temperature one closes the Euclidean time by identifying $\tau$ and $\tau+\beta$. Then, the eigenvalues of operator $\partial_{\tau}$ then are $i \frac{2 \pi}{\beta} k$, where $k=0, \pm 1, \pm 2, \ldots$ The effective action can be expressed in terms of the logarithm of the determinant

$$
W_{\mathrm{opt}}=-\frac{1}{2} \ln \operatorname{det}\left(-\partial_{\tau}^{2}+\hat{H}^{2}\right)=-\frac{1}{2} \sum_{\omega} n(\omega) \sum_{k=-\infty}^{+\infty} \ln \left(\omega^{2}+\frac{4 \pi^{2} k^{2}}{\beta^{2}}\right) .
$$

Comparing this with Eq. (197) and defining the vacuum energy as

$$
E_{0}=\frac{1}{2} \sum_{\omega} n(\omega) \omega
$$

one arrives at an expression for the free energy (197)

$$
F=\beta^{-1} W_{\text {opt }}-E_{0} \text {. }
$$

Evaluation of the effective action in the optical metric. The effective action $W_{\text {opt }}$ (198) can be calculated using the heat kernel method. One notes that the heat kernel of the operator $-\partial_{\tau}^{2}+\hat{H}^{2}$ takes the form of a product of two heat kernels, for commuting operators $\partial_{\tau}^{2}$ and $\hat{H}^{2}$. The heat kernel for operator $\partial_{\tau}^{2}$ is computed explicitly, provided the periodicity condition, $\tau \rightarrow \tau+\beta$, is imposed. One finds for the trace

$$
\operatorname{Tr} e^{s \partial_{\tau}^{2}}=\frac{\beta}{\sqrt{4 \pi s}} \sum_{k=-\infty}^{\infty} e^{-\frac{k^{2} \beta^{2}}{4 s}}
$$

So that the effective action takes the form

$$
W_{\mathrm{opt}}=-\frac{1}{2} \int_{\epsilon^{2}}^{\infty} \frac{d s}{s} \frac{\beta}{\sqrt{4 \pi s}} \sum_{k=-\infty}^{\infty} e^{-\frac{k^{2} \beta^{2}}{4 s}} \operatorname{Tr} K_{\hat{H}^{2}}
$$

The operator $\hat{H}^{2}=-\Delta_{\text {opt }}+\mathcal{V}(191)$ is defined for the $(d-1)$-dimensional metric $\gamma_{i j}^{o p t}$, the spatial part of the optical metric (166). The trace of the heat kernel of operator $\hat{H}^{2}=-\Delta_{\text {opt }}+\mathcal{V}(191)$ can be represented as a series expansion in powers of $s$,

$$
\operatorname{Tr} K_{\hat{H}^{2}}=\frac{1}{(4 \pi s)^{\frac{d-1}{2}}}\left(\int \sqrt{\gamma_{\mathrm{opt}}}+s \int \sqrt{\gamma_{\mathrm{opt}}}\left(\frac{1}{6} R_{\mathrm{opt}}-\mathcal{V}\right)+O\left(s^{2}\right)\right),
$$

where the integration is taken over the spatial part of the optical metric (166) and $R_{\text {opt }}$ is the Ricci scalar of $(d-1)$-metric $\gamma_{i j}^{\mathrm{opt}}$.

For $n \neq 0$ the integration over the proper time $s$ in Eq. (203) is regularized for small $s$ due to the thermal exponential factor, so that the UV regulator $\epsilon$ can be removed. Interchanging the sum and the integral one obtains

$$
\sum_{n=1}^{\infty} \int_{0}^{\infty} \frac{d s}{s} s^{m-\frac{d}{2}} e^{-\frac{n^{2} \beta^{2}}{4 s}}=\left(\frac{\beta}{2}\right)^{2 m-d} \zeta(d-2 m) \Gamma\left(\frac{d}{2}-m\right), \quad m=0,1,2, . .
$$

Only the term with $n=0$ in Eq. (203) contains the UV divergences. This term in the effective action is proportional to the inverse temperature $\beta$ and thus it does not make any contribution to the entropy. On the other hand, the $n=0$ term gives the free energy at zero temperature. Thus, only the zero temperature contribution to the free energy is UV divergent as was shown by Dowker 
and Kennedy [73]. In fact the usual way to renormalize the free energy is to subtract the $n=0$ term in Eq. (203) or, equivalently, to subtract the zero temperature free energy, $F^{R}=F(T)-F(T=0)$. With this regularization and using Eq. (205) one obtains a sort of high temperature expansion of the effective action. In $d$ dimensions one finds for the regularized action [74, 75]

$$
W_{\mathrm{opt}}^{R}=-\frac{\beta^{1-d}}{\pi^{d / 2}} \zeta(d) \Gamma\left(\frac{d}{2}\right) V_{\mathrm{opt}}-\frac{\beta^{3-d}}{4 \pi^{d / 2}} \zeta(d-2) \Gamma\left(\frac{d}{2}-1\right) \int \sqrt{\gamma_{\mathrm{opt}}}\left(\frac{1}{6} R_{\mathrm{opt}}-\mathcal{V}\right)+O\left(\beta^{4-\phi(206)}\right.
$$

where, for $d=4$, the term $O\left(\beta^{4-d}\right)$ also contains a logarithmic term $\ln \beta$. In four dimensions the first two terms in Eq. (206) are the only terms in the effective action, which are divergent when the integration in the optical metric is taken up to the horizon. The first term in Eq. (206) and the respective term in the free energy and entropy is the contribution of a thermal gas in $(d-1)$ spatial volume $V_{\text {opt }}$ at temperature $T=\beta^{-1}$ in flat spacetime. The other terms in Eq. (206) are curvature corrections to the flat spacetime result as discussed by Dowker and Schofield [74, 75].

Let us focus on the four-dimensional case. Defining the regularized free energy $F^{R}=\beta^{-1} W_{\mathrm{opt}}^{R}$ and entropy $S_{\text {opt }}=\beta^{2} \partial_{\beta} F^{R}$ one finds (provided one imposes the condition $\beta=\beta_{H}$, after taking the derivative with respect to $\beta$ )

$$
S_{d=4}^{\mathrm{opt}}=\frac{2 \pi^{2}}{45} T_{H}^{3} V_{\mathrm{opt}}+\frac{1}{12} T_{H} \int \sqrt{\gamma_{\mathrm{opt}}}\left(\frac{1}{6} R_{\mathrm{opt}}-\mathcal{V}\right)
$$

where we omit terms, which are finite when the volume integration is extended to the horizon. The important observation now is that

$$
\int \sqrt{\gamma_{\mathrm{opt}}}\left(\frac{1}{6} R_{\mathrm{opt}}-\mathcal{V}\right)=\int \sqrt{\gamma_{\mathrm{opt}}} e^{-2 \sigma}\left(\left(\frac{1}{6}-\xi\right) R-m^{2}\right),
$$

where $e^{-2 \sigma}=g(r)$ and $R$ is the scalar curvature of the original black-hole metric. We recall that the latter is conformally related to the optical metric, $g_{\mu \nu}^{\text {opt }}=e^{2 \sigma} g_{\mu \nu}$, so the relation between the scalar curvature in two spacetimes is

$$
R_{\mathrm{opt}}=e^{-2 \sigma}\left(R-6 \nabla^{2} \sigma-6(\nabla \sigma)^{2}\right) .
$$

Using this relation and the form of the potential term $\mathcal{V}$ one arrives at Eq. (208).

Introducing the cut-off $\epsilon$ as before, $r_{\epsilon}=r_{+}+\frac{\pi \epsilon^{2}}{\beta_{H}}$, one finds for the volume in the optical metric

$$
V_{\mathrm{opt}}=\frac{\beta_{H}^{3} A(\Sigma)}{16 \pi^{3} \epsilon^{2}}+\frac{\beta_{H}}{32 \pi^{3}} \int_{\Sigma}\left(R_{i i}-2 R_{i j i j}\right) \ln \epsilon+O(\epsilon)
$$

and

$$
\int \sqrt{\gamma_{\mathrm{opt}}}\left(\frac{1}{6} R_{\mathrm{opt}}-\mathcal{V}=\left(\left(\frac{1}{6}-\xi\right) R\left(r_{+}\right)-m^{2}\right) \frac{\beta_{H}}{2 \pi} A_{+} \ln \epsilon^{-1}+O(\epsilon),\right.
$$

where $A_{+}=4 \pi r_{+}^{2}$ is the horizon area and $R\left(r_{+}\right)$is the value of the scalar curvature at the horizon.

Putting everything together, one finds that the entropy in the optical metric is

$$
S_{d=4}^{\mathrm{opt}}=\frac{A_{+}}{360 \pi \epsilon^{2}}+\int_{\Sigma}\left(\frac{1}{720 \pi}\left(R_{i i}-2 R_{i j i j}\right)-\frac{1}{24 \pi}\left(\left(\frac{1}{6}-\xi\right) R-m^{2}\right)\right) \ln \epsilon .
$$

Comparing this result with the entanglement entropy (82) computed earlier, we find complete agreement. Notice that $\epsilon$ here is in fact the IR regulator, the brick-wall cut-off, which regularizes the integration in the radial direction in the optical metric. As in 't Hooft's original calculation, 
this divergence can be transformed into a UV divergence by introducing the Pauli-Villars regulator fields of characteristic mass $\mu$. The UV divergences of the entropy when $\mu$ is taken to infinity are then of the same type as in Eq. (212). This was analyzed in [104].

The Euclidean path integral approach in the optical metric was considered in $[15,58,57,9,10$, $177,136]$.

We remind the reader that the entanglement entropy (82) is obtained by using the Euclidean path integral in the original black-hole metric with a conical singularity at the horizon (for $\beta \neq \beta_{H}$ ). The black-hole metric and the optical metric are related by a conformal transformation. This transformation is singular at the horizon and in fact produces a topology change: there appears a new boundary at $r=r_{+}$in the optical metric, which was a tip of the cone in the original black-hole metric. Because of this singular behavior of the conformal transformation, the exact relation between the two Euclidean path integrals is more subtle than for a regular conformal transformation. That the UV divergences in the entropy calculated in these two approaches coincide suggests that the equivalence between the two approaches might extend to the UV finite terms. Although in arbitrary dimension this equivalence may be difficult to prove, the analysis in two dimensions [199] shows that the entanglement entropy and the brick-wall entropy are indeed equivalent. This is, of course, consistent with the formal proof outlined in Section 3.6. 


\section{Some Particular Cases}

In this section we shall consider some particular examples in which the entanglement entropy, including the UV finite terms, can be calculated explicitly.

\subsection{Entropy of a 2D black hole}

In two dimensions the conformal symmetry plays a special role. This has many manifestations. In particular, the conformal symmetry can be used in order to completely reproduce, for a conformal field theory (CFT), the UV finite part of the corresponding gravitational effective action. This is done by integration of the conformal anomaly. For regular two-dimensional spacetimes, the result is the well-known non-local Polyakov action. In the presence of a conical singularity the derivation is essentially the same, although one has to take into account the contribution of the singularity. Consider a two-dimensional CFT characterized by a central charge $c$. For a regular two-dimensional manifold, the Polyakov action can be written in the form

$$
W_{\mathrm{PL}}[M]=\frac{c}{48 \pi} \int_{M}\left(\frac{1}{2}(\nabla \psi)^{2}+\psi R\right)
$$

where the field equation for the field $\psi$ is $\nabla^{2} \psi=R$. On a manifold $M^{\alpha}$ with a conical singularity with angle deficit $\delta=2 \pi(1-\alpha)$ the Polyakov action is modified by the contribution from the singularity at the horizon $\Sigma$ (which is just a point in two dimensions) so that $[196,112]$

$$
W_{\mathrm{PL}}\left[M^{\alpha}\right]=W_{\mathrm{PL}}\left[M^{\alpha} / \Sigma\right]+\frac{c}{12}(1-\alpha) \psi_{h}+O(1-\alpha)^{2},
$$

where $\psi_{h}$ is the value of the field $\psi$ on the horizon. Applying the replica method to the Polyakov action (214) one obtains that the corresponding contribution to the entanglement entropy from the UV finite term in the effective action is

$$
S_{\text {fin }}=\frac{c}{12} \psi_{h} .
$$

This result agrees with a derivation of Myers [179] who used the Noether charge method of Wald [221] in order to calculate the entropy. The easiest way to compute the function $\psi$ is to use the conformal gauge $g_{\mu \nu}=e^{2 \sigma} \delta_{\mu \nu}$ in which $\psi=2 \sigma$. Together with the UV divergent part, the complete entanglement entropy in two dimensions is

$$
S=\frac{c}{6} \sigma_{h}+\frac{c}{6} \ln \frac{\Lambda}{\epsilon},
$$

where $\Lambda$ is an IR cut-off.

Let the black-hole geometry be described by a $2 \mathrm{D}$ metric

$$
d s_{\mathrm{bh}}^{2}=f(x) d \tau^{2}+\frac{1}{f(x)} d x^{2},
$$

where the metric function $f(x)$ has a simple zero at $x=x_{+}$. Assume that this black hole is placed inside a box of finite size $L$ so that $x_{+} \leq x \leq L$. In order to get a regular space, one closes the Euclidean time $\tau$ with period $\beta_{H}, \beta_{H}=\frac{4 \pi}{f^{\prime}\left(x_{+}\right)}$. It is easy to see that Eq. (217) is conformal to the flat disk of radius $z_{0}\left(\ln z=\frac{2 \pi}{\beta_{H}} \int_{L}^{x} \frac{d x}{f(x)}\right)$ :

$$
\begin{aligned}
d s_{\mathrm{bh}}^{2} & =e^{2 \sigma} z_{0}^{2}\left(d z^{2}+z^{2} d \tilde{\tau}^{2}\right), \\
\sigma & =\frac{1}{2} \ln f(x)-\frac{2 \pi}{\beta_{H}} \int_{L}^{x} \frac{d x}{f(x)}+\ln \frac{\beta_{H}}{2 \pi z_{0}},
\end{aligned}
$$


where $\tilde{\tau}=\frac{2 \pi \tau}{\beta_{H}}(0 \leq \tilde{\tau} \leq 2 \pi), 0 \leq z \leq 1$. So that the entanglement entropy of the $2 \mathrm{D}$ black hole takes the form $[199,98]$

$$
S=\frac{c}{12} \int_{x_{+}}^{L} \frac{d x}{f(x)}\left(\frac{4 \pi}{\beta_{H}}-f^{\prime}\right)+\frac{c}{6} \ln \left(\frac{\beta_{H} f^{1 / 2}(L)}{\epsilon}\right),
$$

where we omit the irrelevant term that is a function of $\left(\Lambda, z_{0}\right)$ but not of the parameters of the black hole and have retained dependence on the UV regulator $\epsilon$.

As was shown in [199], the entanglement entropy (219) is identical to the entropy of the thermal atmosphere of quantum excitations outside the horizon in the "brick-wall" approach of 't Hooft [214].

The black hole resides inside a finite-sized box and $L$ is the coordinate of the boundary of the box. The coordinate invariant size of the subsystem complimentary to the black hole is $L_{\mathrm{inv}}=$ $\int_{x_{+}}^{L} d x / \sqrt{f(x)}$. Two limiting cases are of interest. In the first, the size of the system $L_{\text {inv }}$ is taken to infinity. Then, assuming that the black-hole spacetime is asymptotically flat, we obtain that the entanglement entropy (219) approaches the entropy of the thermal gas,

$$
S=\frac{c \pi}{3} L_{\mathrm{inv}} T_{H}
$$

This calculation illustrates an important feature of the entanglement entropy of a black hole placed in a box of volume $V$. Namely, the entanglement entropy contains a contribution of the thermal gas that, in the limit of large volume in dimension $d$, takes the form (7). This is consistent with the thermal nature of the reduced density matrix obtained from the Hartle-Hawking state by tracing over modes inside the horizon.

The other interesting case is when $L_{\mathrm{inv}}$ is small. In this case, we find the universal behavior

$$
S=\frac{c}{6}\left(\ln \frac{L_{\mathrm{inv}}}{\epsilon}+\frac{R\left(x_{+}\right)}{24} L_{\mathrm{inv}}^{2}+O\left(L_{\mathrm{inv}}^{3}\right)\right) .
$$

The universality of this formula lies in the fact that it does not depend on any characteristics of the black hole (mass, temperature) other than the value of the curvature $R\left(x_{+}\right)$at the horizon.

Consider two particular examples.

2D de Sitter spacetime is characterized by the metric function $f(x)=1-\frac{x^{2}}{l^{2}}$ and the Hawking temperature $T_{H}=1 / 2 \pi l$. In this spacetime the size of the box is bounded from above, $L_{\mathrm{inv}} \leq \pi l$. The corresponding entanglement entropy

$$
S_{d S_{2}}=\frac{c}{6} \ln \left(\frac{1}{\pi T_{H} \epsilon} \tan \left(T_{H} \pi L_{\mathrm{inv}}\right)\right)
$$

is a periodic function of $L_{\mathrm{inv}}$.

The string inspired black hole $[222,169]$ is described by the metric function $f(x)=1-$ $e^{-\lambda x}$. It described an asymptotically-flat spacetime. The Hawking temperature is $T_{H}=\frac{\lambda}{4 \pi}$. The entanglement entropy in this case is

$$
S_{\mathrm{str}}=\frac{c}{6} \ln \left(\frac{1}{2 \pi T_{H} \epsilon} \sinh \left(2 \pi T_{H} L_{\mathrm{inv}}\right)\right) .
$$

The entropy in these two examples resembles the entanglement entropy in flat spacetime at zero temperature (11) and at a finite temperature (12) respectively. 


\subsection{Entropy of 3D Banados-Teitelboim-Zanelli (BTZ) black hole}

\subsubsection{BTZ black-hole geometry}

The black-hole solution in three-dimensional gravity with negative cosmological constant was first obtained in [6] (see also [5] for global analysis of the solution). We start with the black-hole metric written in a form that makes it similar to the four-dimensional Kerr metric. Since we are interested in its thermodynamic behavior, we write the metric in the Euclidean form:

$$
d s^{2}=f(r) d \tau^{2}+f^{-1}(r) d r^{2}+r^{2}(d \phi+N(r) d \tau)^{2},
$$

where the metric functions $f(r)$ and $N(r)$ read

$$
f(r)=\frac{r^{2}}{l^{2}}-\frac{j^{2}}{r^{2}}-m=\frac{\left(r^{2}-r_{+}^{2}\right)\left(r^{2}+\left|r_{-}\right|^{2}\right)}{l^{2} r^{2}}, \quad N(r)=-\frac{j}{r^{2}}
$$

and we use the notation

$$
r_{+}^{2}=\frac{m l^{2}}{2}\left(1+\sqrt{1+\left(\frac{2 j}{m l}\right)^{2}}\right), \quad\left|r_{-}\right|^{2}=\frac{m l^{2}}{2}\left(\sqrt{1+\left(\frac{2 j}{m l}\right)^{2}}-1\right)
$$

Obviously one has that $r_{+}\left|r_{-}\right|=j l$. The coordinate $\phi$ in Eq. (224) is assumed to be periodic with period $2 \pi$.

In order to transform the metric (224) to a Lorentzian signature we need to make the analytic transformation $\tau \rightarrow i t, j \rightarrow-i j$ so that

$$
\begin{gathered}
r_{+} \rightarrow r_{+}^{L}=\sqrt{\frac{m l^{2}}{2}}\left(1+\sqrt{1-\left(\frac{2 j}{m l}\right)^{2}}\right)^{1 / 2}, \\
\left|r_{-}\right| \rightarrow \imath r_{-}^{L}=\sqrt{\frac{m l^{2}}{2}}\left(1-\sqrt{1-\left(\frac{2 j}{m l}\right)^{2}}\right)^{1 / 2},
\end{gathered}
$$

where $r_{+}^{L}$ and $r_{-}^{L}$ are the values in the Lorentzian spacetime. These are the respective radii of the outer and inner horizons of the Lorentzian black hole in $(2+1)$ dimensions. Therefore, we must always apply the transformation (227) after carrying out all calculations in the Euclidean geometry in order to obtain the result for the Lorentzian black hole. The Lorentzian version of the metric (224) describes a black hole with mass $m$ and angular momentum $J=2 j$. The outer horizon is located at $r=r_{+}$; the respective inverse Hawking temperature is

$$
\beta_{H}=\frac{4 \pi}{f^{\prime}\left(r_{+}\right)}=\frac{2 \pi r_{+} l^{2}}{r_{+}^{2}+\left|r_{-}\right|^{2}} .
$$

In the $(\tau, r)$ sector of the metric (224) there is no conical singularity at the horizon if the Euclidean time $\tau$ is periodic with period $\beta_{H}$. The horizon $\Sigma$ is a one-dimensional space with metric $d s_{\Sigma}^{2}=$ $l^{2} d \psi^{2}$, where $\psi=\frac{r_{+}}{l} \phi-\frac{\left|r_{-}\right|}{l^{2}} \tau$ is a natural coordinate on the horizon.

The BTZ space is obtained from the three-dimensional maximally-symmetric hyperbolic space $H_{3}$ (sometimes called the global Euclidean anti-de Sitter space) by making certain identifications. In order to see this one may use the coordinate transformation

$$
\begin{aligned}
& \psi=\frac{r_{+}}{l} \phi-\frac{\left|r_{-}\right|}{l^{2}} \tau, \quad \theta=\frac{r_{+}}{l} \tau+\frac{\left|r_{-}\right|}{l^{2}} \phi \\
& \cosh ^{-1} \rho=\left(\frac{r_{+}^{2}+\left|r_{-}\right|^{2}}{r^{2}+\left|r_{-}\right|^{2}}\right)^{1 / 2} .
\end{aligned}
$$


In new coordinates $(\rho, \theta, \psi)$ the BTZ metric takes the form

$$
d s^{2}=l^{2}\left(d \rho^{2}+\cosh ^{2} \rho d \psi^{2}+\sinh ^{2} \rho d \theta^{2}\right),
$$

which is the metric on the hyperbolic space $H_{3}$. In this metric the BTZ geometry is defined by identifications

i) $\theta \rightarrow \theta+2 \pi$

ii) $\theta \rightarrow \theta+2 \pi \frac{\left|r_{-}\right|}{l}, \quad \psi \rightarrow \psi+2 \pi \frac{r_{+}}{l}$.

The outer horizon $r=r_{+}$in the coordinate system $(\rho, \theta, \psi)$ is located at $\rho=0$ and $\psi$ is the angular coordinate on the horizon. Notice that the geodesic distance $\sigma$ between two points with coordinates $(\rho, \psi, \theta)$ and $\left(\rho, \psi^{\prime}, \theta^{\prime}\right)$ is

$$
\sinh ^{2} \frac{\sigma}{2 l}=\cosh ^{2} \rho \sinh ^{2} \frac{\psi-\psi^{\prime}}{2}+\sinh ^{2} \rho \sin ^{2} \frac{\theta-\theta^{\prime}}{2} .
$$

\subsubsection{Heat kernel on regular BTZ geometry}

Consider a scalar field with the operator $\mathcal{D}=-\left(\nabla^{2}+\xi / l^{2}\right)$. The maximally-symmetric constantcurvature space is a nice example of a curved space in which the heat equation $\left(\partial_{s}+\mathcal{D}\right) K\left(x, x^{\prime}, s\right)=$ 0 has a simple, exact, solution. The heat kernel in this case is a function of the geodesic distance $\sigma$ between two points $x$ and $x^{\prime}$. On the global space $H_{3}$ one finds

$$
K_{H_{3}}(\sigma, s)=\frac{1}{(4 \pi s)^{3 / 2}} \frac{\sigma / l}{\sinh (\sigma / l)} e^{-\frac{\sigma^{2}}{4 s}-\mu \frac{s}{l^{2}}},
$$

where $\mu=1-\xi$. The regular BTZ geometry is defined by identifications $i$ and $i i$ defined above. As is seen from Eq. (231) the geodesic distance and the heat kernel (232), expressed in coordinates $(\rho, \psi, \theta)$, are automatically invariant under identification $i$. Thus, it remains to maintain identification $i i$. This is done by summing over images

$$
K_{\mathrm{BTZ}}\left(x, x^{\prime}, s\right)=\sum_{n=-\infty}^{+\infty} K_{H_{3}}\left(\rho, \rho^{\prime}, \psi-\psi^{\prime}+2 \pi \frac{r_{+}}{l} n, \theta-\theta^{\prime}+2 \pi \frac{\left|r_{-}\right|}{l} n\right) .
$$

Using the path integral representation of the heat kernel we would say that the $n=0$ term in Eq. (233) is due to the direct way of connecting points $x$ and $x^{\prime}$ in the path integral. On the other hand, the $n \neq 0$ terms are due to uncontractible winding paths that go $n$ times around the circle.

\subsubsection{Heat kernel on conical BTZ geometry}

The conical BTZ geometry, which is relevant to the entanglement entropy calculation, is obtained from global hyperbolic space $H_{3}$ by replacing identification $i$ as follows

$$
\left.i^{\prime}\right) \theta \rightarrow \theta+2 \pi \alpha
$$

and not changing identification $i i$. For $\alpha \neq 1$ this Euclidean space has a conical singularity at the horizon $(\rho=0)$. The heat kernel on the conical BTZ geometry is constructed via the heat kernel (233) on the regular BTZ space by means of the Sommerfeld formula (22)

$$
K_{\mathrm{BTZ}_{\alpha}}\left(x, x^{\prime}, s\right)=K_{\mathrm{BTZ}}\left(x, x^{\prime}, s\right)+\frac{1}{4 \pi \alpha} \int_{\Gamma} \cot \frac{w}{2 \alpha} K_{\mathrm{BTZ}}\left(\theta-\theta^{\prime}+w, s\right) d w,
$$

where $K_{\mathrm{BTZ}}$ is the heat kernel (233). The contour $\Gamma$ is defined in Eq. (22). 
For the trace of the heat kernel (234) one finds [171] after computing by residues the contour integral

$$
\begin{aligned}
\operatorname{Tr} K_{\mathrm{BTZ}}= & \left(\frac{V_{B T Z_{\alpha}}}{l^{3}}+\frac{A_{+}}{l}(2 \pi \alpha) c_{2}(\alpha) \bar{s}\right) \frac{e^{-\mu \bar{s}}}{(4 \pi \bar{s})^{3 / 2}} \\
& +2 \pi \frac{e^{-\mu \bar{s}}}{(4 \pi \bar{s})^{3 / 2}} \frac{A_{+}}{l} \bar{s} \sum_{n=1}^{\infty} \frac{\sinh \frac{\Delta \psi_{n}}{\alpha}}{\sinh \Delta \psi_{n}} \frac{e^{-\frac{\Delta \psi_{n}^{2}}{4 \bar{s}}}}{\left(\sinh ^{2} \frac{\Delta \psi_{n}}{2 \alpha}+\sinh ^{2} \frac{\gamma_{n}}{2 \alpha}\right)},
\end{aligned}
$$

where $\bar{s}=s / l^{2}, \gamma_{n}=A_{-} n / l$ and $\Delta \psi_{n}=A_{+} n / l\left(A_{+}=2 \pi r_{+}\right.$and $\left.A_{-}=2 \pi r_{-}\right)$. Notice that we have already made the analytical continuation to the values of $r_{+}$and $r_{-}$in the Lorentzian geometry.

\subsubsection{The entropy}

When the trace of the heat kernel on the conical geometry is known one may compute the entanglement entropy by using the replica trick. Then, the entropy is the sum of UV divergent and UV finite parts [171]

$$
S_{\mathrm{ent}}=S_{\mathrm{div}}+S_{\mathrm{fin}}
$$

where the UV divergent part is

$$
S_{\mathrm{div}}=\frac{A_{+}}{24 \sqrt{\pi}} \int_{\epsilon^{2}}^{\infty} \frac{d s}{s^{3 / 2}} e^{-\mu s / l^{2}}=\frac{A_{+}}{12 \sqrt{\pi}}\left(\epsilon^{-1}-\frac{\sqrt{\mu \pi}}{l}\right) .
$$

This divergence is renormalized by the standard renormalization of Newton's constant

$$
\frac{1}{16 \pi G_{\mathrm{ren}}}=\frac{1}{16 \pi G_{B}}+\frac{1}{12} \frac{1}{(4 \pi)^{3 / 2}} \int_{\epsilon^{2}}^{\infty} \frac{d s}{s^{3 / 2}} e^{-\mu s / l^{2}}
$$

in the three-dimensional gravitational action.

The UV finite part in the entropy is

$$
\begin{aligned}
S_{\mathrm{fin}} & =\sum_{n=1}^{\infty} s_{n}, \\
s_{n} & =\frac{1}{2 n} \frac{e^{-\sqrt{\mu} \bar{A}_{+} n}}{\left(\cosh \bar{A}_{+} n-\cosh \bar{A}_{-} n\right)}\left(1+\bar{A}_{+} n \operatorname{coth} \bar{A}_{+} n-\frac{\left(\bar{A}_{+} n \sinh \bar{A}_{+} n-\bar{A}_{-} n \sinh \bar{A}_{-} n\right)}{\left(\cosh \bar{A}_{+} n-\cosh \bar{A}_{-} n\right)}\right)(239
\end{aligned}
$$

where $\bar{A}_{ \pm}=2 \pi r_{ \pm} / l$.

After the renormalization of Newton's constant, the complete entropy of the BTZ black hole, $S_{\mathrm{BTZ}}=S_{\mathrm{BH}}+S_{\mathrm{ent}}$, is a rather complicated function of the area of inner and outer horizons. Approximating in Eq. (239) the infinite sum by an integral one finds [171]

$$
\begin{aligned}
S & =\frac{A_{+}}{4 G_{\mathrm{ren}}}+\int_{\bar{A}_{+}}^{\infty} s(x) d x, \\
s(x) & =\frac{1}{2 x} \frac{e^{-\sqrt{\mu} x}}{(\cosh x-\cosh k x)}\left(1+x \operatorname{coth} x-\frac{(x \sinh x-k x \sinh k x)}{(\cosh x-\cosh k x)}\right),
\end{aligned}
$$

where $k=A_{-} / A_{+}$. The second term on the right-hand side of Eq. (240) can be considered to be the one-loop quantum (UV-finite) correction to the classical entropy of a black hole. 
For large enough $\bar{A}_{+} \equiv \frac{A_{+}}{l}>>1$ the integral in Eq. (240) goes to zero exponentially and we have the classical Bekenstein-Hawking formula for entropy. On the other hand, for small $\bar{A}_{+}$, the integral in Eq. (240) behaves logarithmically so that one has [171]

$$
S_{\mathrm{BTZ}}=\frac{A_{+}}{4 G}+\frac{\sqrt{\mu}}{6} \frac{A_{+}}{l}-\frac{1}{6} \ln \frac{A_{+}}{l}+O\left(\left(\frac{A_{+}}{l}\right)^{2}\right)
$$

This logarithmic behavior for small values of $A_{+}$(provided the ratio $k=A_{-} / A_{+}$is fixed) is universal and independent of the constant $\xi($ or $\mu$ ) in the field operator and the area of the inner horizon $\left(A_{-}\right)$of the black hole. Hence, the rotation parameter $J$ enters Eq. (241) only via the area $A_{+}$of the outer horizon.

The other interesting feature of the entropy (240) is that it always develops a minimum, which is a solution to the equation

$$
\frac{l}{4 G_{\mathrm{ren}}}=s\left(\frac{A_{+}}{l}\right) .
$$

This black hole of minimal entropy may be interesting in the context of the final stage of the Hawking evaporation in three dimensions. As follows from the analysis of Mann and Solodukhin [171], the minimum of the entropy occurs for a black hole whose horizon area is of the Planck length, $A_{+} \sim l_{\mathrm{PL}}$ (in threee dimensions $\left.l_{\mathrm{PL}} \sim G_{\mathrm{ren}}\right)$.

\subsection{Entropy of d-dimensional extreme black holes}

The extremal black holes play a special role in gravitational theory. These black holes are characterized by vanishing Hawking temperature $T_{H}$, which means that in the metric (165) the near-horizon expansion in the metric function $g(r)$ starts with the quadratic term $\left(r-r_{+}\right)^{2}$. Topologically, the true extremal geometry is different from the non-extremal one. Near the horizon the non-extremal static geometry looks like a product of a two-dimensional disk (in the plane $(r, \tau)$ ) and a $(d-2)$ dimensional sphere. Then, the horizon is the center in the polar coordinate system on the disk. Contrary to this, an extremal geometry in the near-horizon limit is a product of a two-dimensional cylinder and a $(d-2)$-dimensional sphere. Thus, the horizon in the extremal case is just another boundary rather than a regular inner point, as in the non-extremal geometry. However, one may consider a certain limiting procedure in which one approaches the extremal case staying all the time in the class of non-extremal geometries. This limiting procedure is what we shall call the "extremal limit". A concrete procedure of this type was suggested by Zaslavsky [226]. One considers a sequence of non-extreme black holes in a cavity at $r=r_{B}$ and finds that there exists a set of data $\left(r_{+}, r_{B}, r_{-}\right)$such that the limit $\frac{r_{+}}{r_{-}} \rightarrow 1, \frac{r_{B}}{r_{+}} \rightarrow 1$ is well defined. Even if one may have started with a rather general non-extremal metric, the limiting geometry is characterized by very few parameters. In this sense, one may talk about "universality" of the extremal limit. In fact, in the most interesting (and tractable) case the limiting geometry is the product of two-dimensional hyperbolic space $\mathrm{H}_{2}$ with the $(d-2)$-dimensional sphere. Since the limiting geometry belongs to the non-extreme class, its classical entropy is proportional to the horizon area in accord with the Bekenstein-Hawking formula. Then, the entanglement entropy of the limiting geometry is a one-loop quantum correction to the classical result. The universality we have just mentioned suggests that this correction possesses a universal behavior in the extreme limit and, since the limiting geometry is rather simple, the limiting entropy can be found explicitly. The latter was indeed shown by Mann and Solodukhin in [172]. 


\subsubsection{Universal extremal limit}

Consider a static spherically-symmetric metric in the following form

$$
d s^{2}=g(r) d \tau^{2}+\frac{1}{g(r)} d r^{2}+r^{2} d \omega_{d-2}^{2},
$$

where $d \omega_{d-2}^{2}$ is the metric on the $(d-2)$-dimensional unit sphere, describing a non-extreme hole with an outer horizon located at $r=r_{+}$. However, the analysis can be made for a more general metric, in which $g_{\tau \tau} \neq g_{r r}^{-1}$, the limiting geometry is the simplest in the case we consider in Eq. (243). The function $g(r)$ in Eq. (243) can be expanded as follows

$$
g(r)=a\left(r-r_{+}\right)+b\left(r-r_{+}\right)^{2}+O\left(\left(r-r_{+}\right)^{2}\right) .
$$

It is convenient to consider the geodesic distance $l=\int g^{-1 / 2} d r$ as a radial coordinate. Retaining the first two terms in Eq. (244), we find, for $r>r_{+}$, that

$$
\begin{aligned}
& \left(r-r_{+}\right)=\frac{a}{b} \sinh ^{2}\left(\frac{l b^{1 / 2}}{2}\right), \\
& g\left(l b^{1 / 2}\right)=\left(\frac{a^{2}}{4 b}\right) \sinh ^{2}\left(l b^{1 / 2}\right) .
\end{aligned}
$$

In order to avoid the appearance of a conical singularity at $r=r_{+}$, the Euclidean time $\tau$ in Eq. (243) must be compactified with period $4 \pi / a$, which goes to infinity in the extreme limit $a \rightarrow 0$. However, rescaling $\tau \rightarrow \phi=\tau a / 2$ yields a new variable $\phi$ having period $2 \pi$. Then, taking into account Eq. (245), one finds for the metric (243)

$$
d s^{2}=\frac{1}{b}\left(\sin ^{2} x d \phi^{2}+d x^{2}\right)+\left(r_{+}+\frac{a}{b} \sinh ^{2} \frac{x}{2}\right)^{2} d \omega_{d-2}^{2},
$$

where we have introduced the variable $x=l b^{1 / 2}$. To obtain the extremal limit one just takes $a \rightarrow 0$. The limiting geometry

$$
d s^{2}=\frac{1}{b}\left(\sin ^{2} x d \phi^{2}+d x^{2}\right)+r_{+}^{2} d \omega_{d-2}^{2}
$$

is that of the direct product of a 2-dimensional space and a $(d-2)$-sphere and is characterized by a pair of dimensional parameters $b^{-1 / 2}$ and $r_{+}$. The parameter $r_{+}$sets the radius of the $(d-2)$-dimensional sphere, while the parameter $b^{-1 / 2}$ is the curvature radius for the $(x, \phi) 2$ space. Clearly, this two-dimensional space is the negative constant curvature space $H_{2}$. This is the universality we mentioned above: although the non-extreme geometry is in general described by an infinite number of parameters associated with the determining function $g(r)$, the geometry in the extreme limit depends only on two parameters $b$ and $r_{+}$. Note that the coordinate $r$ is inadequate for describing the extremal limit (247) since the coordinate transformation (245) is singular when $a \rightarrow 0$. The limiting metric (247) is characterized by a finite temperature, determined by the $2 \pi$ periodicity in angular coordinate $\phi$.

The limiting geometry (247) is that of a direct product $H_{2} \times S_{2}$ of 2 d hyperbolic space $H_{2}$ with radius $l=b^{-1 / 2}$ and a $2 \mathrm{D}$ sphere $S_{2}$ with radius $l_{1}=r_{+}$. It is worth noting that the limiting geometry (247) precisely merges near the horizon with the geometry of the original metric (243) in the sense that all the curvature tensors for both metrics coincide. This is in contrast with, say, the situation in which the Rindler metric is considered to approximate the geometry of a non-extreme black hole: the curvatures of both spaces do not merge in general.

For a special type of extremal black holes $l=l_{1}$, the limiting geometry is characterized by just one dimensionful parameter. This is the case for the Reissner-Nordström black hole in four dimensions. The limiting extreme geometry in this case is the well-known Bertotti-Robinson space characterized by just one parameter $r_{+}$. This space has remarkable properties in the context of supergravity theory that are not the subject of the present review.

Living Reviews in Relativity

http://www. livingreviews.org/lrr-2011-8 


\subsubsection{Entanglement entropy in the extremal limit}

Consider now a scalar field propagating on the background of the limiting geometry (247) and described by the operator

$$
\mathcal{D}=-\left(\nabla^{2}+X\right), \quad X=-\xi R_{(d)},
$$

where $R_{(d)}$ is the Ricci scalar. For the metric (247) characterized by two dimensionful parameters $l$ and $l_{1}$, one has that $R_{(d)}=-2 / l^{2}+(d-2)(d-3) / l_{1}^{2}$. For a $d$-dimensional conformally-coupled scalar field we have $\xi=\frac{d-2}{4(d-1)}$. In this case

$$
X_{\mathrm{conf}}=-\frac{(d-2)(d-4)}{4 l^{2}}+\frac{(d-2)}{2(d-1)}\left(1 / l^{2}-1 / l_{1}^{2}\right) .
$$

The calculation of the respective entanglement entropy goes along the same lines as before. First, one allows the coordinate $\phi$, which plays the role of the Euclidean time, to have period $2 \pi \alpha$. For $\alpha \neq 1$ the metric (247) then describes the space $E^{\alpha}=H_{2}^{\alpha} \times S_{d-2}$, where $H_{2}^{\alpha}$ is the hyperbolic space coinciding with $\mathrm{H}_{2}$ everywhere except the point $x=0$, where it has a conical singularity with an angular deficit $\delta=2 \pi(1-\alpha)$. The heat kernel of the Laplace operator $\nabla^{2}$ on $E^{\alpha}$ is given by the product

$$
K_{E^{\alpha}}\left(z, z^{\prime}, s\right)=K_{H_{2}^{\alpha}}\left(x, x^{\prime}, \phi, \phi^{\prime}, s\right) K_{S_{d-2}}\left(\theta, \theta^{\prime}, \varphi, \varphi^{\prime}, s\right)
$$

where $K_{H_{2}^{\alpha}}$ and $K_{S^{2}}$ are the heat kernels, of the Laplace operator on $H_{2}^{\alpha}$ and $S_{d-2}$ respectively. The effective action reads

$$
W_{\mathrm{eff}}\left[E^{\alpha}\right]=-\frac{1}{2} \int_{\epsilon^{2}}^{\infty} \frac{d s}{s} \operatorname{Tr} K_{H_{2}^{\alpha}} \operatorname{Tr} K_{S_{d-2}} e^{X s},
$$

where $\epsilon$ is a UV cut-off. On spaces with constant curvature the heat kernel function is known explicitly [34]. In particular, on a 2D space $H_{2}$ of negative constant curvature, the heat kernel has the following integral representation:

$$
K_{H_{2}}\left(z, z^{\prime}, s\right)=\frac{1}{l^{2}} \frac{\sqrt{2} e^{-\bar{s} / 4}}{(4 \pi \bar{s})^{3 / 2}} \int_{\sigma}^{\infty} \frac{d y y e^{-y^{2} / 4 \bar{s}}}{\sqrt{\cosh y-\cosh \sigma}},
$$

where $\bar{s}=s l^{-2}$. In Eq. (251) $\sigma$ is the geodesic distance between the points on $H_{2}$. Between two points $(x, \phi)$ and $(x, \phi+\Delta \phi)$ the geodesic distance is given by $\sinh ^{2} \frac{\sigma}{2}=\sinh ^{2} x \sin ^{2} \frac{\Delta \phi}{2}$. The heat kernel on the conical hyperbolic space $H_{2}^{\alpha}$ can be obtained from (251) by applying the Sommerfeld formula (22). Skipping the technical details, available in [172], let us just quote the result for the trace

$$
\begin{aligned}
& \operatorname{Tr} K_{H_{2}^{\alpha}}=\alpha \operatorname{Tr} K_{H_{2}}+(1-\alpha) \frac{e^{-\bar{s} / 4}}{(4 \pi \bar{s})^{1 / 2}} k_{H}(\bar{s})+O(1-\alpha)^{2}, \\
& k_{H}(\bar{s})=\int_{0}^{\infty} d y \frac{\cosh y}{\sinh ^{2} y}\left(1-\frac{2 y}{\sinh 2 y}\right) e^{-y^{2} / \bar{s}}
\end{aligned}
$$

where $\bar{s}=s / l^{2}$.

Let us denote $\Theta_{d-2}(s)=\operatorname{Tr} K_{S_{d-2}}(s)$ the trace of the heat kernel of the Laplace operator $-\nabla^{2}$ on a $(d-2)$-dimensional sphere of unit radius. The entanglement entropy in the extremal limit then takes the form $[172,206]$

$$
S_{\mathrm{ext}}=\frac{1}{4 \sqrt{\pi}} \int_{\epsilon^{2} / l^{2}}^{\infty} \frac{d s}{s^{3 / 2}} k_{H}(s) \Theta_{d-2}\left(s \frac{l^{2}}{l_{1}^{2}}\right) e^{-s / 4} e^{s X l^{2}} .
$$


The function $k_{H}(s)$ has the following small-s expansion

$$
k_{H}(s)=\sqrt{\pi s}\left(\frac{1}{3}-\frac{1}{20} s+\frac{17}{1120} s^{2}-\frac{29}{4480} s^{3}+\frac{1181}{337920} s^{4}-\frac{1393481}{615014400} s^{5}+\frac{763967}{447283200} s^{6}+.(254)\right.
$$

The trace of the heat kernel on a sphere is known in some implicit form. However, for our purposes a representation in a form of an expansion is more useful,

$$
\Theta_{d-2}(s)=\frac{\Omega_{d-2}}{(4 \pi s)^{(d-2) / 2}}\left(1+(d-2)(d-3) \sum_{n=1}^{\infty} a_{2 n} s^{n}\right)
$$

where $\Omega_{d-2}=\frac{2 \pi^{(d-1) / 2}}{\Gamma((d-1) / 2)}$ is the area of a unit radius sphere $S_{d-2}$. The first few coefficients in this expansion can be calculated using the results collected in [219],

$$
a_{2}=\frac{1}{6}, \quad a_{4}=\frac{\left(5 d^{2}-27 d+40\right)}{360}, \quad a_{6}=\frac{\left(35 d^{4}-392 d^{3}+1699 d^{2}-3322 d+2520\right)}{45360} .
$$

We shall consider some particular cases.

$\boldsymbol{d}=4$. The entanglement entropy in the extreme limit is

$$
S_{d=4}=\frac{l_{1}^{2}}{12 \epsilon}+s_{0} \ln \frac{\epsilon}{l}+s\left(\frac{l_{1}}{l}\right), \quad s_{0}=\frac{1}{18}(6 \xi-1)+\frac{1}{15} \frac{l_{1}^{2}}{l^{2}}(1-5 \xi),
$$

where $s\left(\frac{l_{1}}{l}\right)$ is the UV finite part of the entropy. For minimal coupling $(\xi=0)$ this result was obtained in [172]. The first term in Eq. (257) is proportional to the horizon area $A=4 \pi l_{1}^{2}$, while the second term is a logarithmic correction to the area law. For conformal coupling $\xi=1 / 6$, the logarithmic term is

$$
s_{0}^{\mathrm{conf}}=\frac{1}{90} \frac{l_{1}^{2}}{l^{2}}
$$

$\boldsymbol{d}=\mathbf{5}$. The entropy is

$$
S_{d=5}=\frac{\sqrt{\pi} l_{1}^{3}}{72 \epsilon^{3}}+\frac{\sqrt{\pi}}{120}\left(\left(2 l_{1}^{2}-5 l^{2}\right)+10 \xi\left(3 l^{2}-l_{1}^{2}\right)\right) \frac{l_{1}}{l^{2} \epsilon}+s\left(\frac{l_{1}}{l}\right) .
$$

To simplify the expressions in higher dimensions we consider only the case of the conformal coupling $\xi=\frac{d-2}{4(d-1)}$.

The entropy takes the form:

$d=6$.

$$
\begin{aligned}
S_{d=6} & =\frac{l_{1}^{4}}{144 \epsilon^{4}}+\frac{1}{180} \frac{l_{1}^{2}}{l^{2}} \frac{\left(4 l^{2}-5 l_{1}^{2}\right)}{\epsilon^{2}}+s_{0} \ln \frac{\epsilon}{l_{1}}+s\left(\frac{l}{l_{1}}\right) \\
s_{0} & =-\frac{1}{18900}\left(1068 \frac{l_{1}^{4}}{l^{4}}-1680 \frac{l_{1}^{2}}{l^{2}}+637\right) .
\end{aligned}
$$


$d=7$.

$$
\begin{aligned}
S_{d=7}= & \frac{\sqrt{\pi} l_{1}^{5}}{384 \epsilon^{5}}+\frac{7 \sqrt{\pi}}{34560} \frac{\left(25 l^{2}-32 l_{1}^{2}\right) l_{1}^{3}}{l^{2} \epsilon^{3}} \\
& +\frac{\sqrt{\pi}}{1935360} \frac{\left(70592 l_{1}^{4}-109760 l_{1}^{2} l^{2}+40635 l^{4}\right) l_{1}}{l^{5} \epsilon}+s\left(\frac{l_{1}}{l}\right) .
\end{aligned}
$$

$d=8$.

$$
\begin{aligned}
S_{d=8} & =\frac{s_{6}}{\epsilon^{6}}+\frac{s_{4}}{\epsilon^{4}}+\frac{s_{2}}{\epsilon^{2}}+s_{0} \ln \epsilon+s\left(\frac{l_{1}}{l}\right), \\
s_{6} & =\frac{l_{1}^{6}}{2160}, s_{4}=\frac{1}{75600} \frac{l_{1}^{4}\left(-209 l_{1}^{2}+160 l^{2}\right)}{l^{6}}, \\
s_{2} & =\frac{1}{352800} \frac{l_{1}^{2}\left(8753 l_{1}^{4}-13376 l^{2} l_{1}^{2}+4875 l^{4}\right)}{l^{6}}, \\
s_{0} & =\frac{1}{11113200} \frac{\left(1102263 l_{1}^{6}-2520864 l^{2} l_{1}^{4}+1833975 l^{4} l_{1}^{2}-413120 l^{6}\right)}{l^{6}} .
\end{aligned}
$$

Two examples of the extreme geometry are of particular interest.

Entanglement entropy of the round sphere in Minkowski spacetime. Consider a sphere of radius $R$ in flat Minkowski spacetime. One can choose a spherical coordinate system $\left(\tau, r, \theta^{i}\right)$ so that the surface $\Sigma$ is defined as $\tau=0$ and $r=R$, and variables $\theta^{i}, i=1, . ., d-2$ are the angular coordinates on $\Sigma$. The $d$-metric reads

$$
d s^{2}=d \tau^{2}+d r^{2}+r^{2} d \omega_{d-2}^{2}
$$

where $d \omega_{d-2}^{2}$ is a metric on $(d-2)$ sphere of unit radius. Metric (264) is conformal to the metric

$$
d s_{\mathrm{ext}}^{2}=\frac{R^{2}}{r^{2}}\left(d \tau^{2}+d r^{2}\right)+R^{2} d \omega_{d-2}^{2},
$$

which describes the product of two-dimensional hyperbolic space $H_{2}$ with coordinates $(\tau, r)$ and the sphere $S_{d-2}$. Note that both spaces, $H_{2}$ and $S_{d-2}$, have the same radius $R$. Metric (265) describes the spacetime, which appears in the extremal limit of a $d$-dimensional static black hole. In the hyperbolic space $H_{2}$ we can choose a polar coordinate system $(\rho, \phi)$ with its center at point $r=R$,

$$
r=\frac{R}{\cosh \rho-\sinh \rho \cos \phi}, \quad \tau=\frac{R \sinh \rho \sin \phi}{\cosh \rho-\sinh \rho \cos \phi},
$$

(for small $\rho$ one has that $r=R+\rho \cos \phi$ and $\tau=\rho \sin \phi$ as in the polar system in flat spacetime) so that the metric takes the form

$$
d s_{\text {ext }}^{2}=R^{2}\left(d \rho^{2}+\sinh ^{2} \rho d \phi^{2}\right)+R^{2} d \omega_{d-2}^{2} .
$$

In this coordinate system the surface $\Sigma$ is defined by the condition $\rho=0$. In the entanglement entropy of a conformally-coupled scalar field the logarithmic term $s_{0}$ is conformally invariant. Therefore, it is the same [206] for the entropy of a round sphere of radius $R$ in Minkowski spacetime and in the extreme limiting geometry (267). In various dimensions $s_{0}$ can be obtained from the results $(258)-(262)$ by setting $l=l_{1}=R$. One finds $s_{0}=\frac{1}{90}$ in $d=4, s_{0}=-\frac{1}{756}$ in $d=6$ and $s_{0}=\frac{23}{113400}$ in $d=8$. For $d>4$ the logarithmic term in the entropy of a round sphere has been calculated by Casini and Huerta [38] directly in Minkowski spacetime. They have obtained $s_{0}$ in all even dimensions up to $d=14$. Subsequently, Dowker [72] has extended this result to $d=16$ and $d=18$. In arbitrary dimension $d$ the logarithmic term $s_{0}$ can be expressed in terms of Bernulli numbers as is shown in [38] and [72] 
Entanglement entropy of the extreme Reissner-Nordström black hole in $d$ dimensions. As was shown by Myers and Perry [180] a generalization of the Reissner-Nordström solution to higher dimension $d>4$ is given by Eq. (243) with

$$
g(r)=\frac{\left(r^{d-3}-r_{+}^{d-3}\right)\left(r^{d-3}-r_{-}^{d-3}\right)}{r^{2(d-3)}} .
$$

In the extreme limit $r_{+} \rightarrow r_{-}$. Expanding Eq. (268) near the horizon one finds, in this limit, that $b=(d-3)^{2} / r_{+}^{2}$. Thus, this extreme geometry is characterized by values of radii $l=r_{+} /(d-3)$ and $l_{1}=r_{+}$. In dimension $d=4$ we have $l=l_{1}$ as in the case considered above. In dimension $d>4$ the two radii are different, $l \neq l_{1}$. For the conformal coupling the values of logarithmic term $s_{0}$ in various dimensions are presented in Table 1.

Table 1: Coefficients of the logarithmic term in the entanglement entropy of an extreme ReissnerNordström black hole.

\begin{tabular}{c|ccc}
\hline$d$ & 4 & 6 & 8 \\
\hline$s_{0}$ & $\frac{1}{90}$ & $-\frac{2881}{756}$ & $\frac{1569275563}{1111320}$ \\
\hline
\end{tabular}




\section{Logarithmic Term in the Entropy of Generic Conformal Field Theory}

As we have already seen, in even dimensions, there typically appears a logarithmic term in the entanglement entropy. This term is universal in the sense that it does not depend on the scheme, which is used to regularize the UV divergences. In conformal field theories the logarithmic terms in the entropy are closely related to the conformal anomaly. In this section we discuss in detail this aspect and formulate precisely the relation between entanglement entropy and conformal anomalies.

Consider a conformal field theory in $d$ spacetime dimensions. As we have discussed throughout this review the most efficient way to calculate the corresponding entanglement entropy for a nonextremal black hole is to introduce a small angle deficit $\delta=2 \pi(1-\alpha)$ at the horizon surface $\Sigma$, compute the effective action $W\left(E^{\alpha}\right)$ on a manifold $E^{\alpha}$ with a singular surface and then apply the replica formula $S=\left.\left(\alpha \partial_{\alpha}-1\right)\right|_{\alpha=1} W\left(E^{\alpha}\right)$ and obtain from it the entanglement entropy. In $d$ dimensions the effective action has the general structure

$$
W_{\mathrm{CFT}}\left(E^{\alpha}\right)=\frac{a_{d}}{\epsilon^{d}}-\frac{a_{1}}{\epsilon^{d-2}}-. .-\frac{a_{n}}{\epsilon^{d-2 n}}-. .-a_{d / 2} \ln \epsilon+w\left(g^{(\alpha)}\right) .
$$

The logarithmic term in this expansion appears only if dimension $d$ is even. Thus, only even $d$ will be considered in this section. The terms $a_{d}, a_{d-2}, \ldots$ representing the power UV divergences, are not universal, while the term $a_{d / 2}$ is universal and is determined by the integrated conformal anomaly. The term $w\left(g^{(\alpha)}\right)$ is the UV finite part of the effective action. Under a global rescaling of the metric on $E^{\alpha}, g^{(\alpha)} \rightarrow \lambda^{2} g^{(\alpha)}$, one has

$$
w\left(\lambda^{2} g^{(\alpha)}\right)=w\left(g^{(\alpha)}\right)+a_{d / 2} \ln \lambda .
$$

An important property of the expansion (269) for a quantum field theory, which classically is conformally invariant, is that the logarithmic term $a_{d / 2}$ is conformally invariant (see [219] and references therein),

$$
a_{d / 2}\left[e^{-2 \omega} g\right]=a_{d / 2}[g] .
$$

On a manifold with a conical singularity at the surface $\Sigma$, the coefficients $a_{d-2 n}$ have a bulk part and a surface part. To first order in $(1-\alpha)$ one finds that

$$
a_{d-2 n}\left(E^{\alpha}\right)=\alpha a_{d-2 n}^{\text {bulk }}(E)+(1-\alpha) a_{d-2 n}^{\Sigma}+O(1-\alpha)^{2}
$$

For $n=d / 2$ the coefficients $a_{d / 2}^{\text {bulk }}(E)$ and $a_{d / 2}(\Sigma)$ are the integrated bulk and the surface conformal anomalies respectively. The bulk and surface terms are independently invariant under conformal transformation $g \rightarrow e^{-2 \omega} g$,

$$
a_{d / 2}^{\text {bulk }}\left(e^{-2 \omega} g\right)=a_{d / 2}^{\text {bulk }}(g) \text { and } a_{d / 2}^{\Sigma}\left(e^{-2 \omega} g\right)=a_{d / 2}^{\Sigma}(g) .
$$

Applying the replica formula one obtains the entanglement entropy

$$
\begin{aligned}
& S=\frac{s_{d-2}}{\epsilon^{d-2}}+. .+\frac{s_{d-2 n}}{\epsilon^{d-2 n}}+. .+s_{0}{ }^{\Sigma} \ln \epsilon+s(g), \\
& s_{0}=a_{d / 2}^{\Sigma}, s_{d-2 n}=a_{n}^{\Sigma}, n=1, . ., d / 2-1 \\
& s\left(\lambda^{2} g\right)=s(g)-a_{d / 2}^{\Sigma} \ln \lambda .
\end{aligned}
$$

For a regular manifold each term $a_{d-2 n}$ is the integral of a polynomial of degree $n$ in the Riemann curvature. Respectively, the surface terms $a_{d-2 n}^{\Sigma}$ are the integral over the singular surface $\Sigma$ of polynomial of degree $n-1$ in the Riemann curvature and its projections onto the subspace orthogonal to surface $\Sigma$. The concrete structure of the polynomials depends on the dimension $d$. 


\subsection{Logarithmic terms in 4-dimensional conformal field theory}

In four dimensions the bulk conformal anomaly is a combination of two terms, the topological Euler term and the square of the Weyl tensor,

$$
\begin{aligned}
a_{2}^{\text {bulk }} & =A E_{(4)}+B I_{(4)}, \\
E_{(4)} & =\frac{1}{64} \int_{E}\left(R_{\alpha \beta \mu \nu} R^{\alpha \beta \mu \nu}-4 R_{\mu \nu} R^{\mu \nu}+R^{2}\right), \\
I_{(4)} & =-\frac{1}{64} \int_{E}\left(R_{\alpha \beta \mu \nu} R^{\alpha \beta \mu \nu}-2 R_{\mu \nu} R^{\mu \nu}+\frac{1}{3} R^{2}\right) .
\end{aligned}
$$

These are, respectively, the conformal anomalies of type A and B. In a theory with $n_{s}$ particles of spin $s$, one finds [76] (the contributions of fields of spin 3/2 and 2 can be obtained from Table 2 on p. 180 of the book of Birrell and Davies [22])

$$
\begin{aligned}
& A=\frac{1}{90 \pi^{2}}\left(n_{0}+11 n_{1 / 2}+62 n_{1}+0 n_{3 / 2}+0 n_{2}\right), \\
& B=\frac{1}{30 \pi^{2}}\left(n_{0}+6 n_{1 / 2}+12 n_{1}-\frac{233}{6} n_{3 / 2}+\frac{424}{3} n_{2}\right) .
\end{aligned}
$$

The surface contribution to the conformal anomaly can be calculated directly by, for example, the heat kernel method, as in [101]. Although straightforward, the direct computation is technically involved. However, one has a short cut: there is a precise balance, observed in [196] and [112], between the bulk and surface anomalies; this balance is such that, to first order in $(1-\alpha)$, one can take $a_{2}\left(E^{\alpha}\right)=a_{2}^{\text {bulk }}\left(E^{\alpha}\right)+O(1-\alpha)^{2}$ and use for the Riemann tensor of $E^{\alpha}$ the representation as a sum of regular and singular (proportional to a delta-function concentrated on surface $\Sigma$ ) parts. The precise expressions are given in $[112,111]$. However, this representation is obtained under the assumption that the surface $\Sigma$ is a stationary point of an abelian isometry and thus has vanishing extrinsic curvature. Under this assumption, one finds that [112, 111] (see also [188])

$$
\begin{aligned}
a_{2}\left(E^{\alpha}\right) & =\alpha a_{2}^{\text {bulk }}(E)+(1-\alpha) a_{2}^{\Sigma}+O(1-\alpha)^{2}, \\
a_{2}^{\Sigma} & =A a_{A}^{\Sigma}+B a_{B}^{\Sigma}, \\
a_{A}^{\Sigma} & =\frac{\pi}{8} \int_{\Sigma}\left(R_{i j i j}-2 R_{i i}+R\right), \\
a_{B}^{\Sigma} & =-\frac{\pi}{8} \int_{\Sigma}\left(R_{i j i j}-R_{i i}+\frac{1}{3} R\right),
\end{aligned}
$$

where $R_{i j i j}=R_{\alpha \beta \mu \nu} n_{i}^{\alpha} n_{j}^{\beta} n_{i}^{\mu} n_{j}^{\nu}, R_{i i}=R_{\alpha \beta} n_{i}^{\alpha} n_{i}^{\beta}$.

Each surface term in Eq. (276) is invariant under a sub-class of conformal transformations, $g \rightarrow e^{-2 \omega} g$, such that the normal derivatives of $\omega$ vanish on surface $\Sigma$. Moreover, the surface term due to the bulk Euler number is a topological invariant: using the Gauss-Codazzi equation

$$
R=R_{\Sigma}+2 R_{i i}-R_{i j i j}-k^{i} k^{i}+\operatorname{Tr} k^{2},
$$

where $R_{\Sigma}$ is the intrinsic Ricci scalar of the surface and $k_{\alpha \beta}^{i}$ is the extrinsic curvature, and in the assumption of vanishing extrinsic curvature the $a_{A}^{\Sigma}$ term, as shown in [111], is proportional to the Euler number of the $2 \mathrm{D}$ surface $\Sigma$,

$$
a_{A}^{\Sigma}=\frac{\pi}{8} \int_{\Sigma} R_{\Sigma}
$$

where $R_{\Sigma}$ is the intrinsic curvature of $\Sigma$. 
For completeness we note that this result can be generalized to an arbitrary codimension 2 surface in 4-dimensional spacetime. Then, the conformal transformation is generalized to any function $\omega$ with non-vanishing normal derivative at $\Sigma$. The terms with the normal derivatives of $\omega$ in the conformal transformation of $a_{2}^{\Sigma}$ can be canceled by adding the quadratic combinations of extrinsic curvature, $\operatorname{Tr} k^{2}$ and $k_{a} k_{a}$. The analysis presented by Solodukhin [204] (this analysis is based on an earlier consideration by Dowker [70]) results in the following expressions

$$
\begin{aligned}
& a_{2}^{\Sigma}=A a_{A}^{\Sigma}+B a_{B}^{\Sigma}, \\
& a_{A}^{\Sigma}=\frac{\pi}{8} \int_{\Sigma}\left(R_{i j i j}-2 R_{i i}+R-\operatorname{Tr} k^{2}+k_{i} k_{i}\right)=\frac{\pi}{8} \int_{\Sigma} R_{\Sigma}, \\
& a_{B}^{\Sigma}=-\frac{\pi}{8} \int_{\Sigma}\left(R_{i j i j}-R_{i i}+\frac{1}{3} R-\left(\operatorname{Tr} k^{2}-\frac{1}{2} k_{i} k_{i}\right)\right) .
\end{aligned}
$$

This is the most general form of the logarithmic term in the entanglement entropy in four spacetime dimensions.

Thus, as follows from Eq. (274), the logarithmic term in the entanglement entropy of a black hole in four dimensions is

$$
s_{0}^{(d=4)}=A \frac{\pi}{8} \int_{\Sigma} R_{\Sigma}-B \frac{\pi}{8} \int_{\Sigma}\left(R_{i j i j}-R_{i i}+\frac{1}{3} R\right) .
$$

For conformal fields of various spin the values of $A$ and $B$ are presented in Eq. (275).

Consider some particular examples.

Extreme static geometry. For an extreme geometry, which has the structure of the product $H_{2} \times S_{2}$ and characterized by two dimensionful parameters $l$ (radius of $H_{2}$ ) and $l_{1}$ (radius of $S_{2}$ ), the logarithmic term in the entropy

$$
s_{0}^{\mathrm{ext}}=A \pi^{2}-\frac{B \pi^{2}}{3}\left(1-\frac{l_{1}^{2}}{l^{2}}\right)
$$

is determined by both the anomalies of type A and B. In the case of the extreme ReissnerNordström black hole one has $l=l_{1}$ and the logarithmic term (281) is determined only by the anomaly of type A. For a conformal scalar field one has that $A=B / 3=1 / 90 \pi^{2}$ and this equation reduces to Eq. (258). As we already discussed, the geometry $H_{2} \times S_{2}$ for $l=l_{1}$ is conformal to flat 4-dimensional space. Thus, the Weyl tensor vanishes in this case as does its projection to the subspace orthogonal to horizon $S_{2}$. That is why the type B anomaly does not contribute in this case to the logarithmic term.

The Schwarzschild black hole. In this case, the background is Ricci flat and the logarithmic term is determined by the difference of $A$ and $B$,

$$
s_{0}^{\mathrm{Sch}}=(A-B) \pi^{2} .
$$

The same is true for any Ricci flat metric. For a conformal scalar field, Eq. (282) reduces to Eq. (88). For a scalar field the relation of the logarithmic term in the entropy and the conformal anomaly was discussed by Fursaev [103]. The logarithmic term vanishes if $A=B$. In this case the Riemann tensor does not appear in the conformal anomaly (274) so that the anomaly vanishes if the metric is Ricci flat. In particular, the relation $A=B$ can be found from the $\mathcal{N}=4$ superconformal gauge theory, dual to supergravity on $\mathrm{AdS}_{5}$, according to the AdS/CFT correspondence of Maldacena [167]. The conformal anomaly in this theory was calculated in [131]. 
Non-extreme and extreme Kerr black hole. For a Kerr black hole (characterized by mass $m$ and rotation $a$ ) the logarithmic term does not depend on the parameter of the rotation and it takes the same form

$$
s_{0}^{\mathrm{Kerr}}=(A-B) \pi^{2}
$$

as in the case of the Schwarschild metric. In the extreme limit $a=m$ the logarithmic term takes the same value as (283).

\subsection{Logarithmic terms in 6-dimensional conformal field theory}

In six dimensions, omitting the total derivative terms, the conformal anomaly is a combination of four different conformal invariants [17]

$$
a_{3}^{\mathrm{bulk}}=\int_{M^{6}}\left(B_{1} I_{1}+B_{2} I_{2}+B_{3} I_{3}+A E_{6}\right)
$$

where $I_{1}, I_{2}$ and $I_{3}$ are cubic in the Weyl tensor

$$
\begin{aligned}
& I_{1}=C_{k i j l} C^{i m n j} C_{m n}^{k l}, \quad I_{2}=C_{i j}{ }^{k l} C_{k l}{ }^{m n} C_{m n}{ }^{i j}, \\
& I_{3}=C_{i k l m}\left(\nabla^{2} \delta_{j}^{i}+4 R_{j}^{i}-\frac{6}{5} R \delta_{j}^{i}\right) C^{j k l m},
\end{aligned}
$$

and $E_{6}$ is the Euler density (60)

$$
E_{6}=\frac{1}{3072 \pi^{3}} \epsilon_{\mu_{1} \mu_{2} \ldots \mu_{6}} \epsilon^{\nu_{1} \nu_{2} \ldots \nu_{6}} R_{\nu_{1} \nu_{2}}^{\mu_{1} \mu_{2}} \ldots R_{\nu_{5} \nu_{6}}^{\mu_{5} \mu_{6}}
$$

As was shown in [17] in a free conformal field theory with $n_{0}$ scalars, $n_{1 / 2}$ Dirac fermions and $n_{B}$ 2 -form fields, one has that ${ }^{10}$

$$
\begin{aligned}
A & =\frac{8 \cdot 3 !}{7 !}\left(-\frac{5}{72} n_{0}-\frac{191}{72} n_{1 / 2}-\frac{221}{4} n_{B}\right), \\
B_{1} & =\frac{1}{(4 \pi)^{3} 7 !}\left(\frac{28}{3} n_{0}+\frac{896}{3} n_{1 / 2}+\frac{8008}{3} n_{B}\right), \\
B_{2} & =\frac{1}{(4 \pi)^{3} 7 !}\left(-\frac{5}{3} n_{0}+32 n_{1 / 2}+\frac{2378}{3} n_{B}\right), \\
B_{3} & =\frac{1}{(4 \pi)^{3} 7 !}\left(-2 n_{0}-40 n_{1 / 2}-180 n_{B}\right) .
\end{aligned}
$$

Applying the formulas (55) to $I_{1}, I_{2}$ and $I_{3}$ and using the relation (62) for the Euler number, one finds for the logarithmic term in the entanglement entropy of 4-dimensional surface $\Sigma$ in a 6-dimensional conformal field theory

$$
s_{0}^{d=6}=B_{1} s_{B 1}+B_{2} s_{B 2}+B_{3} s_{B 3}+A s_{A},
$$

where

$$
s_{A}=\chi[\Sigma]
$$

\footnotetext{
${ }^{10}$ Note that the coefficient $b_{6}$ of [17] is related to $a_{3}$ as $b_{6}=-a_{3}$.
} 
is the Euler number of the surface $\Sigma$, and

$$
\begin{aligned}
& s_{B 1}=6 \pi\left(C^{j \alpha \beta i} C_{\alpha \beta}^{i j}-C^{j \alpha \beta j} C_{\alpha \beta}^{i i}-\frac{1}{4} C^{i \alpha \beta \mu} C_{\alpha \beta \mu}^{i}+\frac{1}{20} C^{\alpha \beta \mu \nu} C_{\alpha \beta \mu \nu}\right), \\
& s_{B 2}=6 \pi\left(2 C^{i j \alpha \beta} C_{\alpha \beta}^{i j}-C^{i \alpha \beta \mu} C_{\alpha \beta \mu}^{i}+\frac{1}{5} C^{\alpha \beta \mu \nu} C_{\alpha \beta \mu \nu}\right), \\
& s_{B 3}=8 \pi\left(\nabla^{2} C^{i j i j}+4 R_{\alpha}^{i} C^{\alpha j i j}-R_{\alpha \beta} C^{\alpha i \beta i}-\frac{6}{5} R C^{i j i j}+C_{\alpha \beta \mu}^{i} C^{i \alpha \beta \mu}-\frac{3}{5} C^{\alpha \beta \mu \nu} C_{\alpha \beta \mu \nu}\right)
\end{aligned}
$$

where tensors with Latin indices are obtained by contraction with components of normal vectors $n_{\alpha}^{i}, i=1,2$. Note that in Eq. (295) we used for brevity the notation $\nabla^{2} C^{i j i j} \equiv n_{\alpha}^{i} n_{\beta}^{j} n_{\mu}^{i} n_{\nu}^{j} \nabla^{2} C^{\alpha \beta \mu \nu}$. Eqs. (293), (294), and (295) agree with the results obtained in [134].

Let us consider some examples.

6-dimensional Schwarzschild black hole. The 6-dimensional generalization of the Schwarzschild solution is [180]

$$
d s^{2}=g(r) d \tau^{2}+g^{-1}(r) d r^{2}+r^{2} d \omega_{S_{4}}^{2}, \quad g(r)=1-\frac{r_{+}^{3}}{r^{3}}
$$

where $d \omega_{S_{4}}^{2}$ is a metric of unit 4-sphere. The area of horizon is $A_{+}=\frac{8 \pi^{2}}{3} r_{+}^{4}$. The Euler number of the horizon $\chi\left[S_{4}\right]=2$. This metric is Ricci flat so that only the Riemann tensor contributes to the Weyl tensor. The logarithmic term in this case is

$$
s_{0}^{\mathrm{Sch}}=16 \pi^{3}\left(-51 B_{1}+156 B_{2}-192 B_{3}\right)+2 A .
$$

It is interesting to note that this term vanishes in the case of the interacting $(2,0)$ conformal theory, which is dual to supergravity on $\mathrm{AdS}_{7}$. Indeed, in this case one has $[131,17]$

$$
\begin{aligned}
& B_{i}=\frac{b_{i}}{(4 \pi)^{3} 7 !}, A=\frac{8 \cdot 3 !}{7 !} a \\
& a=-\frac{35}{2}, b_{1}=1680, b_{2}=420, b_{3}=-140
\end{aligned}
$$

so that $s_{0}^{(2,0)}=0$. This is as expected. The Riemann tensor does not appear in the conformal anomaly of the strongly interacting $(2,0)$ theory so that the anomaly identically vanishes if the spacetime is Ricci flat. This property is not valid in the case of the free $(2,0)$ tensor multiplet [17] so that the logarithmic term of the free multiplet is non-vanishing.

Conformally-flat extreme geometry. In conformally-flat spacetime the Weyl tensor $C_{\alpha \beta \mu \nu}=$ 0 so that terms (293), (294) and (295) identically vanish. The logarithmic term (291) then is determined by the anomaly of type A only. In particular, this is the case for the extreme geometry $H_{2} \times S_{4}$ with equal radii $l=l_{1}$ of two components. One has

$$
s_{0}^{\mathrm{ext}}=2 A
$$

for this extreme geometry. This geometry is conformal to flat spacetime and the logarithmic term (299) is the same as for the entanglement entropy of a round sphere in flat 6-dimensional spacetime. This generalizes the result discussed in Section 5.3.2 for the entropy of a round sphere due to a conformal scalar field. The result (299), as is shown in [39, 181], generalizes to a spherical entangling surface in a conformally-flat spacetime of any even dimension. 


\subsection{Why might logarithmic terms in the entropy be interesting?}

By a logarithmic term we mean both the logarithmically UV-divergent term in the entropy and the UV-finite term, which scales logarithmically with respect to the characteristic size of the black hole. As we have seen, these terms are identical. However, after the UV divergences in the entropy have been renormalized, it is the UV finite term, which scales logarithmically, that will interest us here.

i) First of all, the logarithmic terms are universal and do not depend on the way the entropy was calculated and on the scheme in which the UV divergences have been regularized. This is in contrast to the power UV divergences in the entropy that depend both on the calculation procedure and on the regularization scheme.

ii) The logarithmic terms are related to the conformal anomalies. As the conformal anomalies play an important role in the modern theoretical models, any new manifestation of the anomalies merits our special attention. This may be even more important in view of ideas that the conformal symmetry may play a more fundamental role in nature than is usually thought. As is advocated by 't Hooft in a number of recent papers [217, 218, 216] a crucial ingredient for understanding Hawking radiation and entropy is to realize that gravity itself is a spontaneously-broken conformal theory.

iii) For a large class of extremal black-hole solutions, which arise in supergravity theories considered as low energy approximations of string theory, there exists a microscopic calculation of the entropy. This calculation requires a certain amount of unbroken supersymmetry, so that the black holes in question are the Bogomol'nyi-Prasad-Sommerfeld (BPS) type solutions and use the conformal field theory tools, such as the Cardy formula. The Cardy formula predicts certain logarithmic corrections to the entropy (these corrections are discussed, in particular, in [35] and [201]). One may worry whether exactly the same corrections are reproduced in the macroscopic, field theoretical, computation of the entropy. This aspect was studied recently in [8] for black holes in $\mathcal{N}=4$ supergravity and at least some partial (for the entropy due to matter multiplet of the supergravity) agreement with the microscopic calculation has indeed been observed.

iv) Speaking about the already renormalized entropy of black holes and taking into account the backreaction of the quantum matter on the geometry, the black-hole entropy can be represented as a series expansion in powers of Newton's constant, $G / r_{g}^{2}$ (the quantity $1 / r_{g}^{2}$, where $r_{g}=2 G M$ is the size of a black hole, is the scale of the curvature at the horizon; thus, the ratio $G / r_{g}^{2}$ measures the strength of gravitational self-interaction at the horizon) or, equivalently, in powers of $M_{\mathrm{PL}}^{2} / M^{2}$. In particular, for the Schwarzschild black hole of mass $M$ in four spacetime dimensions, one finds

$$
S=4 \pi \frac{M^{2}}{M_{\mathrm{PL}}^{2}}+\sigma \ln M+O\left(\frac{M_{\mathrm{PL}}^{2}}{M^{2}}\right) .
$$

The logarithmic term is the only correction to the classical Bekenstein-Hawking entropy that is growing with mass $M$.

v) Although the logarithmic term is still negligibly small compared to the classical entropy for macroscopic black holes, it becomes important for small black holes especially at the latest stage of black hole evaporation. In particular, it manifests itself in a modification of the Hawking temperature as a function of mass $M[103]$. Indeed, neglecting the terms $O\left(\frac{M_{\mathrm{PL}}^{2}}{2}\right)$ in the entropy $(300)$ one finds

$$
1 / T_{H}=\frac{\partial S}{\partial M}=8 \pi M / M_{\mathrm{PL}}^{2}+\sigma M^{-1}
$$

so that the Hawking temperature $T_{H} \sim M$ for small black holes. Depending on the sign of the coefficient $\sigma$ in Eq. (300) there can be two different scenarios. If $\sigma<0$, then the entropy $S(M)$ as 
function of mass develops a minimum at some value of $M_{\min } \sim M_{\mathrm{PL}}$. For this value of the mass the temperature (301) becomes infinite. This is the final point (at least in this approximation) of the evaporation for black holes of mass $M>M_{\min }$. It is reached in finite time. Not worrying about exact numerical factors one has

$$
\frac{d M}{d t} \sim-T_{H}^{4} A_{+} \sim-T_{H}^{4} M^{2}
$$

for the evaporation rate. For large black holes $\left(M_{0} \gg M_{\min }\right)$ the evaporation time is $t_{\mathrm{BH}} \sim$ $M_{0}^{3} / M_{\mathrm{PL}}^{4}$. This evaporation time can be obtained by solving Eq. (302) with the classical expression for the Hawking temperature $T_{H} \sim M_{\mathrm{PL}}^{2} / M$, i.e., without the correction as in Eq. (301). Thus, if there is no logarithmic term in Eq. (300), any black hole evaporates in finite time. If the correction term is present, it becomes important for $M_{0} \sim M_{\min }$. Then, assuming that $M_{\min } \sim M_{\mathrm{PL}}$, one finds $t_{\mathrm{BH}} \sim\left(M_{0}-M_{\mathrm{PL}}\right)^{5} / M_{\mathrm{PL}}^{4}$ for the evaporation time. On the other hand, a black hole of mass $M_{0}<M_{\text {min }}$, if it exists, evaporates down to zero mass in infinite time. Similar behavior is valid for black holes of arbitrary mass $M_{0}$ if $\sigma>0$ in Eqs. (300) and (301). The evaporation rate considerably slows down for small black holes since the Hawking temperature $T_{H} \sim M$ for small $M$. The black hole then evaporates to zero mass in infinite time. Asymptotically, for large time $t$, the mass of the black hole decreases as $M(t) \sim t^{-1 / 5}$. Thus, the sub-Planckian black holes if $(\sigma<0)$ and any black holes if $(\sigma>0)$ live much longer than one would have expected if one used the classical expression for the entropy and for the Hawking temperature. 


\section{A Holographic Description of the Entanglement Entropy of Black Holes}

A popular trend in modern fundamental physics is to reconsider various, sometimes very well known, phenomena from the point of view of holography. Holography is a rather general statement that the physics inside a spatial region can be understood by looking at a certain theory defined on the boundary of the region. This holographic principle was first formulated by 't Hooft [215] and later generalized by Susskind [212]. For a review of the holographic principle see [24]. A concrete realization of holography is the AdS/CFT correspondence [167, 223, 125]. According to this correspondence the theory of supegravity (more precisely string theory, the low energy regime of which is described by supegravity) in a $(d+1)$-dimensional anti-de Sitter spacetime (AdS) is equivalent to a quantum conformal field theory (CFT) defined on a $d$-dimensional boundary of the anti-de Sitter. There is a precise dictionary definition of how phenomena on one side of the correspondence can be translated into phenomena on the other side. The correspondence has proven to be extremely useful, both for better understanding gravitational physics and quantum field theory. If $d=4$, then the CFT on the boundary is known to be an $\mathcal{N}=4$ superconformal gauge theory. This theory is strongly coupled and in many aspects resembles the QCD. Thus utilizing the correspondence one, in particular, may gain some information on how theories of this type behave (for a review on the correspondence and its applications see [1]).

One of the aspects of the AdS/CFT correspondence is geometrical. The boundary theory provides certain boundary conditions for the gravitational theory in bulk so that one may decode the hologram: reconstruct the bulk spacetime from the boundary data. As was analyzed in [60], for this reconstruction, the boundary data one has to specify consist of the boundary metric and the vacuum expectation of the stress-energy tensor of the boundary CFT. The details are presented in [60]; see also [193].

Entanglement entropy is one of the fundamental quantities, which characterize the boundary theory. One would think that it should have an interpretation within the AdS/CFT correspondence. This interpretation was suggested in 2006 by Ryu and Takayanagi [189, 188] (for a review on this proposal see [185]). This proposal is very interesting since it allows one to compute the entanglement entropy in a purely geometrical way (see also [109]).

\subsection{Holographic proposal for entanglement entropy}

Let $M$ be a $(d+1)$-dimensional asymptotically anti-de Sitter spacetime. Its conformal boundary is a $d$-dimensional spacetime $\partial M$. On a slice of constant time $t$ in $\partial M$ one picks a closed $(d-2)$ dimensional surface $\Sigma$ and defines the entropy of entanglement with respect to $\Sigma$. Now, on the constant $t$ slice of the $(d+1)$-dimensional anti-de Sitter spacetime, consider a $(d-1)$-dimensional minimal surface $\gamma$ such that its boundary in $\partial M$ is the surface $\Sigma, \partial \gamma=\Sigma$. According to [189, 188], the following quantity

$$
S=\frac{A(\gamma)}{4 G_{d+1}}
$$

where $A(\gamma)$ is the area of minimal surface $\gamma$ and $G_{d+1}$ is Newton's constant in the $(d+1)$-dimensional gravitational theory, is equal to the entanglement entropy one has calculated in the boundary conformal field theory. This holographic proposal has passed many tests and never failed. It has correctly reproduced the entropy in all cases where it is explicitly known. In particular, in two spacetime dimensions $(d=2)$ it correctly reproduced (10) and (11) for the entropy at finite size and at finite entropy respectively. In higher dimensions $(d>2)$ the area of minimal surface $\gamma$ diverges when it is extended till $\partial M$. This is an important feature, typical of the AdS/CFT correspondence. In fact, instead of a conformal boundary $\partial M$, one has to consider a regularized

Living Reviews in Relativity

http: //www. livingreviews.org/lrr-2011-8 
boundary $\partial M_{\epsilon}$ located at a small distance $\epsilon$ (measured in terms of some radial coordinate $\rho$ ). In the AdS/CFT correspondence the divergence in $\epsilon$ has the interpretation of a UV divergence in the boundary quantum field theory. Considering the regularized surface $\gamma$, which extends to $\partial M_{\epsilon}$, one finds that its area, to leading order in $\epsilon$, behaves as $A(\gamma) \sim A(\Sigma) / \epsilon^{d-2}$. Taking this behavior of the area, one sees that the proposal (303) correctly reproduces the proportionality of the entropy to the area of surface $\Sigma$ and its dependence on the UV cutoff $\epsilon$.

However, we note that in certain situations the choice of the minimal surface $\Sigma$ may not be unique. In particular, if the dividing surface $\Sigma$ has several components or if the quantum field resides inside a cavity instead of being defined on an infinite space, there is more than one natural choice of the minimal surface $\gamma$. Different choices may correspond to different phases in the boundary theory [157].

\subsection{Proposals for the holographic entanglement entropy of black holes}

If one wants to generalize the proposal of Ryu and Takayanagi to black holes, the first step would be to find a $(d+1)$-dimensional asymptotically-AdS metric, which solves the Einstein equations in bulk and whose conformal boundary describes a $d$-dimensional black hole. To find such a metric explicitly may be a difficult task, although some exact solutions are known. First of all, it is easy to construct an AdS metric, which gives a de Sitter spacetime on the boundary. The de Sitter horizon is in many aspects, similar to a black hole horizon. Entanglement entropy associated to the de Sitter horizon [130, 139] has the same properties as the entropy of any other Killing horizon. In four dimensions $(d=3)$ an exact solution to the Einstein equations has been found in [84] that describes a 3D black hole on the boundary. In three dimensions $(d=2)$, an exact solution, which describes a generic static two-dimensional black hole on the boundary, has been found in [194]. On the other hand, the results of [60] show that for any chosen metric on the boundary $\partial M$, one can find, at least in a small region close to the boundary, an exact solution to the Einstein equations with negative cosmological constant. Exact formulas are given in [60]. Thus, at least principally, it is not a problem to find an asymptotically AdS metric which describes a black hole on the boundary.

The next question is how to choose the minimal surface $\gamma$. Emparan's proposal [83] consists of the following. Suppose the metric on the boundary of asymptotically-AdS spacetime describes a black hole with a Killing horizon at surface $\Sigma$. Presumably, the horizon on the boundary $\partial M$ is extended to the bulk. The bulk horizon is characterized by vanishing extrinsic curvature and is a minimal $(d-1)$-dimensional surface. Thus, one can choose the bulk horizon to be that minimal surface $\gamma$, the area of which should appear in the holographic formula (303). In this construction the Killing horizon $\Sigma$ is the only boundary of the minimal surface $\gamma$. This prescription is perfectly eligible if one computes the entanglement entropy of a black hole in infinite spacetime. In [83] it was applied to the entropy of a black hole residing on the 2-brane in the 4d solution of [84]. In [139] a similar prescription is used to compute entanglement entropy of the de Sitter horizon.

On the other hand, in certain situations it is interesting to consider a black hole residing inside a cavity, the "black hole in a box". Then, as we have learned in the two-dimensional case, the entanglement entropy will depend on the size $L$ of the box so that, in the limit of large $L$, the entropy will have a thermal contribution proportional to the volume of the box. This contribution is additional to the pure entanglement part, which is due to the presence of the horizon $\Sigma$. In order to reproduce this dependence on the size of the box, one should use a different proposal. A relevant proposal was suggested by Solodukhin in [202].

Let a $d$-dimensional spherically-symmetric static black hole with horizon $\Sigma$ lie on the regularized boundary (with regularization parameter $\epsilon$ ) of asymptotically anti-de Sitter spacetime $\operatorname{AdS}_{d+1}$ inside a spherical cavity $\Sigma_{L}$ of radius $L$. Consider a minimal d-surface $\Gamma$, whose boundary is the union of $\Sigma$ and $\Sigma_{L}$. $\Gamma$ has saddle points, where the radial AdS coordinate has an extremum. 
By spherical symmetry the saddle points form a $(d-2)$-surface $\mathcal{C}$ with the geometry of a sphere. Consider the subset $\Gamma_{h}$ of $\Gamma$ whose boundary is the union of $\Sigma$ and $\mathcal{C}$. According to the prescription of [202], the quantity

$$
S=\frac{\operatorname{Area}\left(\Gamma_{h}\right)}{4 G_{N}^{d+1}}
$$

is equal to the entanglement entropy of the black hole in the boundary of AdS. In particular, it gives the expected dependence of the entropy on the UV regulator $\epsilon$. The minimal surface $\Gamma_{h}$ "knows" about the existence of the other boundary $\Sigma_{L}$. That is why Eq. (304) reproduces correctly the dependence of the entropy on the size of the "box". In [202] this proposal has been verified for $d=2$ and $d=4$.

It should be noted that as far as the UV divergent part of the entanglement entropy is concerned, the two proposals [83, 139] and [202] give the same result. This is due to the fact that the UV divergences come from that part of the minimal surface, which approaches the boundary $\partial M$ of the AdS spacetime. In both proposals this part of the surfaces $\gamma$ and $\Gamma_{h}$ is the same. Thus, the difference is in the finite terms, which are due to global properties of the minimal surface.

From the geometrical point of view, the holographic calculation of the logarithmic term in the entanglement entropy is related to the surface anomalies studied by Graham and Witten [123] (this point is discussed in [191]).

\subsection{The holographic entanglement entropy of 2D black holes}

In order to check the proposal (304) one needs a solution to the bulk Einstein equations that describes a black hole on the boundary of AdS. In three dimensions a solution of this type is known explicitly [194],

$$
d s^{2}=\frac{d \rho^{2}}{4 \rho^{2}}+\frac{1}{\rho}\left[f(x)\left(1+\frac{1}{16} \frac{f^{\prime 2}-b^{2}}{f} \rho\right)^{2} d \tau^{2}+\frac{1}{f(x)}\left(1+\frac{1}{4} f^{\prime \prime} \rho-\frac{1}{16} \frac{f^{\prime 2}-b^{2}}{f} \rho\right)^{2} d x^{2}\right]
$$

where the AdS radius is set to unity. At asymptotic infinity $(\rho=0)$ of the metric (305) one has the $2 \mathrm{D}$ black hole metric

$$
d s_{2 d}^{2}=f(x) d \tau^{2}+f^{-1}(x) d x^{2}
$$

where $f(x)$ has a simple zero in $x=x_{+}$. The cavity $\Sigma_{L}$ is placed at $x=L$ so that $x_{+} \leq x \leq L$. The regularity of the metric (306) at the horizon $x=x_{+}$requires that $0 \leq \tau \leq \beta_{H}, \beta_{H}=4 \pi / f^{\prime}\left(x_{+}\right)$. Note that Eq. (305) is a vacuum solution of the Einstein equations for any function $f(x)$. The regularity of the $3 \mathrm{D}$ metric (305) requires that the constant $b$ should be related to the Hawking temperature of the two-dimensional horizon by $b=f^{\prime}\left(x_{+}\right)$. The geodesic $\Gamma$ lies in the hypersurface of constant time $\tau$. The induced metric on the hypersurface $(\rho, x)$ has a constant curvature equal to -2 for any function $f(x)$ and is, thus, related by a coordinate transformation to the metric

$$
d s_{\tau}^{2}=\frac{d r^{2}}{4 r^{2}}+\frac{1}{r} d w^{2}
$$

The exact relation between the two coordinate systems is

$$
\begin{aligned}
w & =\frac{1}{8 b} e^{z(x)}\left(\frac{16 f(x)-\left(b^{2}-f_{x}^{\prime 2}\right) \rho}{16 f(x)+\left(b-f_{x}^{\prime}\right)^{2} \rho}\right), \\
r & =f(x) e^{2 z(x)} \frac{\rho}{\left(16 f(x)+\left(b-f_{x}^{\prime}\right)^{2} \rho\right)^{2}},
\end{aligned}
$$


where $z(x)=\frac{b}{2} \int_{L}^{x} \frac{d x}{f(x)}$. The equation for the geodesic in the metric (307) is $r=\frac{1}{C^{2}}-\left(w-w_{0}\right)^{2}$. The geodesic length between two points lying on the geodesic with radial coordinates $r_{1}$ and $r_{2}$ is

$$
\gamma(1,2)=\frac{1}{2}\left(\ln \left[\frac{1-\sqrt{1-C^{2} r}}{1+\sqrt{1-C^{2} r}}\right]\right)_{r_{1}}^{r_{2}} .
$$

The saddle point of the geodesic is at $r_{m}=1 / C^{2}$. The constant $C$ is determined from the condition that the geodesic $\Gamma$ joins points $\left(x=x_{+}, \rho=\epsilon^{2}\right)$ and $\left(x=L, \rho=\epsilon^{2}\right)$ lying on the regularized (with regularization parameter $\epsilon$ ) boundary. In the limit of small $\epsilon$ one finds that

$$
\frac{C^{2} r_{+}}{4}=\epsilon^{2} \frac{b^{2}}{f\left(x_{+}\right)} \exp \left(-b \int_{x_{+}}^{L} \frac{d x}{f(x)}\right) .
$$

The length of the geodesic $\Gamma_{h}$ joining point $r_{+}$corresponding to $\left(x=x_{+}, \rho=\epsilon\right)$ and thus the saddle point is $\gamma\left(\Gamma_{h}\right)=-\frac{1}{2} \ln \frac{C^{2} r_{+}}{4}$.

Now, one has to take into account that, in the AdS/CFT correspondence, the value of Newton's constant in the bulk is related to the number of quantum fields living in the boundary $\partial M$. In the $\mathrm{AdS}_{3} / \mathrm{CFT}_{2}$ case, one has that $\frac{1}{G_{N}}=\frac{2 c}{3}$, where $c$ is the central charge of boundary CFT. Thus, one finds that the holographic entropy (304)

$$
\begin{aligned}
S & =\frac{1}{4 G_{N}} \gamma\left(\Gamma_{h}\right) \\
& =\frac{c}{6} \ln \frac{1}{\epsilon}+\frac{c}{12}\left[\int_{x_{+}}^{L} \frac{d x}{f(x)}\left(b-f^{\prime}\right)+\ln f(L)-\ln b^{2}\right],
\end{aligned}
$$

where $b=f^{\prime}\left(x_{+}\right)$, indeed coincides with expression (219) for the holographic entanglement entropy of the 2D black hole in conformal field theory. In particular, for large values of $L$ the holographic formula for the entropy correctly reproduces the entropy of thermal gas $S_{t h}=\frac{c \pi}{3} T_{H} L$ (we remember that $\left.T_{H}=f^{\prime}\left(x_{+}\right) / 4 \pi\right)$. This is a consequence of the choice of the minimal surface $\Gamma_{h}$ made in the proposal (304).

\subsection{Holographic entanglement entropy of higher dimensional black holes}

In higher dimensions there is no known exact solution similar to Eq. (305). However, a solution in the form of a series expansion in $\rho$ is available. In the rest of this section we focus on the case of boundary dimension 4 . Then one finds [131]

$$
\begin{aligned}
& d s^{2}=\frac{d \rho^{2}}{4 \rho^{2}}+\frac{1}{\rho} g_{i j}(x, \rho) d x^{i} d x^{j} \\
& g(x, \rho)=g_{(0)}(x)+g_{(2)} \rho+g_{(4)} \rho^{2}+h_{(4)} \rho^{2} \ln \rho+. .
\end{aligned}
$$

where $g_{(0) i j}(x)$ is the boundary metric and coefficient [131]

$$
g_{(2) i j}=-\frac{1}{2}\left(R_{i j}-\frac{1}{6} R g_{(0) i j}\right)
$$

is the local covariant function of the boundary metric. Coefficient $g_{(4)}$ is not expressed as a local function of the boundary metric and is related to the stress-energy tensor of the boundary CFT [60], which has an essentially non-local nature. $h_{(4)}$ is a local, covariant, function of the boundary metric and is obtained as a variation of the integrated conformal anomaly with respect to the boundary metric [60]. Its explicit form was computed in [60]. $h_{(4)}$ is a traceless tensor and in mathematics 
literature it is known as the "obstruction" tensor [122]. The explicit form of $h_{(4)}$ or $g_{(4)}$ is not important if one wants to compute the UV divergence terms in the entropy.

One may choose the boundary metric describing a static spherically-symmetric black hole to take the form

$$
d s^{2}=f(r) d \tau^{2}+f^{-1}(r) d r^{2}+r^{2}\left(d \theta^{2}+\sin ^{2} \theta d \phi^{2}\right) .
$$

The minimal surface $\Gamma$ lies in the hypersurface of the constant $\tau$ of 5 -dimensional spacetime (310). The induced metric on the hypersurface takes the form

$$
d s_{\tau}^{2}=\frac{d \rho^{2}}{4 \rho^{2}}+\frac{1}{\rho}\left[F \frac{d r^{2}}{f(r)}+R^{2}\left(d \theta^{2}+\sin ^{2} \theta d \phi^{2}\right)\right],
$$

where functions $F(r, \rho)=g_{(0)}^{r r} g_{r r}$ and $R^{2}(r, \rho)=g_{\theta \theta}$ have a $\rho$-expansion due to Eq. (310). The minimal surface $\Gamma$ can be parameterized by $(\rho, \theta, \phi)$. Instead of the radial coordinate $r$, it is convenient to introduce the coordinate $y=\int d r / \sqrt{f}$ so that, near the horizon, one has $r=$ $r_{+}+b y^{2} / 4+O\left(y^{4}\right)$. The coordinate $y$ measures the invariant distance along the radial direction. By spherical symmetry, the area to be minimized is

$$
\operatorname{Area}(\Gamma)=4 \pi \int \frac{d \rho}{\rho} R^{2} \sqrt{\frac{1}{4 \rho^{2}}+\frac{F}{\rho}\left(\frac{d y}{d \rho}\right)^{2}},
$$

where $\rho_{m}$ is the saddle point. In the vicinity of the horizon $(y \ll 1)$, we can neglect the dependence of the functions $F(y, \rho)$ and $R^{2}(y, \rho)$ on the coordinate $y$. The minimization of the area of the surface gives the equation

$$
\frac{F R^{2} \frac{d y}{d \rho}}{\rho^{2} \sqrt{\frac{1}{4 \rho^{2}}+\frac{F}{\rho}\left(\frac{d y}{d \rho}\right)^{2}}}=C=\text { const. }
$$

The area of the minimal surface $\Gamma_{h}$ is then given by the integral

$$
\operatorname{Area}\left(\Gamma_{h}\right)=2 \pi \int_{\epsilon^{2}}^{\rho_{m}} d \rho \mathcal{A}(\rho), \mathcal{A}=\frac{R^{2}}{\rho^{2} \sqrt{1-\frac{C^{2} \rho^{3}}{F R^{4}}}} .
$$

Using Eq. (310) we find that $\mathcal{A}(\rho)=\left[\frac{r_{+}^{2}}{\rho^{2}}+\frac{g_{(2) \theta \theta}\left(r_{+}\right)}{\rho}+..\right]$. Substituting this expansion into Eq. (316) we find that the first two terms produce divergences (when $\epsilon$ goes to zero), which, according to our proposal, are to be interpreted as UV divergences of the entanglement entropy. At the black hole horizon, one has the relation $\left.2 R_{\theta \theta}\right|_{r_{+}}=r_{+}^{2}\left(R-R_{a a}\right)$. Putting everything together and applying proposal (304), one finds for the divergent part

$$
S_{\mathrm{div}}=\frac{A(\Sigma)}{4 \pi \epsilon^{2}} N^{2}-\frac{N^{2}}{2 \pi} \int_{\Sigma}\left(\frac{1}{4} R_{a a}-\frac{1}{6} R\right) \ln \epsilon,
$$

where $A(\Sigma)=4 \pi r_{+}^{2}$ is the horizon area.

The logarithmic term in Eq. (317) is related to the logarithmic divergence as (calculated holographically in [131])

$$
W_{\log }=\frac{N^{2}}{4 \pi^{2}} \int\left(\frac{1}{8} R_{\mu \nu}^{2}-\frac{1}{24} R^{2}\right) \ln \epsilon
$$

in the quantum effective action of boundary CFT. This relation is a particular manifestation of the general formula (280) that relates the logarithmic term in the entropy to the conformal anomalies 
of type A and B. One notes that in the $\mathcal{N}=4$ superconformal $S U(N)$ gauge theory one has that $A=B=\frac{N^{2}}{\pi^{2}}$.

It should be noted that the UV finite terms and their dependence on the size $L_{\text {inv }}$ of the box can be computed in the limit of small $L_{\text {inv }}$. This calculation is given in [202]. In particular, in any even dimension $d$ one finds a universal term in the entropy that takes the form (up to numerical factor) $S \sim r_{+}^{d-2} h_{(d) \theta \theta}\left(r_{+}\right) L_{\mathrm{inv}}^{2} \ln L_{\mathrm{inv}}$ and is proportional to the value of the "obstruction tensor" on the black hole horizon. The direct calculation of such terms in the entanglement entropy on the CFT side is not yet available. 


\section{Can Entanglement Entropy Explain the Bekenstein-Haw- king Entropy of Black Holes?}

Entanglement entropy of a black hole is naturally proportional to the area of the black hole horizon. This property makes it very similar to the Bekenstein-Hawking entropy assigned to the horizon. This apparent similarity between the two entropies is the main motivation to raise the question of whether the Bekenstein-Hawking entropy is in fact entirely the entropy of entanglement. In this section we discuss problems, which this interpretation has to face, different approaches to solve them and difficulties, which still remain unsolved.

\subsection{Problems of interpretation of the Bekenstein-Hawking entropy as entanglement entropy}

Any approach, which wants to treat the Bekenstein-Hawking entropy as an entanglement entropy, has to answer the following questions:

(i) The entanglement entropy is a UV divergent quantity, while the Bekenstein-Hawking entropy is a finite quantity, defined with respect to Newton's constant, which has been measured in experiments. How can these two quantities be equal?

(ii) The entanglement entropy is proportional to the number of different field species, which exist in nature. On the other hand, the Bekenstein-Hawking entropy does not seem to depend on any number of fields. This problem is known as the "species puzzle".

(iii) We have seen that entanglement entropy due to fields, which are non-minimally coupled to gravity, the gauge bosons and gravitons, behave differently from the entropy due to minimallycoupled fields. Since the gauge bosons and gravitons are fields, which are clearly present in nature and thus should contribute to the entropy, does this contribution spoil the possibility of interpreting the black hole entropy as an entanglement entropy?

\subsection{Entanglement entropy in induced gravity}

One, possibly very natural, way, originally proposed by Jacobson [141], to attack these problems is to consider gravity as an induced phenomenon, in the spirit of Sakharov's ideas [190] (for a review on a modern touch on these ideas see [220]). In this approach the gravitational field is not fundamental but arises as a mean field approximation of the underlying quantum field theory of fundamental particles (the constituents). This is based on the fact that, even if there is no gravitational action at tree level, it will appear at one-loop. The details of this mechanism will, of course, depend on the concrete model.

Model with minimally-coupled fields. To start with, let us consider a simple model in which the constituents are minimally-coupled fields: we consider $N_{0}$ scalars and $N_{1 / 2}$ Dirac fermions. The induced gravitational action in this model, to lowest order in curvature, is

$$
W_{\text {ind }}=-\frac{1}{16 \pi G_{\text {ind }}} \int_{E} R \sqrt{g} d^{4} x
$$

where the induced Newton's constant is

$$
\frac{1}{G_{\text {ind }}}=\frac{N}{12 \pi \epsilon^{2}}, \quad N=N_{0}+2 N_{1 / 2},
$$

Living Reviews in Relativity

http: //www . livingreviews . org/lrr-2011-8 
$N$ being the number of field species in this model. The renormalization statement, which is valid for the minimally-coupled fields, guarantees that there is a precise balance between the induced Newton's constant and the entanglement entropy, so that

$$
S_{\mathrm{ent}}=\frac{N}{48 \pi \epsilon^{2}} A(\Sigma)=\frac{1}{4 G_{\mathrm{ind}}} A(\Sigma)=S_{\mathrm{BH}}
$$

i.e., the entanglement entropy of the constituents is precisely equal to the Bekenstein-Hawking entropy, expressed in terms of the induced Newton's constant (319). Thus, if at a fundamental level the constituents in nature were only minimal fields, the Bekenstein-Hawking entropy, as this example shows, would be explained as the entropy of entanglement. Of course, this example ignores the fact that there are elementary particles, namely the gauge bosons, which are non-minimally coupled.

Models with non-minimally coupled fields. In the model with minimal fields the induced Newton's constant (319) is set by the UV cutoff $\epsilon$. If one wants to deal with the UV finite quantities, one has to add fields, which contribute negatively to Newton's constant. Excluding non-physical fields with wrong statistics, the only possibility is to include non-minimally-coupled fields, scalars or vectors. Models of this type have been considered by Frolov, Fursaev and Zelnikov [95, 97, 87]. One considers a multiplet of scalar fields of mass $m_{s}$ and non-minimal coupling $\xi_{s}$ and a set of massive Dirac fields with mass $m_{d}$. The number of fields and their parameters are fine tuned so that the ultra-violet divergences in the cosmological constant and in Newton's constant are canceled. The induced Newton's constant then

$$
\frac{1}{G_{\mathrm{ind}}}=\frac{1}{12 \pi}\left(\sum_{s}\left(1-6 \xi_{s}\right) m_{s}^{2} \ln m_{s}^{2}+2 \sum_{d} m^{2} \ln m_{d}^{2}\right)
$$

is dominated by the mass of the heaviest constituents. However, as soon as we include the nonminimally-coupled fields the precise balance between Newton's constant and the entanglement entropy is violated, so that the Bekenstein-Hawking entropy $S_{\mathrm{BH}}=A(\Sigma) / 4 G_{\text {ind }}$, defined with respect to the induced Newton's constant, is no longer equal to the entanglement entropy. In the model considered in [95, 97, 87] (various models of a similar nature are considered in [88, 96, 93, $91,92,89,106,105])$, the exact relation between two entropies is

$$
S_{\mathrm{BH}}=S_{\mathrm{ent}}-Q,
$$

where the quantity $Q$ is determined by the expectation value of the non-minimally-coupled scalar fields on the horizon $\Sigma$

$$
Q=2 \pi \sum_{s} \xi_{s} \int_{\Sigma}<\phi_{s}^{2}>
$$

This quantity is UV divergent. For a single field it is similar to the quantity (142). Thus, the sharp difference between the entanglement entropy and the Bekenstein-Hawking entropy in this model can be summarized as follows: even though the induced Newton's constant is made UV finite, the entanglement entropy still (and, in fact, always) remains UV divergent. Thus, we conclude that, in the model of Frolov, Fursaev and Zelnikov, the entanglement entropy is clearly different from the Bekenstein-Hawking entropy.

\subsection{Entropy in brane-world scenario}

An interesting example where the Bekenstein-Hawking entropy is apparently induced in the correct way is given in [130]. This example is closely related to the AdS/CFT correspondence discussed 
in Section 7. In the Randall-Sundrum set-up [187] one may consider the regularized boundary, which appeared in our discussion of Section 7, as a 3-brane with $Z_{2}$ symmetry in an anti-de Sitter spacetime. In the framework of the AdS/CFT correspondence this brane has a description in terms of CFT coupled to gravity at a UV cutoff [124]. If the brane is placed at the distance $\rho=\epsilon^{2}$ from the anti-de Sitter boundary, one obtains that there is a dynamic gravity induced on the brane, with the induced Newton's constant

$$
1 / G_{N}=2 N^{2} /\left(\pi \epsilon^{2}\right),
$$

where $N$ is the number of colors in the superconformal $S U(N)$ Yang-Mills theory. $N^{2}$ in this case plays the role of the number of species. We notice that, according to the AdS/CFT dictionary, the parameter $\epsilon$, which is an infrared cut-off on the anti-de Sitter side, is, in fact, a UV cut-off on the CFT side. Now consider a black hole on the 3-brane. The Bekenstein-Hawking entropy can then be represented as follows

$$
S_{\mathrm{BH}}=\frac{A(\Sigma)}{4 G_{N}}=\frac{N^{2} A(\Sigma)}{2 \pi \epsilon^{2}}=S_{\mathrm{ent}}
$$

As Hawking, Maldacena and Strominger [130] suggested, the right hand side of Eq. (325) can be interpreted as the entanglement entropy of $N^{2}$ fields. This interpretation turns out to be the right one, if one uses the holographic entanglement entropy discussed in Section 7. Indeed, taking the leading divergent term in Eq. (317) and noting that, in a $Z_{2}$ brane configuration, this result should be multiplied by a factor of 2, we get exactly the right-hand side of Eq. (325). In [130] one considers de Sitter spacetime (so that Eq. (325) is the entropy of the de Sitter horizon in this case) on the brane since it is the simplest brane configuration one can construct in an anti-de Sitter spacetime. In [83] this proposal was extended to the holographic entanglement entropy of a black hole on a 2-brane solution found in [84]. The two-dimensional black hole is considered in [107]. Entropy of a generic black hole on the 3-brane was considered in [202].

However, there are certain open questions regarding this example. First of all, we should note that the weakly coupled $\mathcal{N}=4 S U(N)$ supermultiplet contains the Yang-Mills fields (gauge bosons), conformally-coupled scalars and the Weyl fermions [167]. Thus, it is a bit of a mystery how the entanglement entropy of these, mostly non-minimally coupled, fields (gauge bosons and scalars) has managed to become equal to the Bekenstein-Hawking entropy, when recalling the problems with the non-minimal coupling we have discussed in Section 3.16. A part of this mystery is the fact that the holographic regularization (which corresponds to infrared cut-off on the antide Sitter side) does not have a clear analog on the boundary side. Indeed, if we take, for example, a standard heat kernel regularization, we find that the term linear in the scalar curvature $R$ does not appear at all in the effective action produced by the weakly coupled $\mathcal{N}=4$ superconformal gauge multiplet.

\subsection{Gravity cut-off}

If we compare the two examples when the Bekenstein-Hawking entropy is correctly reproduced, the model of induced gravity with minimally-coupled constituents and the brane world model, we find that the success of the two models is strongly based on a precise relation between Newton's constant, the number of species and the UV cut-off. This relation can be reformulated in terms of the Planck mass $M_{\mathrm{PL}}\left(1 / G_{N} \sim M_{\mathrm{PL}}^{2}\right)$ and energy cutoff $\Lambda \sim 1 / \epsilon$,

$$
\Lambda=M_{\mathrm{PL}} / \sqrt{N}
$$

where the precise numerical pre-factor depends on the concrete model. It is amazing to note that exactly this relation (326) was proposed by Dvali $[77,79,78]$ to hold in general in a theory of

Living Reviews in Relativity

http: //www . livingreviews . org/lrr-2011-8 
quantum gravity coupled to a large number of matter species. The arguments, which were used to get this relation, although they include some thought experiments with black holes, are, in fact, unrelated to (and are thus independent of) the entropy. However, it is clear that relation (326), provided it is correct, helps to reproduce precisely the Bekenstein-Hawking entropy as an entropy of entanglement and automatically solves the species puzzle [80].

\subsection{Kaluza-Klein example}

One example of when relation (326) holds is the Kaluza-Klein model. In this model one starts with a $(4+n)$-dimensional theory of gravity, which is then compactified so that one has $n$ compact directions, forming, for example, an $n$-torus with characteristic size $R$, and 4 non-compact directions, which form our 4-dimensional geometry. The higher-dimensional Planck scale $\Lambda$ is considered fundamental in this model and plays the role of the UV cutoff, while the 4-dimensional Planck scale $M_{\mathrm{PL}}$ (or 4-dimensional Newton's constant) is derived,

$$
M_{\mathrm{PL}}^{2}=\Lambda^{2}(R \Lambda)^{n} \text {. }
$$

Suppose that, in higher dimensions, there are only one particle - the massless graviton. From the four-dimensional point of view one has, additionally to a single massless graviton, a theory of the tower of spin-2 massive Kaluza-Klein (KK) particles. Truncating the tower at the cut-off $\Lambda$, one finds that $N=(R \Lambda)^{n}$ is precisely the numebr of these Kaluza-Klein species. Thus, as was noted in $[77,79]$, the relation (327) is a particular example of relation (326) in which $N$ should be understood as the number of the KK species.

In the KK example, the entanglement entropy is equal to the Bekenstein-Hawking entropy as demonstrated by Dvali and Solodukhin [80]. Now consider a large black hole with horizon size $r_{g} \gg R$. Such a large black hole fills all compact directions so that, from the higher-dimensional point of view, the black hole horizon is a product of a 2 -sphere of radius $r_{g}$ and an $n$-dimensional torus of size $R$. The Bekenstein-Hawking entropy in the $(4+n)$-dimensional theory is

$$
S_{\mathrm{BH}}^{(4+n)}=4 \pi \Lambda^{n+2} r_{g}^{2} R^{n},
$$

where $4 \pi r_{g}^{2} R^{n}$ is the area of $(4+n)$-dimensional horizon. From the 4 -dimensional point of view this horizon is a 2-sphere of radius $r_{g}$ and the Bekenstein-Hawking entropy in the 4-dimensional theory is

$$
S_{\mathrm{BH}}^{(4)}=4 \pi M_{\mathrm{PL}}^{2} r_{g}^{2} .
$$

We observe that these two entropies are equal so that the two pictures, the higher dimensional and 4-dimensional one, are consistent. Let us now discuss the entanglement entropy. In the $(4+n)$-dimensional theory, there is only one field, the massless graviton. Its entropy is

$$
S_{\mathrm{ent}}^{(4+n)}=4 \pi r_{g}^{2} R^{n} \Lambda^{n+2},
$$

where the cut-off is the higher dimensional Planck scale $\Lambda$. On the other hand, in the 4-dimensional theory one computes the entanglement entropy of $N$ KK fields

$$
S_{\text {ent }}^{(4)}=N\left(4 \pi r_{g}^{2} \Lambda^{2}\right)=4 \pi r_{g}^{2} \Lambda^{2}(R \Lambda)^{n} .
$$

These two entropies are equal to each other so that the two ways to compute the entanglement entropy agree. Moreover, the entanglement entropy (331) and (330) exactly reproduces the Bekenstein-Hawking entropy (328) and (329).

However, discussing this result, we should note that the massless and massive gravitons are nonminimally coupled particles. It remains to be understood how the problem of the non-minimal coupling is overcome in this example. 


\section{Other Directions of Research}

In this section we briefly mention some other interesting directions of on-going research.

\subsection{Entanglement entropy in string theory}

It is generally believed that the problem of the entanglement entropy of a black hole can and should be resolved in string theory. This was originally suggested by Susskind and Uglum [213]. Indeed, taking that string theory is UV finite, the corresponding entropy calculation should result in a finite quantity. More specifically, the effective action of a closed string can be decomposed into powers of string coupling $g$ as $g^{2(n-1)}$, where $n$ is the genus of the string-world sheet. The string configurations with spherical topology, $n=0$, give a $1 / g^{2}$ contribution. In a low energy approximation this is exactly the contribution to Newton's constant $G \sim g^{2}$. Thus, one may expect that taking into account just $n=0$ closed string configurations, one will correctly reproduce both the entanglement entropy and Newton's constant. In the Euclidean formulation, the prescription of [213] is to look at the zero genus string world sheet, which intersects the Killing horizon. In the Lorentzian picture this corresponds to an open string with both ends attached to the horizon. The higher genus configurations should give some corrections to the $n=0$ result. This is a very attractive idea. However, a very little progress has been made in the literature to actually calculate the entanglement entropy directly in string theory. The reason is of course the technical complexity of the problem. Some support to the idea of Susskind and Uglum was found in the work of Kabat, Shenker and Strassler [151], where the entropy in a two-dimensional $O(N)$ invariant $\sigma$-model was considered. In particular, it was found that the state counting of the entropy in the UV regime may be lost if considered in the low energy (IR) regime. This type of behavior models the situation with the classical Bekenstein-Hawking entropy. Presumably this analysis could be generalized to the string theory $\sigma$-model considered either in optical target metric $[9,10]$ or in the Euclidean metric with a conical singularity at the horizon (as suggested in [33]). Possibly in the latter case the results obtained for strings on orbifolds [63] can be useful (see [50, 49, 51] for earlier works in this direction).

Another promising approach to attack the problem is to use some indirect methods based on dualities. For example, the AdS/CFT correspondence has been used in [25] to relate the entanglement entropy of a string propagating on a gravitational AdS background with a Killing horizon to the thermal entropy of field theory defined on a boundary of AdS and then, eventually, the thermal entropy to the Bekenstein-Hawking entropy of the horizon.

An interesting approach to the entanglement entropy of extremal black holes via $\mathrm{AdS}_{2} / \mathrm{CFT}_{1}$ duality is considered in [4, 192], where, in particular, one can identify the entanglement entropy and the microcanonical statistical entropy. This approach is based on the earlier work of Maldacena [168] in which the Hartle-Hawking state is identified with an entangled state of two copies of CFT, defined on two boundaries of the maximally-extended BTZ spacetime. In the accurately taken zero temperature limit, the reduced density matrix, obtained by tracing over the states of one copy of CFT, of the extremal black hole is shown to take the form

$$
\rho=\frac{1}{d(N)} \sum_{k=1}^{d(N)}|k><k|
$$

which describes the maximally-entangled state in the two copies of the $\mathrm{CFT}_{1}$ living on the two boundaries of global $\mathrm{AdS}_{2} . d(N)$ is the dimension of the Hilbert space of $\mathrm{CFT}_{1}$. The corresponding entanglement entropy $S=-\operatorname{Tr} \rho \ln \rho=\ln d(N)$ then is precisely equal to the micro-canonical entropy in the familiar counting of BPS states and thus is equal to the black-hole entropy [32].

Living Reviews in Relativity

http://www. livingreviews.org//rr-2011-8 


\subsection{Entanglement entropy in loop quantum gravity}

Another approach to quantum gravity, sometimes considered as competing with string theory, is loop quantum gravity. In this theory one considers polymeric excitations of the gravitational field represented by the states of spin networks. A spin network is a graph, a network of points with links representing the relation between points. Each link is labeled by a half-integer $j$ (the label stands for $S U(2)$ representations). To points, or vertices, of a spin network are attached a $S U(2)$ intertwiner, a $S U(2)$ invariant tensor between the representations attached to all the edges linked to the considered vertex. A simpler and more familiar object in particle physics is the Wilson loop. A surface $\Sigma$ is represented by vertices (punctures), which divide the spin network into two parts. By tracing over states of just one part of the network, one obtains a density matrix. The entanglement entropy then reduces to a sum over intersections of the spin network with the surface $\Sigma[56,165,66]$

$$
S(\Sigma)=\sum_{p=1}^{P} \ln \left(2 j_{p}+1\right),
$$

where $P$ is the number of punctures representing $\Sigma$. This quantity should be compared to eigenvalues of the operator of area,

$$
A(\Sigma)=8 \pi G \gamma \sum_{p=1}^{P} \sqrt{j_{p}\left(j_{p}+1\right)} .
$$

Both quantities scale as $P$ for large $P$, which indicates that the area law is correctly reproduced. However, the exact relation between the two quantities and the classical entropy $S_{\mathrm{BH}}=A(\Sigma) / G$ is not obvious due to ambiguities present in the formalism. The Immirzi parameter $\gamma$ is one of them. The question, whether the Bekenstein-Hawking entropy is correctly reproduced in this approach, is eventually related to the continuum limit of the theory [143]. As discussed by Jacobson [143], answering this question may require a certain renormalization of Newton's constant as well as area renormalization. Indeed, quantity (334) represents a microscopic area, which may be related to the macroscopic quantity in a non-trivial way. These issues remain open.

\subsection{Entropy in non-commutative theories and in models with minimal length}

One might have hoped that the UV divergence of the entanglement entropy could be cured in a natural way were the structure of spacetime modified on some fundamental level. For example, if spacetime becomes non-commutative at short distances. This idea was tested in the case of simple fuzzy spaces in $[68,67]$. Although the area law has been verified, the entanglement entropy appears to be sensitive to the size of the ignored region, a phenomenon, which may be understood as a UV-IR mixing typical for the non-commutative models.

A holographic calculation of the entanglement entropy in non-commutative Yang-Mills theory was considered in $[13,14]$. This calculation for a strip of width $l$ shows that for large values of $l \gg l_{c}$ compared to some characteristic length $l_{c} \sim \theta \lambda^{1 / 2} / \epsilon$, where $\theta$ is the parameter of noncommutativity and $\lambda=g_{Y M}^{2} N$ is the 't Hooft coupling, then the short-distance contribution to the entanglement entropy shows an area law of the form

$$
S \sim N_{\mathrm{eff}} \frac{A(\Sigma)}{\epsilon^{2}}, \quad N_{\mathrm{eff}}=N^{2}\left(\frac{\theta \lambda^{1 / 2}}{\epsilon}\right),
$$

while for smaller values $l \sim l_{c}$ the entropy is proportional to the volume. As seen from Eq. (335) the non-commutativity does not improve the UV behavior of the entropy but leads to the renor- 
malization of the effective number of degrees of freedom that may be interpreted as a manifestation of non-locality of the model.

The other related idea is to consider models in which the Heisenberg uncertainty relation is modified as $\Delta x \Delta p \geq \frac{\hbar}{2}\left(1+\lambda^{2}(\Delta p)^{2}\right)$, which shows that there exists a minimal length $\Delta x \geq \hbar \lambda$ (for a review on the models of this type see [113]). In a brick-wall calculation the presence of this minimal length will regularize the entropy as discussed in [27, 225, 210, 154, 156].

\subsection{Transplanckian physics and entanglement entropy}

One way to check whether the entanglement entropy is sensitive to the way the conventional theory is completed in the UV regime is to study the possible modifications of the standard Lorentz invariant dispersion relation $\omega^{2}=\mathbf{k}^{2}$ at large values of momentum $\mathbf{k}$ (or at short distances). A typical modification is to break the Lorentz invariance as follows $\omega^{2}=\mathbf{k}^{2}+f\left(\mathbf{k}^{2}\right)$. This issue was studied in [147] and [40] in the context of the brick-wall model. However, the conclusions made in these papers are opposite. According to [147] the entropy is still UV divergent, although the degree of divergence is modified in a way, which depends on the form of function $f\left(\mathbf{k}^{2}\right)$. On the other hand, [40] claims that the entropy can be made completely UV finite. In a similar claim [186] suggests that the short-distance finiteness of the 2-point correlation function should imply the UV finiteness of the entanglement entropy. The entanglement entropy in a wide class of theories characterized by modified (Lorentz invariant or not) field operators (so that the UV behavior of the modified propagator is improved compared to the standard one) was calculated in [184]. The conclusion reached in [184] (see also discussion in Sections 2.12 and 3.13 of this review) agrees with that of [147]: no matter how good the UV behavior of the propagator is, the entanglement entropy remains UV divergent. That the short-distance regularity of correlation functions does not necessarily imply that the entanglement entropy is UV finite was pointed out in [183].

\subsection{Entropy of more general states}

The pure quantum state, which is the starting point in the entanglement-entropy calculation, should not necessarily be a vacuum state. According to [55, 53, 52, 54], if one starts with a mixed state of the vacuum and an excited state, the entanglement entropy receives some power law corrections,

$$
S \sim \frac{A}{\epsilon^{2}}\left(1+c\left(\frac{A}{\epsilon^{2}}\right)^{-\beta}\right),
$$

with $\beta$ always less than unity, and the power law correction is due to the excited state.

\subsection{Non-unitary time evolution}

An interesting issue discussed in the literature is the time evolution of the entanglement entropy. It was suggested in [26] that the eigenvalues of a reduced density matrix depend on time $t$. This is not possible if the time evolution of the density matrix is described by a unitary operator. Thus, the time evolution should be nonunitary. In particular, the entanglement entropy should depend on time $t$. Similar conclusions have been made in [30] and $[158,159,160]$, where, in particular, it was shown that the entanglement entropy is an increasing function of time. These observations may have interesting applications for black holes. As was proposed by Hawking [129] the evolution in time of a black hole should be nonunitary, so that a pure initial state may evolve into a mixed state. From the entanglement point of view, this behavior appears not to be in contradiction with the principles of quantum mechanics, rather it is a simple consequence of the entangled nature of the system. The irreversible loss of information due to entanglement is also seen from the evolution of the entropy under the Renorm-Group (RG) flow [203, 164].

Living Reviews in Relativity

http: //www . livingreviews . org/lrr-2011-8 


\section{Concluding remarks}

Since the inspiring work of Srednicki in 1993 we have come a long way in understanding the entanglement entropy. Of course, the main motivation for this research always was and still is the attempt to consider the Bekenstein-Hawking entropy as an entropy of entanglement. If successful, this approach would give a universal explication for the entropy of black holes, valid for black holes of any size and mass and carrying any charges. Unfortunately, we are not yet at that point. Many important unresolved problems remain. However, after 17 years of continuous progress it would not be too surprising if we were actually not that far from the final answer. Speaking of future developments, I think that further progress could be made in two main directions: understanding entanglement entropy directly in string theory and resolving the puzzle of non-minimal coupling. Hopefully, at some not-so-distant point in time, the efforts made in these directions will provide us with the still missing elements in the picture.

\section{Acknowledgments}

I would like to thank my collaborators and friends A. Barvinsky, G. Dvali, G. 't Hooft, W. Israel, V. Frolov, D. Fursaev, R. Mann, R. Myers, S. Mukhanov, D. Nesterov and A. Zelnikov for fruitful collaboration and many inspiring discussions. I thank G. 't Hooft, R. Myers and R. Mann for reading this review and giving valuable remarks. I am indebted to my colleagues N. Mohammedi, L. Villain and, especially, to S. Nicolis for reading this review and their many comments, which helped to improve the presentation. As always, the writing of this review would not be possible without the constant support of my family. 


\section{References}

[1] Aharony, O., Gubser, S.S., Maldacena, J.M., Ooguri, H. and Oz, Y., "Large $N$ field theories, string theory and gravity", Phys. Rep., 323, 183-386, (2000). [DOI], [arXiv:hep-th/9905111 [hep-th]]. (Cited on page 68.)

[2] Allen, B., "Does statistical mechanics equal one loop field theory?", Phys. Rev. D, 33, 3640, (1986). [DOI]. (Cited on pages 41 and 46.)

[3] Allen, B. and Ottewill, A.C., "Effects of curvature couplings for quantum fields on cosmic string space-times", Phys. Rev. D, 42, 2669-2677, (1990). [DOI]. (Cited on page 24.)

[4] Azeyanagi, T., Nishioka, T. and Takayanagi, T., "Near extremal black hole entropy as entanglement entropy via $\mathrm{AdS}_{2} / \mathrm{CFT}_{1}$ ", Phys. Rev. D, 77, 064005, (2008). [DOI], [arXiv:0710.2956 [hep-th]]. (Cited on pages 24 and 78.)

[5] Bañados, M., Henneaux, M., Teitelboim, C. and Zanelli, J., "Geometry of the (2+1) black hole", Phys. Rev. D, 48, 1506-1525, (1993). [DOI], [arXiv:gr-qc/9302012 [gr-qc]]. (Cited on page 52.)

[6] Bañados, M., Teitelboim, C. and Zanelli, J., "The black hole in three-dimensional spacetime", Phys. Rev. Lett., 69, 1849-1851, (1992). [DOI], [arXiv:hep-th/9204099 [hep-th]]. (Cited on page 52.)

[7] Banados, M., Teitelboim, C. and Zanelli, J., "Black hole entropy and the dimensional continuation of the Gauss-Bonnet theorem", Phys. Rev. Lett., 72, 957-960, (1994). [DOI], [arXiv:grqc/9309026 [gr-qc]]. (Cited on pages 21 and 23.)

[8] Banerjee, S., Gupta, R.K. and Sen, A., "Logarithmic Corrections to Extremal Black Hole Entropy from Quantum Entropy Function", J. High Energy Phys., 2011(03), 147, (2011). [DOI], [arXiv:1005.3044 [hep-th]]. (Cited on page 66.)

[9] Barbón, J.L.F., "Horizon divergences of fields and strings in black hole backgrounds", Phys. Rev. D, 50, 2712-2718, (1994). [DOI], [arXiv:hep-th/9402004]. (Cited on pages 44, 49, and 78.)

[10] Barbón, J.L.F., "Remarks on thermal strings outside black holes", Phys. Lett. B, 339, 41-48, (1994). [DOI], [arXiv:hep-th/9406209]. (Cited on pages 44, 49, and 78.)

[11] Barbón, J.L.F., "Holographic avatars of entanglement entropy", Nucl. Phys. B (Proc. Suppl.), 192-193, 12-26, (2009). [DOI]. (Cited on page 6.)

[12] Barbón, J.L.F. and Emparan, R., "On quantum black hole entropy and Newton constant renormalization", Phys. Rev. D, 52, 4527-4539, (1995). [DOI], [arXiv:hep-th/9502155]. (Cited on page 33.)

[13] Barbón, J.L.F. and Fuertes, C.A., "Holographic entanglement entropy probes (non)locality", J. High Energy Phys., 2008(04), 096, (2008). [DOI], [arXiv:0803.1928 [hep-th]]. (Cited on page 79.)

[14] Barbón, J.L.F. and Fuertes, C.A., "A Note on the extensivity of the holographic entanglement entropy", J. High Energy Phys., 2008(05), 053, (2008). [DOI], [arXiv:0801.2153 [hep-th]]. (Cited on page 79.)

[15] Barvinsky, A.O., Frolov, V.P. and Zelnikov, A., "Wave function of a black hole and the dynamical origin of entropy", Phys. Rev. D, 51, 1741-1763, (1995). [DOI], [arXiv:gr-qc/9404036]. (Cited on pages 19 and 49.) 
[16] Barvinsky, A.D. and Solodukhin, S.N., "Non-minimal coupling, boundary terms and renormalization of the Einstein-Hilbert action and black hole entropy", Nucl. Phys. B, 479, 305318, (1996). [DOI], [arXiv:gr-qc/9512047]. (Cited on page 37.)

[17] Bastianelli, F., Frolov, S. and Tseytlin, A.A., "Conformal anomaly of $(2,0)$ tensor multiplet in six dimensions and AdS/CFT correspondence", J. High Energy Phys., 2000(02), 013, (2000). [DOI], [arXiv:hep-th/0001041 [hep-th]]. (Cited on pages 64 and 65.)

[18] Bekenstein, J.D., "Black holes and the second law", Lett. Nuovo Cimento, 4, 737-740, (1972). [DOI]. (Cited on page 5.)

[19] Bekenstein, J.D., "Black holes and entropy", Phys. Rev. D, 7, 2333-2346, (1973). [DOI]. (Cited on page 5.)

[20] Bekenstein, J.D., "Generalized second law of thermodynamics in black-hole physics", Phys. Rev. D, 9, 3292-3300, (1974). [DOI]. (Cited on page 5.)

[21] Bekenstein, J.D., "Do We Understand Black Hole Entropy?", in Jantzen, R.T. and Mac Keiser, G., eds., The Seventh Marcel Grossmann Meeting on Recent Developments in Theoretical and Experimental General Relativity, Gravitation and Relativistic Field Theories, Proceedings of the meeting held at Stanford University, 24-30 July 1994, pp. 39-58, (World Scientific, Singapore; River Edge, NJ, 1994). [gr-qc/9409015]. (Cited on page 6.)

[22] Birrell, N.D. and Davies, P.C.W., Quantum fields in curved space, Cambridge Monographs on Mathematical Physics, (Cambridge University Press, Cambridge; New York, 1982). [Google Books]. (Cited on pages 31 and 62.)

[23] Bombelli, L., Koul, R.K., Lee, J. and Sorkin, R.D., "Quantum source of entropy for black holes", Phys. Rev. D, 34, 373-383, (1986). [DOI]. (Cited on page 5.)

[24] Bousso, R., "The holographic principle", Rev. Mod. Phys., 74, 825-874, (2002). [DOI], [arXiv:hep-th/0203101 [hep-th]]. (Cited on page 68.)

[25] Brustein, R., Einhorn, M.B. and Yarom, A., "Entanglement interpretation of black hole entropy in string theory", J. High Energy Phys., 2006(01), 098, (2006). [DOI], [arXiv:hepth/0508217 [hep-th]]. (Cited on page 78.)

[26] Brustein, R., Einhorn, M.B. and Yarom, A., "Entanglement and Nonunitary Evolution", J. High Energy Phys., 2007(04), 086, (2007). [DOI], [arXiv:hep-th/0609075 [hep-th]]. (Cited on page 80.)

[27] Brustein, R. and Kupferman, J., "Black hole entropy divergence and the uncertainty principle", Phys. Rev. D, 83, 124014, (2011). [DOI], [arXiv:1010.4157 [hep-th]]. (Cited on page 80.)

[28] Bytsenko, A.A., Vanzo, L. and Zerbini, S., "Quantum correction to the entropy of the $(2+1)$ dimensional black hole", Phys. Rev. D, 57, 4917-4924, (1998). [DOI], [arXiv:gr-qc/9710106 [gr-qc]]. (Cited on page 28.)

[29] Calabrese, P. and Cardy, J.L., "Entanglement entropy and quantum field theory", J. Stat. Mech., 2004(06), P06002, (2004). [DOI], [arXiv:hep-th/0405152]. (Cited on page 9.)

[30] Calabrese, P. and Cardy, J.L., "Evolution of entanglement entropy in one-dimensional systems", J. Stat. Mech., 2005(04), P04010, (2005). [DOI], [arXiv:cond-mat/0503393 [cond-mat]]. (Cited on page 80.) 
[31] Calabrese, P. and Cardy, J., "Entanglement entropy and conformal field theory", J. Phys. A: Math. Theor., 42, 504005, (2009). [DOI], [arXiv:0905.4013 [cond-mat.stat-mech]]. (Cited on page 6.)

[32] Callan, C.G. and Maldacena, J.M., "D-brane Approach to Black Hole Quantum Mechanics", Nucl. Phys. B, 472, 591-610, (1996). [DOI], [arXiv:hep-th/9602043]. (Cited on page 78.)

[33] Callan Jr, C.G. and Wilczek, F., "On geometric entropy", Phys. Lett. B, 333, 55-61, (1994). [DOI], [arXiv:hep-th/9401072]. (Cited on pages 5, 9, 25, 31, and 78.)

[34] Camporesi, R., "Harmonic analysis and propagators on homogeneous spaces", Phys. Rep., 196, 1-134, (1990). [DOI]. (Cited on page 57.)

[35] Carlip, S., "Logarithmic corrections to black hole entropy from the Cardy formula", Class. Quantum Grav., 17, 4175-4186, (2000). [DOI], [arXiv:gr-qc/0005017]. (Cited on page 66.)

[36] Carlson, F., Sur une classe de séries de Taylor, Ph.D. Thesis, (Uppsala University, Uppsala, 1914). (Cited on page 11.)

[37] Casini, H. and Huerta, M., "Entanglement entropy in free quantum field theory", J. Phys. A: Math. Theor., 42, 504007, (2009). [DOI], [arXiv:0905.2562 [hep-th]]. (Cited on page 6.)

[38] Casini, H. and Huerta, M., "Entanglement entropy for the n-sphere", Phys. Lett. B, 694, 167-171, (2010). [DOI], [arXiv:1007.1813 [hep-th]]. (Cited on page 59.)

[39] Casini, H., Huerta, M. and Myers, R.C., "Towards a derivation of holographic entanglement entropy", J. High Energy Phys., 2011(05), 036, (2011). [DOI], [arXiv:1102.0440 [hep-th]]. (Cited on page 65.)

[40] Chang, D., Chu, C.-S. and Lin, F.-L., "Transplanckian entanglement entropy", Phys. Lett. $B, \mathbf{5 8 3}, 192-198,(2004)$. [DOI], [arXiv:hep-th/0306055 [hep-th]]. (Cited on page 80.)

[41] Chang-Young, E., Lee, D. and Yoon, M., "Rotating Black Hole Entropy from Two Different Viewpoints", Class. Quantum Grav., 26, 155011, (2009). [DOI], [arXiv:0811.3294 [hep-th]]. (Cited on page 45.)

[42] Cheeger, J., "Spectral geometry of singular Riemannian spaces", J. Differ. Geom., 18(4), 575-657, (1983). (Cited on page 24.)

[43] Christensen, S.M. and Duff, M.J., "New Gravitational Index Theorems and Supertheorems", Nucl. Phys. B, 154, 301-342, (1979). [DOI]. (Cited on page 35.)

[44] Christensen, S.M. and Duff, M.J., "Quantizing Gravity with a Cosmological Constant", Nucl. Phys. B, 170, 480-506, (1980). [DOI]. (Cited on page 35.)

[45] Cognola, G., "Thermodynamics of scalar fields in Kerr's geometry", Phys. Rev. D, 57, 62926296, (1998). [DOI], [arXiv:gr-qc/9710118 [gr-qc]]. (Cited on pages 31 and 45.)

[46] Cognola, G., Kirsten, K. and Vanzo, L., "Free and self-interacting scalar fields in the presence of conical singularities", Phys. Rev. D, 49, 1029-1038, (1994). [DOI], [arXiv:hep-th/9308106 [hep-th]]. (Cited on pages 28 and 39.)

[47] Cognola, G., Vanzo, L. and Zerbini, S., "One loop quantum corrections to the entropy for a four-dimensional eternal black hole", Class. Quantum Grav., 12, 1927-1936, (1995). [DOI], [arXiv:hep-th/9502006 [hep-th]]. (Cited on page 28.) 
[48] Cognola, G., Vanzo, L. and Zerbini, S., "One loop quantum corrections to the entropy for an extremal Reissner-Nordström black hole", Phys. Rev. D, 52, 4548-4553, (1995). [DOI], [arXiv:hep-th/9504064 [hep-th]]. (Cited on page 28.)

[49] Dabholkar, A., "Quantum corrections to black hole entropy in string theory", Phys. Lett. B, 347, 222-229, (1995). [DOI], [arXiv:hep-th/9409158 [hep-th]]. (Cited on page 78.)

[50] Dabholkar, A., "Strings on a cone and black hole entropy", Nucl. Phys. B, 439, 650-664, (1995). [DOI], [arXiv:hep-th/9408098 [hep-th]]. (Cited on page 78.)

[51] Dabholkar, A., "Tachyon condensation and black hole entropy", Phys. Rev. Lett., 88, 091301, (2002). [DOI], [arXiv:hep-th/0111004 [hep-th]]. (Cited on page 78.)

[52] Das, S. and Shankaranarayanan, S., "How robust is the entanglement entropy-area relation?", Phys. Rev. D, 73, 121701, (2006). [DOI], [arXiv:gr-qc/0511066]. (Cited on page 80.)

[53] Das, S., Shankaranarayanan, S. and Sur, S., "Power-law corrections to entanglement entropy of black holes", Phys. Rev. D, 77, 064013, (2008). [DOI], [arXiv:0705.2070 [gr-qc]]. (Cited on page 80.)

[54] Das, S., Shankaranarayanan, S. and Sur, S., "Black hole entropy from entanglement: A review", in Everett, M. and Pedroza, L., eds., Horizons in World Physics, Horizons in World Physics, 268, (Nova Science, Hauppauge, NY, 2009). [arXiv:0806.0402 [gr-qc]]. (Cited on pages 6 and 80.)

[55] Das, S., Shankaranarayanan, S. and Sur, S., "Entanglement and corrections to BekensteinHawking entropy", arXiv, e-print, (2010). [arXiv:1002.1129 [gr-qc]]. (Cited on page 80.)

[56] Dasgupta, A., "Semi-classical quantisation of space-times with apparent horizons", Class. Quantum Grav., 23, 635-672, (2006). [DOI], [arXiv:gr-qc/0505017 [gr-qc]]. (Cited on page 79.)

[57] de Alwis, S.P. and Ohta, N., "On the entropy of quantum fields in black hole backgrounds", arXiv, e-print, (1994). [arXiv:hep-th/9412027 [hep-th]]. (Cited on page 49.)

[58] de Alwis, S.P. and Ohta, N., "Thermodynamics of quantum fields in black hole backgrounds", Phys. Rev. D, 52, 3529-3542, (1995). [DOI], [arXiv:hep-th/9504033 [hep-th]]. (Cited on page 49.)

[59] de Boer, J., Kulaxizi, M. and Parnachev, A., "Holographic Entanglement Entropy in Lovelock Gravities", arXiv, e-print, (2011). [arXiv:1101.5781 [hep-th]]. (Cited on page 16.)

[60] de Haro, S., Solodukhin, S.N. and Skenderis, K., "Holographic reconstruction of space-time and renormalization in the AdS/CFT correspondence", Commun. Math. Phys., 217, 595622, (2001). [DOI], [arXiv:hep-th/0002230 [hep-th]]. (Cited on pages 68, 69, and 71.)

[61] De Nardo, L., Fursaev, D.V. and Miele, G., "Heat-kernel coefficients and spectra of the vector Laplacians on spherical domains with conical singularities", Class. Quantum Grav., 14, 1059-1078, (1997). [DOI], [arXiv:hep-th/9610011]. (Cited on page 28.)

[62] Demers, J.-G., Lafrance, R. and Myers, R.C., "Black hole entropy without brick walls", Phys. Rev. D, 52, 2245-2253, (1995). [DOI], [arXiv:gr-qc/9503003]. (Cited on pages 33, 44, and 45.)

[63] Dixon, L.J., Harvey, J.A., Vafa, C. and Witten, E., "Strings on Orbifolds", Nucl. Phys. B, 261, 678-686, (1985). [DOI]. (Cited on page 78.) 
[64] Donnelly, H., "Spectrum and the fixed point sets of isometries. I", Math. Ann., 224, 161-170, (1976). [DOI]. (Cited on page 24.)

[65] Donnelly, H., "Asymptotic expansions for the compact quotients of properly discontinuous group actions", Illinois J. Math., 23, 485-496, (1979). Online version (accessed 20 October 2011):

http://projecteuclid.org/euclid.ijm/1256048110. (Cited on page 24.)

[66] Donnelly, W., "Entanglement entropy in loop quantum gravity", Phys. Rev. D, 77, 104006, (2008). [DOI], [arXiv:0802.0880 [gr-qc]]. (Cited on page 79.)

[67] Dou, D., "Comments on the Entanglement Entropy on Fuzzy Spaces", Mod. Phys. Lett. A, 24, 2467-2480, (2009). [DOI], [arXiv:0903.3731 [gr-qc]]. (Cited on page 79.)

[68] Dou, D. and Ydri, B., "Entanglement entropy on fuzzy spaces", Phys. Rev. D, 74, 044014, (2006). [DOI], [arXiv:gr-qc/0605003 [gr-qc]]. (Cited on page 79.)

[69] Dowker, J.S., "Quantum field theory on a cone", J. Phys. A: Math. Gen., 10, 115-124, (1977). [DOI]. (Cited on pages 11, 12, and 24.)

[70] Dowker, J.S., "Effective actions with fixed points", Phys. Rev. D, 50, 6369-6373, (1994). [DOI], [arXiv:hep-th/9406144 [hep-th]]. (Cited on pages 30 and 63.)

[71] Dowker, J.S., "Remarks on geometric entropy", Class. Quantum Grav., 11, L55-L60, (1994). [DOI], [arXiv:hep-th/9401159]. (Cited on page 25.)

[72] Dowker, J.S., "Hyperspherical entanglement entropy", J. Phys. A: Math. Theor., 43, 445402, (2010). [DOI], [arXiv:1007.3865 [hep-th]]. (Cited on page 59.)

[73] Dowker, J.S. and Kennedy, G., "Finite temperature and boundary effects in static spacetimes", J. Phys. A: Math. Gen., 11, 895-920, (1978). [DOI]. (Cited on page 48.)

[74] Dowker, J.S. and Schofield, J.P., "High temperature expansion of the free energy of a massive scalar field in a curved space", Phys. Rev. D, 38, 3327, (1988). [DOI]. (Cited on page 48.)

[75] Dowker, J.S. and Schofield, J.P., "Chemical potentials in curved space", Nucl. Phys. B, 327, 267, (1989). [DOI]. (Cited on page 48.)

[76] Duff, M.J., "Twenty years of the Weyl anomaly", Class. Quantum Grav., 11, 1387-1404, (1994). [DOI], [arXiv:hep-th/9308075]. (Cited on page 62.)

[77] Dvali, G., "Black holes and large $N$ species solution to the hierarchy problem", Fortschr. Phys., 58, 528-536, (2010). [DOI], [arXiv:0706.2050 [hep-th]]. (Cited on pages 76 and 77.)

[78] Dvali, G., "Nature of Microscopic Black Holes and Gravity in Theories with Particle Species", Int. J. Mod. Phys. A, 25, 602-615, (2010). [DOI], [arXiv:0806.3801 [hep-th]]. (Cited on page 76.)

[79] Dvali, G. and Redi, M., "Black Hole Bound on the Number of Species and Quantum Gravity at LHC", Phys. Rev. D, 77, 045027, (2008). [DOI], [arXiv:0710.4344 [hep-th]]. (Cited on pages 76 and 77.$)$

[80] Dvali, G. and Solodukhin, S.N., "Black Hole Entropy and Gravity Cutoff", arXiv, e-print, (2008). [arXiv:0806.3976 [hep-th]]. (Cited on page 77.) 
[81] Eisert, J., Cramer, M. and Plenio, M.B., "Colloquium: Area laws for the entanglement entropy", Rev. Mod. Phys., 82, 277-306, (2010). [DOI], [arXiv:0808.3773 [quant-ph]]. (Cited on page 6.)

[82] Emparan, R., "Heat kernels and thermodynamics in Rindler space", Phys. Rev. D, 51, 57165719, (1995). [DOI], [arXiv:hep-th/9407064 [hep-th]]. (Cited on page 28.)

[83] Emparan, R., "Black hole entropy as entanglement entropy: a holographic derivation", $J$. High Energy Phys., 06(2006), 012, (2006). [DOI], [arXiv:hep-th/0603081 [hep-th]]. (Cited on pages 69,70 , and 76.$)$

[84] Emparan, R., Horowitz, G.T. and Myers, R.C., "Exact description of black holes on branes", J. High Energy Phys., 2000(01), 007, (2000). [DOI], [arXiv:hep-th/9911043 [hep-th]]. (Cited on pages 69 and 76. )

[85] Fiola, T.M., Preskill, J., Strominger, A. and Trivedi, S.P., "Black hole thermodynamics and information loss in two-dimensions", Phys. Rev. D, 50, 3987-4014, (1994). [DOI], [arXiv:hepth/9403137 [hep-th]]. (Cited on page 25.)

[86] Frolov, V.P., "Why the entropy of a black hole is A/4?", Phys. Rev. Lett., 74, 3319-3322, (1995). [DOI], [arXiv:gr-qc/9406037]. (Cited on page 41.)

[87] Frolov, V.P. and Fursaev, D.V., "Mechanism of generation of black hole entropy in Sakharov's induced gravity", Phys. Rev. D, 56, 2212-2225, (1997). [DOI], [arXiv:hep-th/9703178]. (Cited on page 75. )

[88] Frolov, V.P. and Fursaev, D.V., "Plenty of nothing: Black hole entropy in induced gravity", arXiv, e-print, (1997). [arXiv:hep-th/9705207]. (Cited on page 75.)

[89] Frolov, V.P. and Fursaev, D., "Black hole entropy in induced gravity: Reduction to 2D quantum field theory on the horizon", Phys. Rev. D, 58, 124009, (1998). [DOI], [arXiv:hepth/9806078]. (Cited on pages 14 and 75.)

[90] Frolov, V.P. and Fursaev, D.V., "Thermal fields, entropy, and black holes", Class. Quantum Grav., 15, 2041-2074, (1998). [DOI], [arXiv:hep-th/9802010]. (Cited on page 6.)

[91] Frolov, V.P. and Fursaev, D.V., "Statistical mechanics of charged black holes in induced Einstein-Maxwell gravity", Phys. Rev. D, 61, 064010, (2000). [DOI], [arXiv:hep-th/9910006]. (Cited on page 75.)

[92] Frolov, V.P. and Fursaev, D.V., "Statistical mechanics on axially-symmetric space-times with the Killing horizon and entropy of rotating black holes in induced gravity", Phys. Rev. D, 61, 024007, (2000). [DOI], [arXiv:gr-qc/9907046]. (Cited on pages 31, 45, and 75.)

[93] Frolov, V.P., Fursaev, D., Gegenberg, J. and Kunstatter, G., "Thermodynamics and statistical mechanics of induced Liouville gravity", Phys. Rev. D, 60, 024016, (1999). [DOI], [arXiv:hep-th/9901087]. (Cited on page 75.)

[94] Frolov, V.P., Fursaev, D.V. and Zelnikov, A., "Black Hole Entropy: Off-Shell vs On-Shell", Phys. Rev. D, 54, 2711-2731, (1996). [DOI], [arXiv:hep-th/9512184]. (Cited on page 28.)

[95] Frolov, V.P., Fursaev, D.V. and Zelnikov, A., "Black Hole Entropy: Thermodynamics, Statistical-Mechanics and Subtraction Procedure", Phys. Lett. B, 382, 220-226, (1996). [DOI], [arXiv:hep-th/9603175]. (Cited on page 75.) 
[96] Frolov, V.P., Fursaev, D.V. and Zelnikov, A., "Black hole statistical mechanics and induced gravity", Nucl. Phys. B (Proc. Suppl.), 57, 192-196, (1997). [DOI]. (Cited on page 75.)

[97] Frolov, V.P., Fursaev, D.V. and Zelnikov, A., "Statistical origin of black hole entropy in induced gravity", Nucl. Phys. B, 486, 339-352, (1997). [DOI], [arXiv:hep-th/9607104]. (Cited on pages 6 and 75 .)

[98] Frolov, V.P., Israel, W. and Solodukhin, S.N., "On One-loop Quantum Corrections to the Thermodynamics of Charged Black Holes", Phys. Rev. D, 54, 2732-2745, (1996). [DOI], [arXiv:hep-th/9602105]. (Cited on page 51.)

[99] Frolov, V.P. and Novikov, I., "Dynamical origin of the entropy of a black hole", Phys. Rev. D, 48, 4545-4551, (1993). [DOI], [arXiv:gr-qc/9309001]. (Cited on page 5.)

[100] Frolov, V.P. and Serebryanyi, E.M., "Vacuum polarization in the gravitational field of a cosmic string", Phys. Rev. D, 35, 3779-3782, (1987). [DOI]. (Cited on page 24.)

[101] Fursaev, D.V., "Spectral geometry and one loop divergences on manifolds with conical singularities", Phys. Lett. B, 334, 53-60, (1994). [DOI], [arXiv:hep-th/9405143]. (Cited on pages 11, 12, 24, and 62.)

[102] Fursaev, D.V., "Black hole thermodynamics and renormalization", Mod. Phys. Lett. A, 10, 649-656, (1995). [DOI], [arXiv:hep-th/9408066]. (Cited on page 28.)

[103] Fursaev, D.V., "Temperature and entropy of a quantum black hole and conformal anomaly", Phys. Rev. D, 51, 5352-5355, (1995). [DOI], [arXiv:hep-th/9412161]. (Cited on pages 63 and 66.)

[104] Fursaev, D.V., "Euclidean and canonical formulations of statistical mechanics in the presence of Killing horizons", Nucl. Phys. B, 524, 447-468, (1998). [DOI], [arXiv:hep-th/9709213]. (Cited on pages 28, 41, 45, and 49.)

[105] Fursaev, D.V., "Energy, Hamiltonian, Noether charge, and black holes", Phys. Rev. D, 59, 064020, (1999). [DOI], [arXiv:hep-th/9809049]. (Cited on page 75.)

[106] Fursaev, D.V., "Black hole entropy in induced gravity and information loss", Nucl. Phys. B (Proc. Suppl.), 88, 277-280, (2000). [DOI], [arXiv:gr-qc/9911065]. (Cited on page 75.)

[107] Fursaev, D.V., "Black Hole Thermodynamics, Induced Gravity and Gravity in Brane Worlds", arXiv, e-print, (2000). [arXiv:hep-th/0009164]. (Cited on page 76.)

[108] Fursaev, D.V., "Entanglement entropy in critical phenomena and analogue models of quantum gravity", Phys. Rev. D, 73, 124025, (2006). [DOI], [arXiv:hep-th/0602134]. (Cited on page 16.)

[109] Fursaev, D.V., "Proof of the holographic formula for entanglement entropy", J. High Energy Phys., 2006(09), 018, (2006). [DOI], [arXiv:hep-th/0606184]. (Cited on page 68.)

[110] Fursaev, D.V. and Miele, G., "Cones, Spins and Heat Kernels", Nucl. Phys. B, 484, 697-723, (1997). [DOI], [arXiv:hep-th/9605153]. (Cited on pages 28 and 35.)

[111] Fursaev, D.V. and Solodukhin, S.N., "On the description of the Riemannian geometry in the presence of conical defects", Phys. Rev. D, 52, 2133-2143, (1995). [DOI], [arXiv:hepth/9501127]. (Cited on pages 5, 21, 22, 23, and 62.) 
[112] Fursaev, D.V. and Solodukhin, S.N., "On one-loop renormalization of black hole entropy", Phys. Lett. B, 365, 51-55, (1996). [DOI], [arXiv:hep-th/9412020]. (Cited on pages 33, 50, and 62.)

[113] Garay, L.J., "Quantum gravity and minimum length", Int. J. Mod. Phys. A, 10, 145-166, (1995). [DOI], [arXiv:gr-qc/9403008 [gr-qc]]. (Cited on page 80.)

[114] Ghosh, A. and Mitra, P., "Entropy in dilatonic black hole background", Phys. Rev. Lett., 73, 2521-2523, (1994). [DOI], [arXiv:hep-th/9406210 [hep-th]]. (Cited on page 28.)

[115] Ghosh, A. and Mitra, P., "Entropy for extremal Reissner-Nordstrom black holes", Phys. Lett. B, 357, 295-299, (1995). [DOI], [arXiv:hep-th/9411128 [hep-th]]. (Cited on page 28.)

[116] Ghosh, A. and Mitra, P., "Quantum correction to the thermodynamical entropy of a black hole", Phys. Rev. D, 56, 3568-3570, (1997). [DOI], [arXiv:gr-qc/9706054 [gr-qc]]. Erratum: 10.1103/PhysRevD.57.1316. (Cited on page 28.)

[117] Ghosh, K., "Entropy of scalar fields in (3+1)-dimensional constant curvature black hole background", Phys. Rev. D, 60, 104003, (1999). [DOI], [arXiv:gr-qc/9809063 [gr-qc]]. (Cited on page 28.)

[118] Ghosh, K., "Entropy of scalar field in (3+1)-dimensional Reissner-Nordstrom de Sitter black hole background", arXiv, e-print, (2002). [arXiv:gr-qc/0212060 [gr-qc]]. (Cited on page 28.)

[119] Gibbons, G.W. and Hawking, S.W., "Action Integrals and Partition Functions in Quantum Gravity", Phys. Rev. D, 15, 2752-2756, (1977). [DOI]. (Cited on page 40.)

[120] Gibbons, G.W. and Maeda, K., "Black holes and membranes in higher-dimensional theories with dilaton fields", Nucl. Phys. B, 298, 741-775, (1988). [DOI]. (Cited on page 27.)

[121] Gibbons, G.W. and Perry, M.J., "Black holes and thermal Green functions", Proc. R. Soc. London, Ser. A, 358, 467-494, (1978). (Cited on page 20.)

[122] Graham, C.R. and Hirachi, V., "The Ambient Obstruction Tensor and Q-Curvature", arXiv, e-print, (2004). [arXiv:math.DG/0405068]. (Cited on page 72.)

[123] Graham, C.R. and Witten, E., "Conformal anomaly of submanifold observables in AdS/CFT correspondence", Nucl. Phys. B, 546, 52-64, (1999). [DOI], [arXiv:hep-th/9901021]. (Cited on page 70 .)

[124] Gubser, S.S., "AdS/CFT and gravity", Phys. Rev. D, 63, 084017, (2001). [DOI], [arXiv:hepth/9912001 [hep-th]]. (Cited on page 76.)

[125] Gubser, S.S., Klebanov, I.R. and Polyakov, A.M., "Gauge theory correlators from non-critical string theory", Phys. Lett. B, 428, 105-114, (1998). [DOI], [arXiv:hep-th/9802109 [hep-th]]. (Cited on page 68.)

[126] Hartle, J.B. and Hawking, S.W., "Path-integral derivation of black hole radiance", Phys. Rev. D, 13, 2188-2203, (1976). [DOI]. (Cited on pages 19 and 20.)

[127] Hartle, J.B. and Hawking, S.W., "Wave Function of the Universe", Phys. Rev. D, 28, 29602975, (1983). [DOI]. (Cited on page 19.)

[128] Hawking, S.W., "Particle creation by black holes", Commun. Math. Phys., 43, 199-220, (1975). [DOI]. (Cited on page 5.) 
[129] Hawking, S.W., "Breakdown of Predictability in Gravitational Collapse", Phys. Rev. D, 14, 2460-2473, (1976). [DOI]. (Cited on page 80.)

[130] Hawking, S., Maldacena, J.M. and Strominger, A., "DeSitter entropy, quantum entanglement and ADS/CFT", J. High Energy Phys., 2001(05), 001, (2001). [DOI], [arXiv:hep-th/0002145 [hep-th]]. (Cited on pages 69,75 , and 76 .)

[131] Henningson, M. and Skenderis, K., "The Holographic Weyl anomaly", J. High Energy Phys., 1998(07), 023, (1998). [DOI], [arXiv:hep-th/9806087 [hep-th]]. (Cited on pages 63, 65, 71, and 72.)

[132] Ho, J. and Kang, G., "Superradiance and the statistical mechanical entropy of rotating BTZ black holes", Phys. Lett. B, 445, 27-35, (1998). [DOI], [arXiv:gr-qc/9806118 [gr-qc]]. (Cited on page 45.)

[133] Holzhey, C., Larsen, F. and Wilczek, F., "Geometric and renormalized entropy in conformal field theory", Nucl. Phys. B, 424, 443-467, (1994). [DOI], [arXiv:hep-th/9403108]. (Cited on page 9.)

[134] Hung, L.-Y., Myers, R.C. and Smolkin, M., "On Holographic Entanglement Entropy and Higher Curvature Gravity", J. High Energy Phys., 2011(4), 025, (2011). [DOI], [arXiv:1101.5813 [hep-th]]. (Cited on pages 20 and 65.)

[135] Iellici, D. and Moreira Jr, E.S., "Ambiguity in the evaluation of effective action on the cone", Phys. Rev. D, 60, 124015, (1999). [DOI], [arXiv:hep-th/9807184 [hep-th]]. (Cited on page 28.)

[136] Iellici, D. and Moretti, V., "Thermal partition function of photons and gravitons in a Rindler wedge", Phys. Rev. D, 54, 7459-7469, (1996). [DOI], [arXiv:hep-th/9607015 [hep-th]]. (Cited on page 49.)

[137] Iellici, D. and Moretti, V., "Kabat's Surface Terms in the Zeta-Function Approach", in Bassan, M., Ferrari, V., Francaviglia, M., Fucito, F. and Modena, I., eds., General Relativity and Gravitational Physics, Proceedings of the 12th Italian Conference, Rome, Italy, September 23-27, 1996, pp. 317-321, (World Scientific, Singapore; River Edge, NJ, 1997). [arXiv:hepth/9703088]. (Cited on page 28.)

[138] Israel, W., "Thermo-field dynamics of black holes", Phys. Lett. A, 57, 107-110, (1976). [DOI]. (Cited on page 21.)

[139] Iwashita, Y., Kobayashi, T., Shiromizu, T. and Yoshino, H., "Holographic entanglement entropy of de Sitter braneworld", Phys. Rev. D, 74, 064027, (2006). [DOI], [arXiv:hep-th/0606027 [hep-th]]. (Cited on pages 69 and 70.)

[140] Iyer, V. and Wald, R.M., "Comparison of Noether charge and Euclidean methods for computing the entropy of stationary black holes", Phys. Rev. D, 52, 4430-4439, (1995). [DOI], [arXiv:gr-qc/9503052 [gr-qc]]. (Cited on page 32.)

[141] Jacobson, T., "Black Hole Entropy and Induced Gravity", arXiv, e-print, (1994). [arXiv:grqc/9404039]. (Cited on pages 5, 33, and 74.)

[142] Jacobson, T., "A Note on Hartle-Hawking vacua", Phys. Rev. D, 50, 6031-6032, (1994). [DOI], [arXiv:gr-qc/9407022 [gr-qc]]. (Cited on page 21.)

[143] Jacobson, T.A., "Renormalization and black hole entropy in Loop Quantum Gravity", Class. Quantum Grav., 24, 4875-4879, (2007). [DOI], [arXiv:0707.4026 [gr-qc]]. (Cited on page 79.) 
[144] Jacobson, T., Kang, G. and Myers, R.C., "On black hole entropy", Phys. Rev. D, 49, 65876598, (1994). [DOI], [arXiv:gr-qc/9312023]. (Cited on pages 25 and 32.)

[145] Jacobson, T. and Myers, R.C., "Black hole entropy and higher curvature interactions", Phys. Rev. Lett., 70, 3684-3687, (1993). [DOI], [arXiv:hep-th/9305016]. (Cited on page 23.)

[146] Jacobson, T. and Parentani, R., "Horizon Entropy", Found. Phys., 33, 323-348, (2003). [DOI], [arXiv:gr-qc/0302099]. (Cited on page 6.)

[147] Jacobson, T. and Parentani, R., "Black hole entanglement entropy regularized in a freely falling frame", Phys. Rev. D, 76, 024006, (2007). [DOI], [arXiv:hep-th/0703233]. (Cited on page 80.)

[148] Jing, J. and Yan, M.-L., "Entropies of the general nonextreme stationary axisymmetric black hole: Statistical mechanics and thermodynamics", Phys. Rev. D, 61, 044016, (2000). [DOI], [arXiv:gr-qc/9907011 [gr-qc]]. (Cited on page 45.)

[149] Jing, J. and Yan, M.-L., "Quantum entropy of the Kerr black hole arising from gravitational perturbation", Phys. Rev. D, 64, 064015, (2001). [DOI], [arXiv:gr-qc/0104054 [gr-qc]]. (Cited on page 45.)

[150] Kabat, D.N., "Black hole entropy and entropy of entanglement", Nucl. Phys. B, 453, 281302, (1995). [DOI], [arXiv:hep-th/9503016]. (Cited on page 35.)

[151] Kabat, D.N., Shenker, S.H. and Strassler, M.J., "Black hole entropy in the O(N) model", Phys. Rev. D, 52, 7027-7036, (1995). [DOI], [arXiv:hep-th/9506182]. (Cited on pages 39 and 78.)

[152] Kabat, D.N. and Strassler, M.J., "A Comment on entropy and area", Phys. Lett. B, 329, 46-52, (1994). [DOI], [arXiv:hep-th/9401125]. (Cited on pages 21 and 25.)

[153] Kenmoku, M., Ishimoto, K., Nandi, Kamal Kanti and Shigemoto, K., "Scalar field contribution to rotating black hole entropy", Phys. Rev. D, 73, 064004, (2006). [DOI], [arXiv:grqc/0510012 [gr-qc]]. (Cited on page 45.)

[154] Kim, W., Kim, Y.-W. and Park, Y.-J., "Entropy of $2+1$ de Sitter space with the GUP", J. Korean Phys. Soc., 49, 1360-1364, (2006). [arXiv:gr-qc/0604065 [gr-qc]]. (Cited on page 80.)

[155] Kim, W., Park, Y.-J., Son, E.J. and Yoon, M.S., "Statistical Entropy and Superradiance in 2+1 Dimensional Acoustic Black Holes", J. Korean Phys. Soc., 49, 15-20, (2006). [DOI], [arXiv:gr-qc/0504127 [gr-qc]]. (Cited on page 45.)

[156] Kim, Y.-W. and Park, Y.-J., "Entropy of the Schwarzschild black hole to all orders in the Planck length", Phys. Lett. B, 655, 172-177, (2007). [DOI], [arXiv:0707.2128 [gr-qc]]. (Cited on page 80.)

[157] Klebanov, I.R., Kutasov, D. and Murugan, A., "Entanglement as a Probe of Confinement", Nucl. Phys. B, 796, 274-293, (2008). [DOI], [arXiv:0709.2140 [hep-th]]. (Cited on page 69.)

[158] Koksma, J.F., Prokopec, T. and Schmidt, M.G., "Entropy and correlators in quantum field theory", Ann. Phys. (N.Y.), 325, 1277-1303, (2010). [DOI], [arXiv:1002.0749 [hep-th]]. (Cited on page 80.)

[159] Koksma, J.F., Prokopec, T. and Schmidt, M.G., "Decoherence and Dynamical Entropy Generation in Quantum Field Theory", arXiv, e-print, (2011). [arXiv:1101.5323 [quant-ph]]. (Cited on page 80.) 
[160] Koksma, J.F., Prokopec, T. and Schmidt, M.G., "Decoherence in an Interacting Quantum Field Theory: Thermal Case", Phys. Rev. D, 83, 085011, (2011). [DOI], [arXiv:1102.4713 [hep-th]]. (Cited on page 80.)

[161] Korepin, V.E., "Universality of Entropy Scaling in One Dimensional Gapless Models", Phys. Rev. Lett., 92, 096402, (2004). [DOI]. (Cited on page 9.)

[162] Larsen, F. and Wilczek, F., "Geometric Entropy, Wave Functionals, and Fermions", Ann. Phys. (N.Y.), 243, 280-298, (1995). [DOI], [arXiv:hep-th/9408089]. (Cited on page 35.)

[163] Larsen, F. and Wilczek, F., "Renormalization of black hole entropy and of the gravitational coupling constant", Nucl. Phys. B, 458, 249-266, (1996). [DOI], [arXiv:hep-th/9506066]. (Cited on pages 35 and 37.)

[164] Latorre, J.I., Lütken, C.A., Rico, E. and Vidal, G., "Fine-grained entanglement loss along renormalization-group flows", Phys. Rev. A, 71, 034301, (2005). [DOI], [arXiv:quantph/0404120 [quant-ph]]. (Cited on page 80.)

[165] Livine, E.R. and Terno, D.R., "Quantum black holes: Entropy and entanglement on the horizon", Nucl. Phys. B, 741, 131-161, (2006). [DOI], [arXiv:gr-qc/0508085]. (Cited on page 79.)

[166] Lovelock, D., "The Einstein tensor and its generalizations", J. Math. Phys., 12, 498-501, (1971). [DOI]. (Cited on page 22.)

[167] Maldacena, J.M., "The Large N Limit of Superconformal Field Theories and Supergravity", Adv. Theor. Math. Phys., 2, 231-252, (1998). [arXiv:hep-th/9711200 [hep-th]]. (Cited on pages 63, 68, and 76.)

[168] Maldacena, J.M., "Eternal black holes in anti-de Sitter", J. High Energy Phys., 2003(04), 021, (2003). [DOI], [arXiv:hep-th/0106112]. (Cited on page 78.)

[169] Mandal, G., Sengupta, A.M. and Wadia, S.R., "Classical solutions of 2-dimensional string theory", Mod. Phys. Lett. A, 6, 1685-1692, (1991). [DOI]. (Cited on page 51.)

[170] Mann, R.B. and Solodukhin, S.N., "Conical geometry and quantum entropy of a charged Kerr black hole", Phys. Rev. D, 54, 3932-3940, (1996). [DOI], [arXiv:hep-th/9604118]. (Cited on pages $28,29,30,31$, and 33.)

[171] Mann, R.B. and Solodukhin, S.N., "Quantum scalar field on three-dimensional (BTZ) black hole instanton: Heat kernel, effective action and thermodynamics", Phys. Rev. D, 55, 36223632, (1997). [DOI], [arXiv:hep-th/9609085]. (Cited on pages 54 and 55.)

[172] Mann, R.B. and Solodukhin, S.N., "Universality of quantum entropy for extreme black holes", Nucl. Phys. B, 523, 293-307, (1998). [DOI], [arXiv:hep-th/9709064]. (Cited on pages 14, 55, 57, and 58.)

[173] Mann, R.B., Tarasov, L. and Zelnikov, A., "Brick walls for black holes", Class. Quantum Grav., 9, 1487-1494, (1992). [DOI]. (Cited on page 44.)

[174] McKean Jr, H.P. and Singer, I.M., "Curvature and the eigenvalues of the Laplacian", J. Differ. Geom., 1(1-2), 43-69, (1967). (Cited on page 24.)

[175] Metlitski, M.A., Fuertes, C.A. and Sachdev, S., "Entanglement Entropy in the $O(N)$ model", Phys. Rev. B, 80, 115122, (2009). [DOI], [arXiv:0904.4477 [cond-mat.stat-mech]]. (Cited on page 39.) 
[176] Moretti, V., "Geometric entropy and curvature coupling in conical spaces: Zeta function approach", Class. Quantum Grav., 14, L123-L127, (1997). [DOI], [arXiv:hep-th/9701099 [hepth]]. (Cited on page 28.)

[177] Moretti, V. and Iellici, D., "Optical approach for the thermal partition function of photons", Phys. Rev. D, 55, 3552-3563, (1997). [DOI], [arXiv:hep-th/9610180 [hep-th]]. (Cited on page 49.)

[178] Mukohyama, S. and Israel, W., "Black holes, brick walls and the Boulware state", Phys. Rev. D, 58, 104005, (1998). [DOI], [arXiv:gr-qc/9806012 [gr-qc]]. (Cited on page 44.)

[179] Myers, R.C., "Black hole entropy in two-dimensions", Phys. Rev. D, 50, 6412-6421, (1994). [DOI], [arXiv:hep-th/9405162 [hep-th]]. (Cited on page 50.)

[180] Myers, R.C. and Perry, M.J., "Black Holes in Higher Dimensional Space-Times", Ann. Phys. (N.Y.), 172, 304, (1986). [DOI]. (Cited on pages 60 and 65.)

[181] Myers, R.C. and Sinha, A., "Holographic c-theorems in arbitrary dimensions", J. High Energy Phys., 2011(01), 125, (2011). [DOI], [arXiv:1011.5819 [hep-th]]. (Cited on pages 24 and 65.)

[182] Nelson, B.L. and Panangaden, P., "Scaling behavior of interacting quantum fields in curved space-time", Phys. Rev. D, 25, 1019-1027, (1982). [DOI]. (Cited on page 39.)

[183] Nesterov, D. and Solodukhin, S.N., "Short-distance regularity of Green's function and UV divergences in entanglement entropy", J. High Energy Phys., 2010(09), 041, (2010). [DOI], [arXiv:1008.0777 [hep-th]]. (Cited on pages 14, 15, and 80.)

[184] Nesterov, D. and Solodukhin, S.N., "Gravitational effective action and entanglement entropy in UV modified theories with and without Lorentz symmetry", Nucl. Phys. B, 842, 141-171, (2011). [DOI], [arXiv:1007.1246 [hep-th]]. (Cited on pages 14, 15, 16, 20, 33, 34, and 80.)

[185] Nishioka, T., Ryu, S. and Takayanagi, Tadashi, "Holographic entanglement entropy: an overview", J. Phys. A: Math. Theor., 42, 504008, (2009). [DOI], [arXiv:0905.0932 [hep-th]]. (Cited on pages 6 and 68.)

[186] Padmanabhan, T., "Finite entanglement entropy from the zero-point-area of spacetime", Phys. Rev. D, 82, 124025, (2010). [DOI], [arXiv:1007.5066 [gr-qc]]. (Cited on page 80.)

[187] Randall, L. and Sundrum, R., "An alternative to compactification", Phys. Rev. Lett., 83, 4690-4693, (1999). [DOI], [arXiv:hep-th/9906064 [hep-th]]. (Cited on page 76.)

[188] Ryu, S. and Takayanagi, T., "Aspects of Holographic Entanglement Entropy", J. High Energy Phys., 2006(08), 045, (2006). [DOI], [arXiv:hep-th/0605073 [hep-th]]. (Cited on pages 62 and 68.)

[189] Ryu, S. and Takayanagi, T., "Holographic derivation of entanglement entropy from AdS/CFT", Phys. Rev. Lett., 96, 181602, (2006). [DOI], [arXiv:hep-th/0603001 [hep-th]]. (Cited on pages 6 and 68.)

[190] Sakharov, A.D., "Vacuum quantum fluctuations in curved space and the theory of gravitation", Sov. Phys. Dokl., 12, 1040-1041, (1968). (Cited on page 74.)

[191] Schwimmer, A. and Theisen, S., "Entanglement Entropy, Trace Anomalies and Holography", Nucl. Phys. B, 801, 1-24, (2008). [DOI], [arXiv:0802.1017 [hep-th]]. (Cited on page 70.) 
[192] Sen, A., "State Operator Correspondence and Entanglement in $\mathrm{AdS}_{2} / \mathrm{CFT}_{1}$ ", arXiv, e-print, (2011). [arXiv:1101.4254 [hep-th]]. (Cited on page 78.)

[193] Skenderis, K., "Asymptotically Anti-de Sitter space-times and their stress energy tensor", Int. J. Mod. Phys. A, 16, 740-749, (2001). [DOI], [arXiv:hep-th/0010138 [hep-th]]. (Cited on page 68.)

[194] Skenderis, K. and Solodukhin, S.N., "Quantum effective action from the AdS/CFT correspondence", Phys. Lett. B, 472, 316-322, (2000). [DOI], [arXiv:hep-th/9910023 [hep-th]]. (Cited on pages 69 and 70.)

[195] Sokolov, D.D. and Starobinsky, A.A., "The structure of the curvature tensor at conical singularities", Sov. Phys. Dokl., 22, 312-313, (1977). (Cited on page 21.)

[196] Solodukhin, S.N., "The conical singularity and quantum corrections to entropy of black hole", Phys. Rev. D, 51, 609-617, (1995). [DOI], [arXiv:hep-th/9407001]. (Cited on pages 5, 25, 27, $28,33,50$, and 62.)

[197] Solodukhin, S.N., 'On 'Nongeometric' contribution to the entropy of black hole due to quantum corrections", Phys. Rev. D, 51, 618-621, (1995). [DOI], [arXiv:hep-th/9408068]. (Cited on pages 5, 26, 27, and 28.)

[198] Solodukhin, S.N., "One loop renormalization of black hole entropy due to nonminimally coupled matter", Phys. Rev. D, 52, 7046-7052, (1995). [DOI], [arXiv:hep-th/9504022]. (Cited on pages 37 and 40.)

[199] Solodukhin, S.N., "Black hole entropy: statistical mechanics agrees thermodynamics", Phys. Rev. D, 54, 3900-3903, (1996). [DOI], [arXiv:hep-th/9601154]. (Cited on pages 41, 49, and 51.)

[200] Solodukhin, S.N., "Non-minimal coupling and quantum entropy of black hole", Phys. Rev. D, 56, 4968-4974, (1997). [DOI], [arXiv:hep-th/9612061]. (Cited on page 45.)

[201] Solodukhin, S.N., "Entropy of Schwarzschild black hole and string-black hole correspondence", Phys. Rev. D, 57, 2410-2414, (1998). [DOI], [arXiv:hep-th/9701106]. (Cited on page 66.)

[202] Solodukhin, S.N., "Entanglement entropy of black holes and AdS/CFT correspondence", Phys. Rev. Lett., 97, 201601, (2006). [DOI], [arXiv:hep-th/0606205 [hep-th]]. (Cited on pages $69,70,73$, and 76.$)$

[203] Solodukhin, S.N., "Entanglement entropy and the Ricci flow", Phys. Lett. B, 646, 268-274, (2007). [DOI], [arXiv:hep-th/0609045]. (Cited on page 80.)

[204] Solodukhin, S.N., "Entanglement entropy, conformal invariance and extrinsic geometry", Phys. Lett. B, 665, 305-309, (2008). [DOI], [arXiv:0802.3117 [hep-th]]. (Cited on pages 16, 20, and 63.)

[205] Solodukhin, S.N., "Entanglement Entropy in Non-Relativistic Field Theories", J. High Energy Phys., 2010(04), 101, (2010). [DOI], [arXiv:0909.0277 [hep-th]]. (Cited on pages 16 and 39.)

[206] Solodukhin, S.N., "Entanglement entropy of round spheres", Phys. Lett. B, 693, 605-608, (2010). [DOI], [arXiv:1008.4314 [hep-th]]. (Cited on pages 57 and 59.)

[207] Sommerfeld, A., "Über verzweigte Potentiale im Raum", Proc. London Math. Soc. (1), XXVIII, 395-429, (1897). [DOI]. (Cited on page 11.) 
[208] Srednicki, M., "Entropy and area", Phys. Rev. Lett., 71, 666-669, (1993). [DOI], [arXiv:hepth/9303048]. (Cited on pages 5 and 9.)

[209] Strominger, A. and Vafa, C., "Microscopic Origin of the Bekenstein-Hawking Entropy", Phys. Lett. B, 379, 99-104, (1996). [DOI], [arXiv:hep-th/9601029]. (Cited on page 6.)

[210] Sun, X. and Liu, W., "Improved black hole entropy calculation without cutoff", Mod. Phys. Lett. A, 19, 677-680, (2004). [DOI]. (Cited on page 80.)

[211] Susskind, L., "Some Speculations about Black Hole Entropy in String Theory", arXiv, eprint, (1993). [arXiv:hep-th/9309145]. (Cited on page 5.)

[212] Susskind, L., "The world as a hologram", J. Math. Phys., 36, 6377-6396, (1995). [DOI], [arXiv:hep-th/9409089 [hep-th]]. (Cited on page 68.)

[213] Susskind, L. and Uglum, J., "Black hole entropy in canonical quantum gravity and superstring theory", Phys. Rev. D, 50, 2700-2711, (1994). [DOI], [arXiv:hep-th/9401070]. (Cited on pages $5,33,44$, and 78.)

[214] 't Hooft, G., "On the Quantum Structure of a Black Hole", Nucl. Phys. B, 256, 727-745, (1985). [DOI]. (Cited on pages 5, 41, 44, and 51.)

[215] 't Hooft, G., "Dimensional Reduction in Quantum Gravity", arXiv, e-print, (1993). [arXiv:grqc/9310026]. (Cited on page 68.)

[216] 't Hooft, G., "Quantum gravity without space-time singularities or horizons", arXiv, e-print, (2009). [arXiv:0909.3426 [gr-qc]]. (Cited on page 66.)

[217] 't Hooft, G., "The Conformal Constraint in Canonical Quantum Gravity", arXiv, e-print, (2010). [arXiv:1011.0061 [gr-qc]]. (Cited on page 66.)

[218] 't Hooft, G., "Probing the small distance structure of canonical quantum gravity using the conformal group", arXiv, e-print, (2010). [arXiv:1009.0669 [gr-qc]]. (Cited on page 66.)

[219] Vassilevich, D.V., "Heat kernel expansion: User's manual", Phys. Rep., 388, 279-360, (2003). [DOI], [arXiv:hep-th/0306138 [hep-th]]. (Cited on pages 24, 58, and 61.)

[220] Visser, M., "Sakharov's induced gravity: A modern perspective", Mod. Phys. Lett. A, 17, 977-992, (2002). [DOI], [arXiv:gr-qc/0204062]. (Cited on page 74.)

[221] Wald, R.M., "Black hole entropy is the Noether charge", Phys. Rev. D, 48, R3427-R3431, (1993). [DOI], [arXiv:gr-qc/9307038]. (Cited on pages 25, 32, and 50.)

[222] Witten, E., "On string theory and black holes", Phys. Rev. D, 44, 314-324, (1991). [DOI]. (Cited on page 51.)

[223] Witten, E., "Anti-de Sitter space and holography", Adv. Theor. Math. Phys., 2, 253-291, (1998). [arXiv:hep-th/9802150 [hep-th]]. (Cited on page 68.)

[224] Wu, S.-Q. and Yan, M.-L., "Entropy of Kerr-de Sitter black hole due to arbitrary spin fields", Phys. Rev. D, 69, 044019, (2004). [DOI], [arXiv:gr-qc/0303076 [gr-qc]]. (Cited on page 45.)

[225] Yoon, M., Ha, J. and Kim, W., "Entropy of Reissner-Nordstrom black holes with minimal length revisited", Phys. Rev. D, 76, 047501, (2007). [DOI], [arXiv:0706.0364 [gr-qc]]. (Cited on page 80.) 
[226] Zaslavsky, O.B., "Geometry of nonextreme black holes near the extreme state", Phys. Rev. $D, \mathbf{5 6}, 2188-2191,(1997)$. [DOI], [arXiv:gr-qc/9707015 [gr-qc]]. (Cited on page 55.)

[227] Zerbini, S., Cognola, G. and Vanzo, L., "Euclidean approach to the entropy for a scalar field in Rindler-like space-times", Phys. Rev. D, 54, 2699-2710, (1996). [DOI], [arXiv:hep-th/9603106 [hep-th]]. (Cited on page 28.) 